\title{
A PHENOMENOLOGICAL STUDY: AN ANALYSIS OF NO CONFIDENCE VOTES AMONG PRESIDENTS IN CALIFORNIA COMMUNITY COLLEGES
}

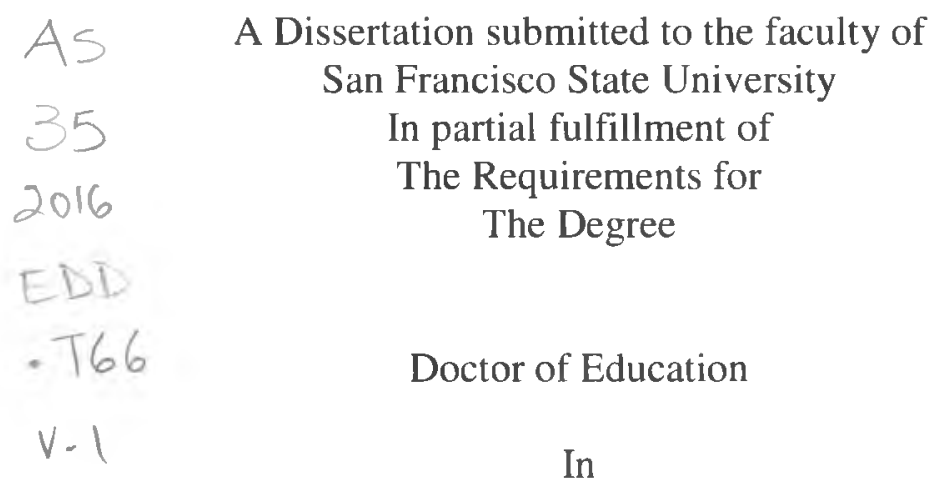

Educational Leadership

Lisa Ann-Marie Thomas

San Francisco, California

December 2015 


\section{Copyright by}

Lisa Ann-Marie Thomas

2015 


\section{CERTIFICATION OF APPROVAL}

I certify that I have read $A$ Phenomenological Study: An Analysis of No Confidence Votes Among Presidents in California Community Colleges by Lisa Thomas, and that in my opinion this work meets the criteria for approving a dissertation submitted in partial fulfillment of the requirements for the degree: Doctor of Education in Educational Leadership at San Francisco State University.
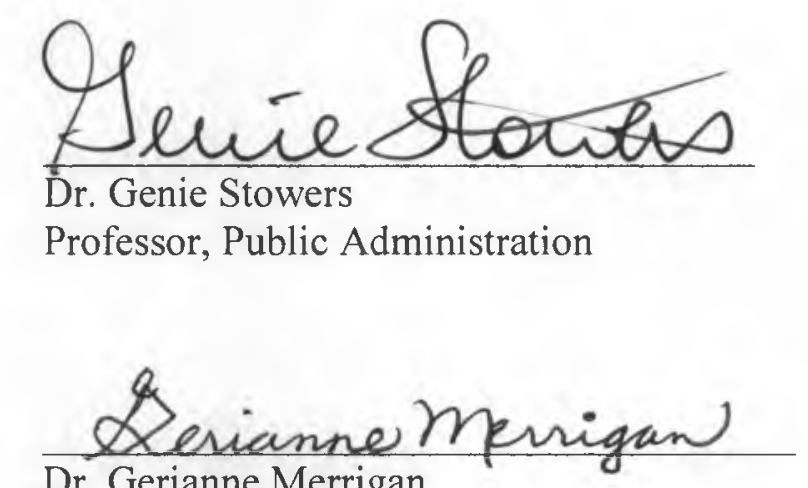

Dr. Gerianne Merrigan

Professor, Communication Studies

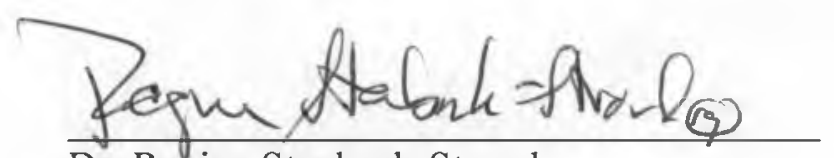

Dr. Regina Stanback-Stroud

President/ CEO, Skyline College 


\title{
A PHENOMENOLOGICAL STUDY: AN ANALYSIS OF NO CONFIDENCE VOTES AMONG PRESIDENTS IN CALIFORNIA COMMUNITY COLLEGES
}

\author{
Lisa Thomas \\ San Francisco State University \\ 2015
}

\begin{abstract}
This phenomenological study explored the growing concerns, meanings, and implications of no confidence vote sanctions against presidents in California's community colleges. Using in-depth interviews, this study attempted to contribute in-depth knowledge about this ubiquitous phenomenon and to profoundly expose the potential implications of the vote on the context of leadership in community colleges through the constructed narratives of presidents. Weick's (1995) sensemaking theory and analytical framework was the key approach used for a thematic narrative analysis of how leaders made sense of this phenomenon. The study's findings identified the fundamental gaps between the sensemaking of leaders as opposed to the sponsors of the vote, as well as the personal and professional repercussions of the vote on these leaders and their careers. The results of this study have important implications for aspiring and current leaders, policy makers, as well as other leaders in California's community colleges.
\end{abstract}

I certify that the Abstract is a correct representation of the content of this dissertation.

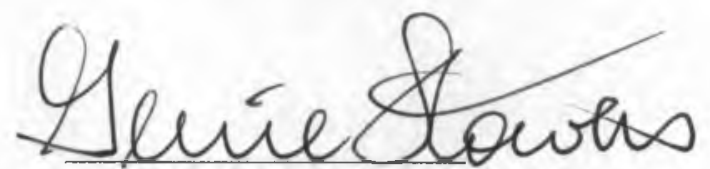

Chair, Dissertation Committee

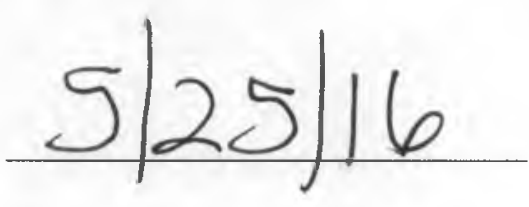

Date 


\section{ACKNOWLEDGEMENTS}

First, I want to boldly thank God, Jesus Christ, for never letting me go and being with me throughout this entire process and my life. He asked me to hold on to Philippians 1:6, and I did and thank Him for everything.

I want to acknowledge and thank my dissertation chair and most patient supporter, Dr. Genie Stowers, whom, without her agreeing to go on this arduous journey with me, this study would not have happened. I also want to thank Dr. Gerianne Merrigan and Dr. Regina Stanback-Stroud who are two of the most knowledgeable and amazing powerhouses behind this study and I can only aspire to be like you one day. I would also like to thank the program director, Dr. Robert Gabriner, his staff, and the entire faculty of the Educational Leadership Program at San Francisco State University, your investment in me was not and is not wasted.

Last but most certainly not least, I emphatically want to thank my family and friends. To Rachel Hasbun, thank you for holding my hands through this entire journey and for your support, prayers, compassion and complete devotion along the way. To my mother, Rev.

Ileen Thomas; grandmother, Ms. Pearlyn Green; Jessica Sharp; Dr. Rose Myers, and countless other giants of faith, prayer warriors, counselors, professional colleagues, editors, family and friends, thank you! I really know who I can rely on to go with me and do battle and I love you all for being there for me. You all rock! 


\section{DEDICATION}

I dedicate this research project to the four very brave participants in this study: Susan, Debbie, Paul, and Bill (pseudonyms). Without you agreeing to share your stories about your upbringing and growing pains as youths, personal and family lives, and courage to lead during times of great uncertainty and at a great cost, this study would not exist. I will never be able to thank and praise you enough. Thank you for allowing me to peer into the window of your worlds and to capture snippets of your lives and experiences. My deepest desire in sharing your stories is that, and I need to say it in a colloquial way, I hope I done right by you. May God bless each of you! 


\section{TABLE OF CONTENTS}

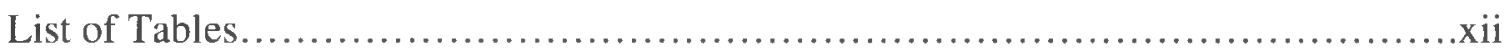

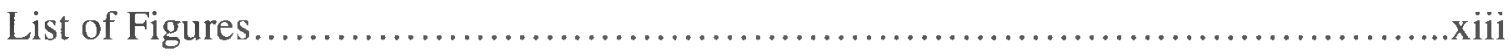

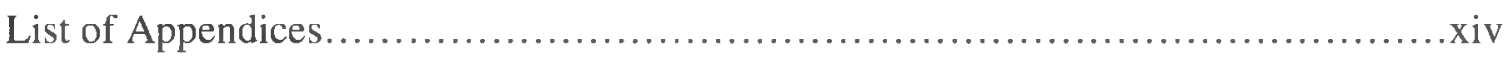

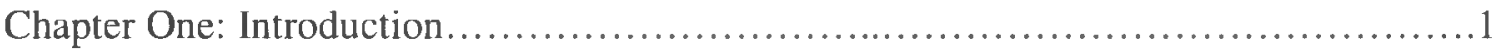

Definition of No Confidence Vote and Crises.........................................8

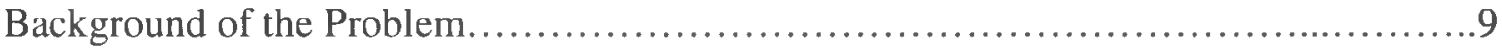

Nationwide no confidence vote data..................................... 12

Leadership challenges in higher education.................................23

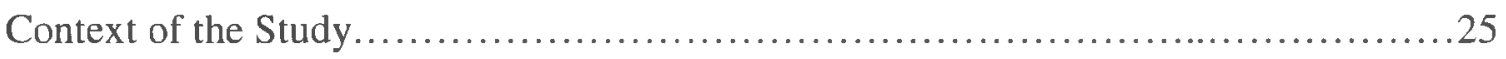

Leadership challenges in California's Community Colleges.....................27

California Community Colleges no confidence vote data......................29

Description of the Problem...........................................................

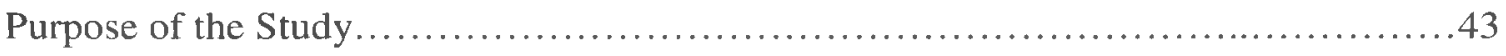

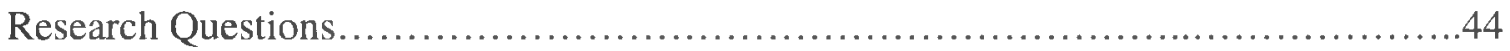

Statement of Ethical Considerations.............................................45

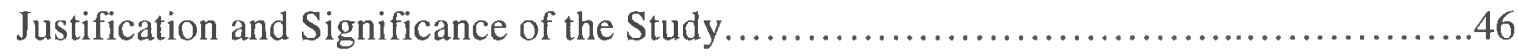

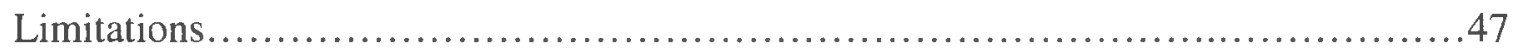

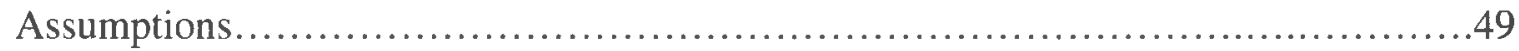

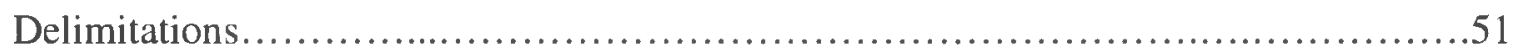




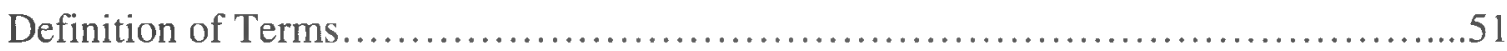

Chapter Two: Introduction to Literature Search Methods............................54

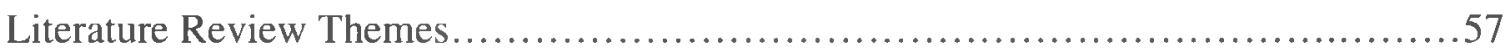

Theme One: Leadership performance and challenges associated with NCVs.....76

Theme Two: Contextual Factors Contributing To the Vote.....................111

Theme Three: Communication Factors.................................. 128

Theme Four: Other Implications and Consequences of NCVs...................136

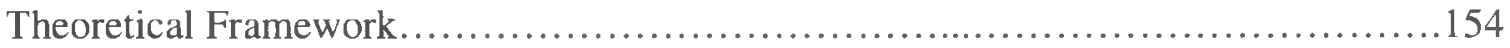

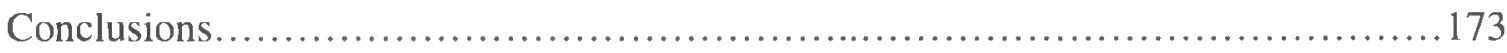

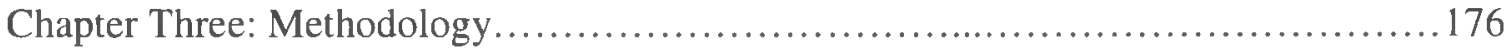

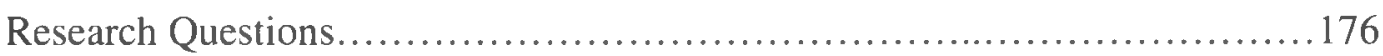

Research Approach.................................................. 177

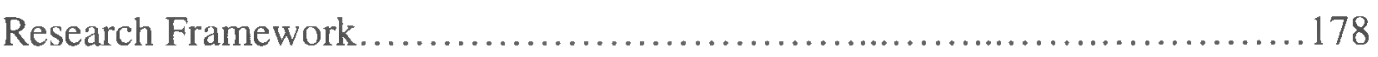

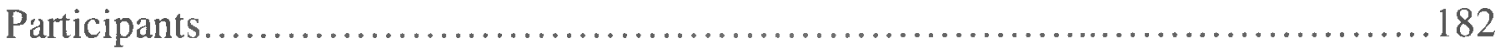

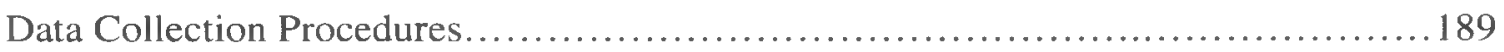

Data Analysis Procedures................................................ 192

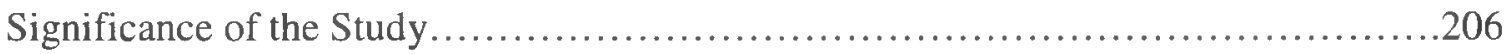

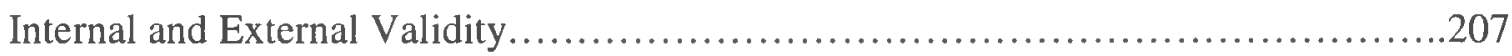

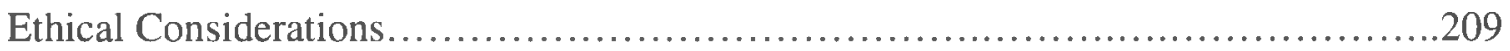

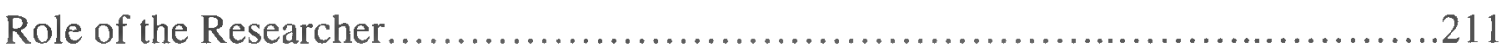

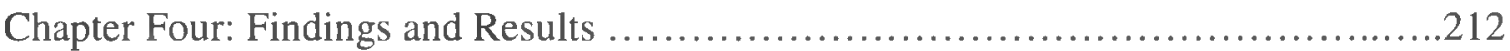




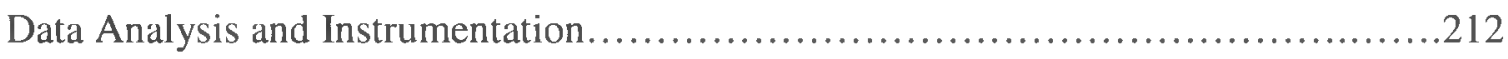

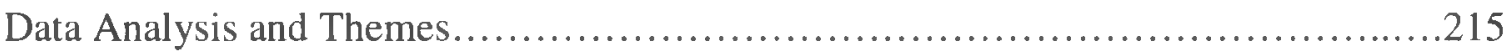

Demographical and Background Information......................................2219

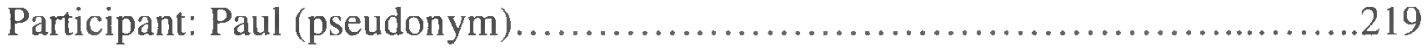

Participant: Bill (pseudonym)............................................222

Participant: Debbie (Pseudonym)...........................................231

Participant: Susan (pseudonym)..........................................2239

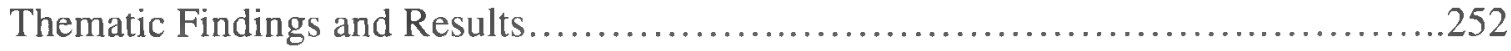

Theme One: Known Triggers of NCV .....................................254

Theme Two: Crucial Blind Spots in NCV ................................28

Theme Three: Implications of NCV ...................................299

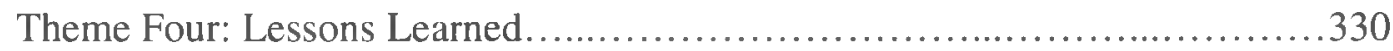

Conclusion and Summary of Results.............................................. 336

Chapter Five: Discussion, Conclusions and Recommendations......................340

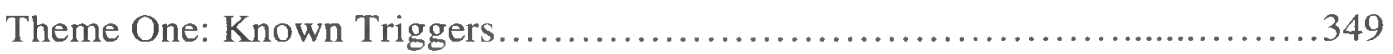

Theme Two: Crucial blind spots.............................................367

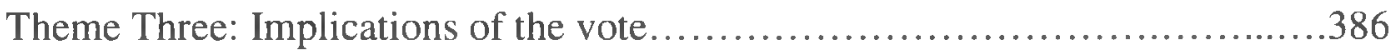

Theme Four. Lessons learned............................................414

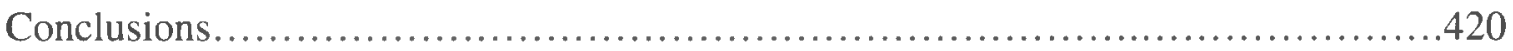

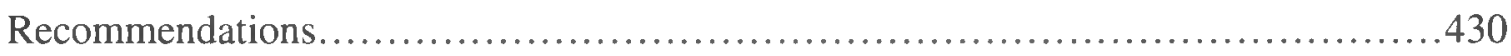

Further Research.................................................................. 


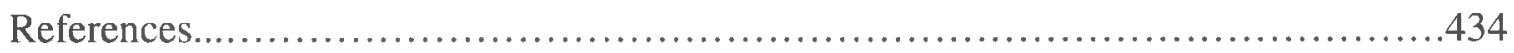

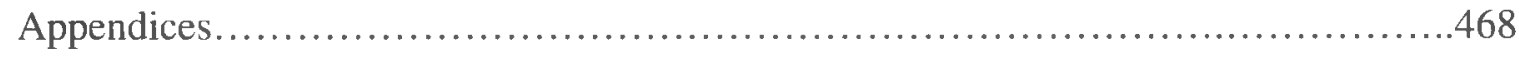




\section{LIST OF TABLES}

Table

Table 1. Rationales for NCV Cases from Sample... 16

Table 2. Polarized Arguments about the Meanings of NCV in Higher Education.........18

Table 3. NCV Reported to California Community Colleges............................ 35

Table 4. Potential Costs Associated with the Unexpected Ousting of Leaders...........71

Table 5. Shared Governance Law- AB 1725 for California CC.................... 116

Table 6. Votes of No Confidence for Each Study Participant......................... 187

Table 7. Methods: Bogdan and Biklen's (2007) Combined with Creswell and Plano

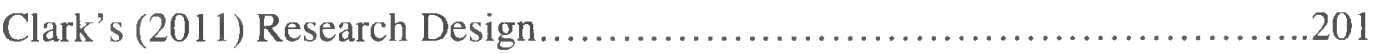

Table 8. Six Steps for Coding and Thematic Analysis Process.......................201

Table 9. Comparisons between the Researcher and NVIVO 10's Themes...............205

Table 10. Intersections between Research and Protocol Questions .............................213

Table 11. Overview of Dataset................................................. 15

Table 12. Significant Cross-Cutting Themes across Study Participants................215

Table 13. Cycle 2: Research Themes and Subthemes.............................253 


\section{LIST OF FIGURES}

Figures

Page

Figure 1. Nationwide Sample of NCVs Among Presidents in Higher Ed. by years........13

Figure $2 . \mathrm{NCVs}$ by gender....................................................... 14

Figure 3. Number of NCVs from 1989 to Present................................. 14

Figure 4. NCVs in California Community College 1990 - Present......................30

Figure 5. California CC NCV Cases by gender from 1990 to present...................30

Figure 6. California CC NCV Cases Reported in the Media from 1990 to Present........30

Figure 7. Contributing Factors to NCVs in California CC from 1993-2003..............36

Figure 8. Number of NCV by Gender.............................................

Figure 9. Conceptual Map of Weick's (1995) sensemaking approach.................. 156

Figure 10. Data Research Themes and Subthemes...............................253

Figure 11. Leader's Sensemaking: Double Blind-Spots in NCV Crisis.................382 


\section{APPENDICES}

Appendix $\quad$ Page

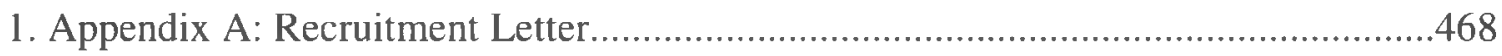

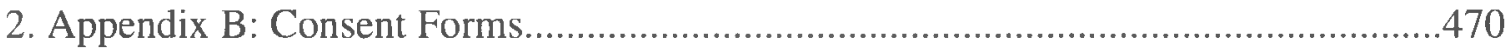

3. Appendix C: Interview Protocol............................................473

3. Appendix D: San Francisco State University Dissertation Permission Letter.........476

4. Appendix E: No Confidence Votes in California Community Colleges...................477

5. Appendix F: Data Conversion Table for Study Participants...................................482

6. Appendix G: Theme Four: Lessons Learned from Participants......................483

7. Appendix H: IRB Completion Form.................................... 485

8. Appendix I: NIH Form................................................ 486 


\section{Chapter One}

\section{Introduction}

Members of Harvard's Faculty of Arts and Sciences passed a vote of no confidence yesterday in Lawrence H. Summers, dealing a stunning rebuke to the president of one of the world's top universities...The vote, which astonished even some fierce critics, made clear that the faculty's disenchantment with Summers is deeper than many had imagined. After the vote, Summers renewed his pledge to improve relations with Harvard's scholars. (Bombardieri \& Abel, 2005)

In higher education, there has been a proliferation of reports about university and college presidents in crisis, like Lawrence Summers, whose leadership tenures were under a threat of, or received, a no confidence vote from within their institutions. A no confidence vote (NCV) is defined as a "vote in which members of a group are asked to indicate that they do not support the person or group in power" (collinsdictionary.com, 2015). As a matter of fact, several authors have raised concerns about the growing usage (Kiley, 2013; Petrick, 2007), meanings (Schmidt, 2009; MacTaggart, 2012), and outcomes (Tierney, 2007;

Smith, 2003) of no confidence votes against presidents or other administrators in higher education. For instance, MacTaggart (2012) asserted that a no confidence vote is a signal that the leader is "incapable of leading" and should be ousted from their institutions (para. 3). While other authors have agreed that these votes do act as a type of performance evaluation of the leader (Lieu, 2005), other scholars have 
argued that their main emphasis is on the grievances and tensions between the administration and the sponsors of the vote (Kiley, 2013; Kuykendall, 2010; Schmidt, 2009; Tierney, 2007). As a result, there is a knowledge gap about these no confidence votes that do appear to have different and significant meanings for both leaders and those who use it against these leaders.

In addition to the potential significance of these no confidence votes, it appears these votes can occur at any time, for any reason against the leadership, and can be sponsored by any group (Schmidt, 2009). Meaning, these types of votes can be sponsored by any entity within or outside an organization, such as the faculty, union, or board (Kuykendall, 2010; Tierney, 2007). Despite the plethora of critical issues facing higher education and its leaders, such as economical or fiscal challenges, these votes can also happen (Schmidt, 2009; Shulock, 2002). Furthermore, these no confidence votes can also happen in institutional environments that may be supportive or hostile towards the leader, and they may not be correlated with the leader's level of performance or performance evaluation (Smith, 2003). Although these types of votes do not necessarily present the overall performance or evaluation of these leaders in the academy, and little is known about the meanings and implications of these votes in general, they do appear to evoke widespread interest and questions in the field about why these votes are happening and what's going really going on in these institutions (Wright, 2007). 
A major barrier to understanding this phenomenon is the lack of data about these votes in higher education sectors, which were limited to and mostly reported in trade journals and newspapers, such as The Chronicle and Inside Higher Education, for example. Apart from these types of sources that share reports on these types of votes, the lack of knowledge is also predicated by the fact that no official agency was found that collected or analyzed national data. A single agency in California was found and they have analyzed self-reported data from community colleges about these votes. Perhaps, knowledge about these no confidence votes remains elusive because of limited data sources and little to no studies with analysis of these sanction are available. From these sources found, however, the main concerns about these no confidence votes were centered on their usage, proliferation, meanings and outcomes, which appears to be complex and convoluted with other challenges in their organizations (Kuykendall, 2010; Lieu, 2005;

Schmidt, 2009).

Without a designated agency collecting and analyzing these votes, the total number of presidents who received these votes is also unknown. For example, the American Association of Community Colleges (AACC, 2006) surveyed over 545 community college presidents, and when asked about this leadership crisis, these leaders provided very little insights about these votes. Meaning, from these 545 presidents, representing $61 \%$ of all community college presidents at the time, only $3 \%$ of these leaders reported having received a vote after poor evaluations 
concerning their performance (Vaughan \& Weisman, 2007). This data was also dependent on the leader's willingness to self-report this information. Only a few of these leaders disclosed that they received a vote and described it as a crisis event during their leadership that was precipitated by other events going on in the institution (Vaughan \&Weisman, 2007). It is important to note two possibilities: first, is that it is possible that more leaders who did receive a vote did not report during the survey, and second, that this self-reported data identified the leaders affected in this sample population. For the most part, without a designated agency charged with investigating and collecting data directly from these institutions, the knowledge gaps surrounding possible trends, meanings, and outcomes of these votes will remain elusive. Thus, understanding the extent to which higher education leaders are experiencing this crisis of a no confidence vote on their leadership is in itself problematic and knowledge gap this study hopes to fill.

\section{Utilizing a No Confidence Vote}

An ostensible decision to raise a no confidence vote motion against the leadership is often considered to be an acutely complex process complete with many other challenges facing these organizations (Tierney, 2007; Wright, 2007). For instance, a primary characteristic of some of these votes is that it is considered a "last resort in a bad situation" only after sponsors claimed to have previously raised several concerns with or about the leadership prior to pushing this resolution 
forward (Schmidt, 2009, para. 10). Meaning, these votes appear to grow out of the grievances of the institutional players who have placed the blame squarely on the leader for allegedly not being responsive to their concerns. For instance, as these institutional players concerns grow on account of institutional problems such as extensive furloughs; faculty layoffs; lack of pay increases; strategic plans that would infringe on their academic freedom in the classroom, and if the leader appears to be unresponsive to these concerns, then such leadership behaviors can ignite the fuse for a no confidence vote (Bahls, 2014, Kuykendall, 2010, Schmidt, 2009).

With these grievances in mind, institutional players may perceive the leadership as being ineffective in reducing their concerns, and may grow highly frustrated, criticize and evaluate these leaders as ineffective because they failed to alleviate such concerns (MacTaggart, 2012). As a result, these votes may include a potent demand for the leader to exit their institutions (Schmidt, 2009; Wright, 2007), further demonstrating the sponsoring group's ultimate dissatisfaction with the leadership. However, it is unclear if such perceptions about the leadership were substantiated with evidence about the leader's poor performance before the votes were enacted, although most boards may respond with an evaluation of these allegations against the leadership after the vote is raised (MacTaggart, 2012; Schmidt, 2009). From these reports, it is also unclear if these boards were able to substantiate the basis for these critical assessments of the leader. 
These votes have also been described as a highly strategic method in the academy, particularly when these votes are able to garner the attention of the administration and the public about the leadership and tensions in the organization (Schmidt 2009; Wright, 2007). While others suggest that the sponsoring groups need to be cognizant of the seriousness of such critiques against the leadership and the potential costs it can incur on themselves, particularly if boards and these leaders retaliate against them (Tierney, 2007). Essentially, these authors have posited that the outcomes of these votes can be unpredictable for both the institutional players who use them and the leaders who are targeted by them. Overall, several proponents and opponents' narratives were found concerning the decision and appropriateness for using these votes against the leadership; however, a common theme among these writers is that this mechanism is highly effective in drawing attention to these institutions and their leaders, particularly when these votes are publicized and reported in the media (For more details, see Schmidt, 2009).

A highly publicized example of a no confidence vote with many idiosyncrasies surrounding the situations that led to a vote can be seen in the case of Lawrence Summers at Harvard University. Summers allegedly received two separate no confidence votes from over 200 faculty members for each motion (Abel \& Bombardieri, 2005). Summers received his first vote, on the heels of allegedly offensive remarks he made against the trajectories and aptitudes of women in the 
fields of math and science (Hernandez, 2006). The second vote reflected a dissatisfaction with his overall leadership style and actions that resulted in the departures of an influential faculty member, a dean, as well as his involvement in a federal fraud case with a colleague (Hernandez, 2006). Although Summers would eventually apologize for his statements as well as negotiate solutions for myriads of other problems between himself and other constituency groups, a cross-section of the faculty still voted to demonstrate their loss of confidence in his ability to lead the organization (Brush, 2006). This was Harvard's first no confidence vote in its 376 years of existence; therefore, this highly publicized and monumental event would not only influence the opinions about its leaders but tarnish this pristine record within the leadership of the institution (Finder \& Rimer, 2007). Summers would later resign from his position at Harvard, ending his short five-year tenure as Harvard's president (Bombardieri \& Sacchetti, 2006). Still, from these reports and other articles, it is unclear if Summers left his organization solely as a result of the pressures related to these votes or if the votes merely contributed to his decision to exit the institution.

To this point, a major and precarious observation made about the articles reviewed for this study, was the missing voices of other constituents within the organizations, such as staff or students. More importantly, even less was found and is known about the leaders who received these votes during their careers and how they experienced or survived such crises in their leadership. This silence appears to 
be typical of leaders in these situations and in these reports. As a result, these votes remain a phenomenon to be explored by carefully deconstructing the many components that could lead to an endorsement of a no confidence vote against the leadership as well as to understand the potential implications on these leaders and their institutions. Furthermore, it is unclear how these votes could also compound some of the current leadership gap challenges facing higher education, including California's community colleges, which needs further investigations. Therefore, the aim of this study is to better expose the votes' profound impact on leadership in these institutions and explore the potential meanings and implications of the votes on leaders in community colleges and in their overall careers.

\section{Definition of No Confidence Vote and Crises}

A no confidence vote resolution, also known as a motion of no confidence (NCV) or a censure, is also being operationally defined as a semantic tool or mechanism used by the faculty or special interest groups to express their concerns about the administration or leadership within their institutions in higher education (Schmidt, 2009; Tierney, 2007). These sanctions are almost exclusively sponsored by the faculty (and to a lesser extent unions and boards) to demonstrate their loss of confidence in the leadership or administration of the organization, for any number of reasons (Schmidt, 2009; Patrick, 2007; Smith, 2003). Used as a tool against the institution's administration, Patrick (2007) explained how more faculty and unions may be using this practice as a "tool to oppose" the leadership, for any given 
reason, including general disagreements, and frustrations with the leader's direction for the institution (p. 52). These votes are often communicated to the public and the media by the sponsors of these votes, and as a result, they invariably promote the voices of the constituency groups in conflict with leadership (Schmidt, 2009).

\section{Background of the Problem}

Over the past decade or so, most higher education practitioners and educators have grown accustomed to emerging reports in trade and professional journals about no confidence votes (NCVs) being used as a mechanism against the leadership in the academy. Mooney (1998) speculated that during such incidences, the institutional players have simply lost their confidence in the leadership, and such leaders are perceived to be distracted by other pressing demands and responsibilities other than those of the institutional players. As a result, she further posited that such interactions between the leadership and constituents can either result in war or dialogue between these two groups (Mooney, 2008). Even with such increased accounts and speculations of these votes, it is still unclear how many institutions are using these votes, why they use them, what they mean, or when such votes were first utilized against the leadership in higher education in the United States. Historically, the no confidence motion was adopted from the British parliamentary form of government, which held its first vote against Prime Minister Frederick North, who was forced to resign in 1782 (United Kingdom Government 
Archives, 2009). The Parliament, at the time, voted to force North to resign after the British suffered heavy losses in Saratoga and Yorktown to the newly organized Americans as well as to demonstrate their dissatisfaction with the handling of the Gordon (Catholic) Riots by said current political party (Schmidt, 2009; United Kingdom Government Archives, 2009). After the successful vote, the opposing party of that government was prepared with a succession plan for parliamentary leadership.

Today, no confidence votes (NCVs) are used nationally and internationally, in such various contexts such as police and fire departments as well as in education (Berger, 2002; Dobruck \& Fry, 2015). The focus of these votes is on the leader's poor leadership performance, style and communication practices during times increased instability or ambiguity that leaders failed to manage (Schmidt, 2009). These perceptions about leaders in higher education can result in them being targeted, and such leaders can include presidents, provosts, or deans, and the sponsors of the vote could include specific faculty, staff, or faculty groups, like unions, for example (Schmidt, 2009; Smith, 2003). Similar to the historical usage of these censures, these votes not only draw attention to the growing concerns and sometimes to the chagrin of the faculty but they often demand the immediate ousting of the leadership (Smith, 2003). However, little is known about these votes in general; how many institutions have used these votes, how these votes can be 
used to legally mitigate against the leadership, and what their potential outcomes and implications are in these institutions.

\section{No Confidence Votes Data}

Even with increased arguments about the proliferation of these no confidence votes in higher education (Schmidt, 2009; MacTaggart, 2012), these writers and others still left these sanctions under-examined and unanalyzed, except for one unit of analysis about California's community college completed by Cindra Smith (2003). As a result, this study was unable to locate any comprehensive national data or significant number of studies that has investigated these votes or responded to these questions raised about the vote. In light of this severe dearth of research and data on no confidence votes in higher education, this study was able to gather and provide a brief analysis of a small sample of NCVs reported in these journals and newspapers.

Nationwide no confidence vote data. Using a search in these trade journals, this study focused on gathering a sample of no confidence votes where enough information could be ascertained about specific incidents and situations that resulted in these votes, as well as reports that could provide some knowledge of causation and implication for these votes on the leader and organization, which were all invaluable to this review. As a result, a small sample of NCVs $(N=134)$ included colleges and universities nationwide and was used to explore the usage 
and potential trends in NCVs over time as well as the rationales that were given for these votes. These specific cases chosen were based on the earliest reports of the vote in 1989 to present throughout the United States. Consequently, Figure 1 provides an overview of these votes among presidents by gender in higher education nationwide. In addition, by mapping the locations of each institution that gave its leader a vote across the United States, Figure 1 also provided a snapshot of where and when these votes have occurred throughout the nation.

From this small sample the data illustrated that, since the 1990 s, a no confidence vote has occurred at least once throughout 42 states, which may not indicate a trend but it at least connotes widespread usage nationwide. Without the availability of primary source data and analysis, it is unfeasible to substantiate the evidence that a trend is actually occurring in higher education nationwide or within specific sectors or regions of education, as noted by Narissa Polonio (Lane, 2002). Likewise, Figures 2 and 3 further illustrates who received a NCV by gender and the number of votes cases reported over time from this sample. In relation to gender, it is difficult to determine if either gender was more targeted by the vote during any specific time, although proportionally there are more men than women in these leadership positions. Each president's gender was confirmed through internet searches and college catalogs. Additionally, this data sample was limited in providing insights about race or ethnicity of the leaders and whether or not one group was more affected than others. In addition, this sample data also implies that 
more votes were reported between 2005 and 2015, although such reports cannot definitively suggest that more votes were occurring during this time. A key finding in this sample, however, is that there appears to be an increase in reporting of these votes, perhaps this due to the increase of several media outlets readily available to constituency groups since the 1990s. In addition, the given rationales and meanings for these votes are also identified and discussed in the figures below.

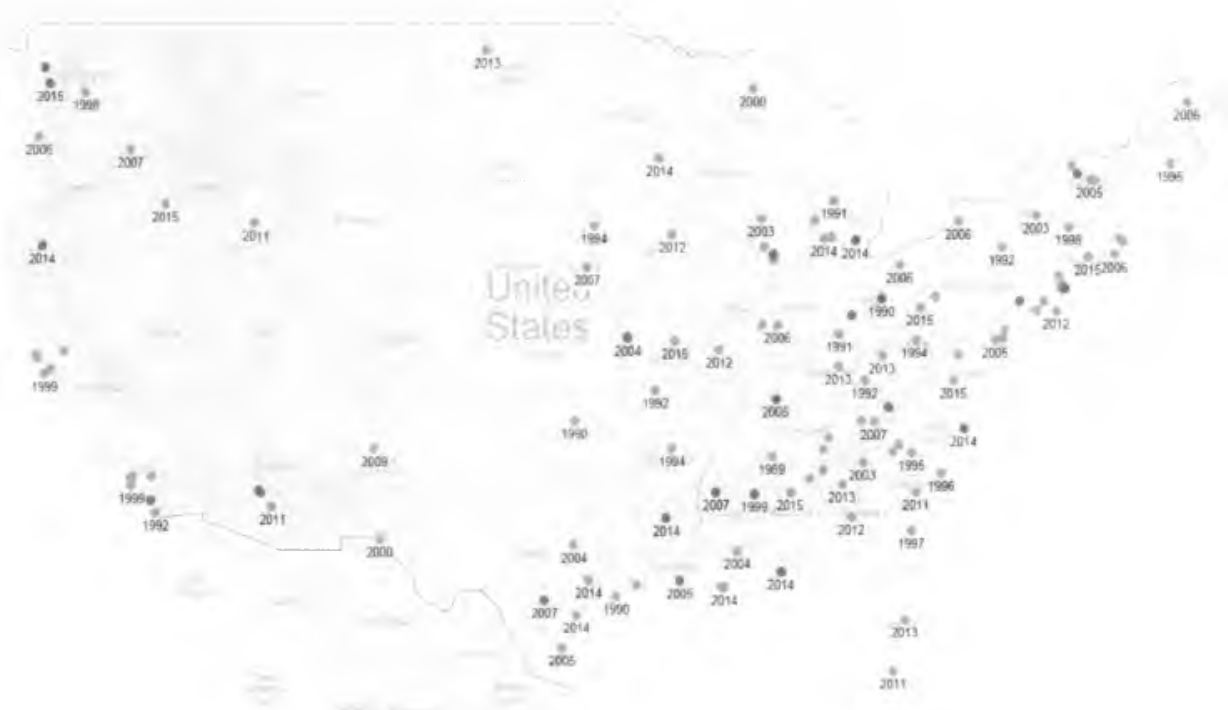

Figure 1. Nationwide Sample of No Confidence Votes Among Presidents in Higher Education by years. Map of the United States consisting of a no confidence vote sample $(N=134)$ from public and private colleges and universities between 1989 to present. Each NCV case is color coded by gender, with the color orange representing males and blue for females. Data was generated from cases found on The Chronicle, Inside Higher Education and the Sean McKinniss database and websites. 


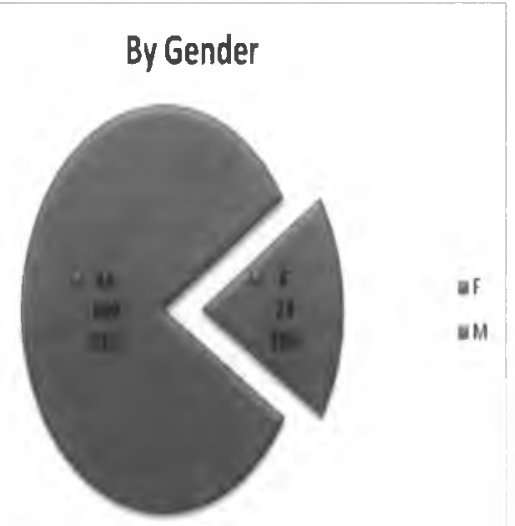

Figure 2. No confidence votes by gender. This figure illustrates the proportional differences in gender from a sample $(N=134)$ of NCVs. From the sample, one or more cases included votes that were not given to a single administrator but to a collective group or body, such as a board or the entire cabinet.

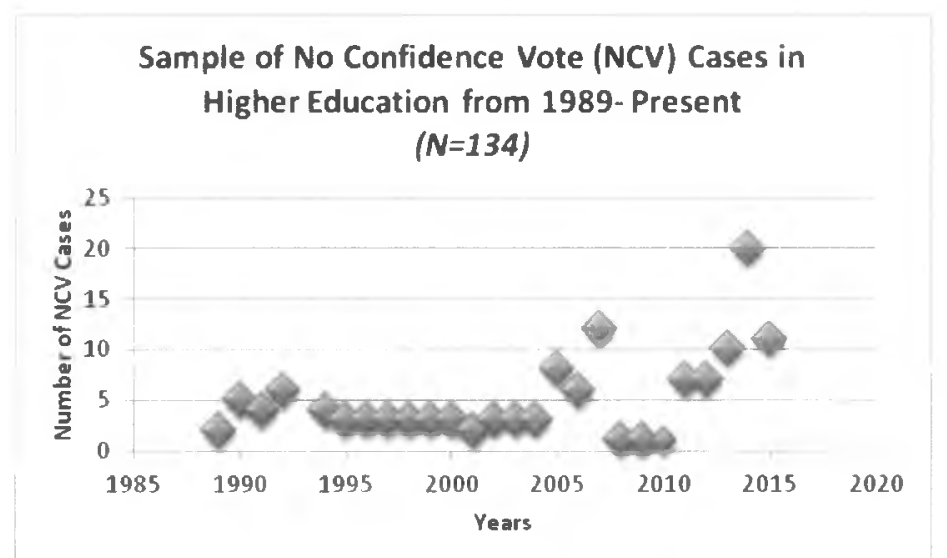

Figure 3. Number of No Confidence Votes since 1989 to Present. This figure illustrates the bivariate data of the number of NCV cases reported over the last 35 years.

\section{Rationales for a No Confidence Vote.}

A leader's perceived poor performance, whether or not such performance is substantiated with evidence, is the quintessential rationale for a no confidence. Amid the many ambiguities about the usage and frequency of these votes, are 
concerns about the rationales, meanings and outcomes of these votes. Some authors have asserted that the primary rationales steering these votes against these leaders generally stemmed from their perceived lack of communication, violations of shared governance and ineffective leadership styles, which are the three major constructs to be explored in more details in the literature review chapter (Kuykendall, 2010; Schmidt, 2009). For example, from the previous sample of NCV cases reviewed in these reports, Table 1 below highlights these rationales, which are categorically clustered into three major divisions, internal, external and non-specified rationales. It is important to note that these clusters could have been clustered in several different ways and are not limited to these categories.

The term internal rationales is being used here to loosely represent situations that emerged from within the institution and contributed to the concerns raised by the sponsors of the vote, such as the leader's decision making strategy or budget allocation processes. For instance, of all the votes in the sample ( $\mathrm{N}=134), 99(74 \%)$ of these votes were related to problems directly related to the leader's practice of leadership, their style of leadership, and/or the ways they communicate with constituency group during key work cycles. The term external rationales, pertains to the situations that emerged outside of the institution that directly or indirectly impacted the organization, such as the loss of accreditation status or dwindling endowment funding, and for which sponsors of the vote ascribe responsibility to the leadership. Meaning, less than $7 \%$ of the votes in the sample, reported that the 
reputations of the leader and/ or the institution were key rationales for the NCV resolution. Finally, the term no-specified rationales, includes the reports that did not clearly denote a specific rationale for the vote but either announced it or stated that the matters were being addressed privately. These types of reports were particularly interesting because while a report was made publicly and could have serious implications for the leader and the institution, the particulars of the vote were said to be private. It is unclear why these types of NCV pronouncements were even made available to the public or why media sources did not provide follow-up about these incidences. It should also be noted that the number of rationales counted are based on the most significant problem highlighted in each case. However, each case may have had multiple reasons for these votes and therefore containing overlapping rationales that could be applied to more than one case.

Table 1

Rationales for No Confidence Votes Cases from Sample $(N=134)$ 


\begin{tabular}{|c|c|c|c|}
\hline Type & Categorles & Rationales & $\begin{array}{l}\text { Number } \\
\text { of Cases }\end{array}$ \\
\hline \multirow{3}{*}{ Internal } & 1 & $\begin{array}{l}\text { Overall Leadership Style and } \\
\text { Performance and Behaviors, i.e.: poor } \\
\text { communication, micromanagement, } \\
\text { ethics, lack of qualifications or } \\
\text { competencies, personal scandals, etc }\end{array}$ & 45 \\
\hline & 2 & $\begin{array}{l}\text { Shared Governance Violations, i.e.: } \\
\text { failure to consult with faculty in decision } \\
\text { making. failure to be transparent in shared } \\
\text { governance processes, etc. }\end{array}$ & 23 \\
\hline & 3 & $\begin{array}{l}\text { Leadership Decision- Making,i.e.: } \\
\text { financial mismanagement, problems with } \\
\text { wages, hiring and lay off challenges, } \\
\text { flawed strategic plans }\end{array}$ & 54 \\
\hline External & 4 & $\begin{array}{l}\text { Reputation or loss of status, i.e.: loss of } \\
\text { accreditation, loss of significant } \\
\text { endowment, etc. }\end{array}$ & 2 \\
\hline $\begin{array}{l}\text { No } \\
\text { Specified }\end{array}$ & 5 & $\begin{array}{l}\text { No reasons were reported or the instituion } \\
\text { reported that the matters were being dealt } \\
\text { with privately }\end{array}$ & 10 \\
\hline Total & & & 134 \\
\hline
\end{tabular}

Note. Table 1 illustrates the rationales offered from sponsors of the vote and in reports from a sample of no confidence cases $(\mathrm{N}=134)$.

As Table 1 denotes, each of these types of rationales for the votes were often focused on the leader and his or her failure to effectively lead, communicate, or support governance structures within these institutions. However, other authors have suggested that leaders often deny such allegations against their leadership (Schmidt, 2009), and that these leaders' rationales suggested that they were being attacked for doing their jobs effectively and that these votes were symbolic of resistance they faced for their efforts to cajole the faculty to work with them (March \& Weiner, 2003; Wright, 2012). As a result, these leaders maintain that they were indeed effective, tried to mobilize and make changes in their organizations in response to some of the institutional problems, but that they encountered strong 
resistance and opposition from institutional players (MacTaggart, 2012; Smith, 2003).

Thus, such disconnects about the meanings and significance of these votes have resulted into two major narrative streams found in this sample. These narratives are captured in Table 2 below. These are the two main arguments found in the literature, which could also be regarded as comparable to the mindsets that are mostly aligned to the increased political polarization between faculty and management in the academy (Gabriner, 1995). Table 2 provides an overview of some of this specific binary rhetoric between these groups concerning NCVs.

Table 2

Polarized Arguments about the Meanings of No Confidence Votes in Higher Education 


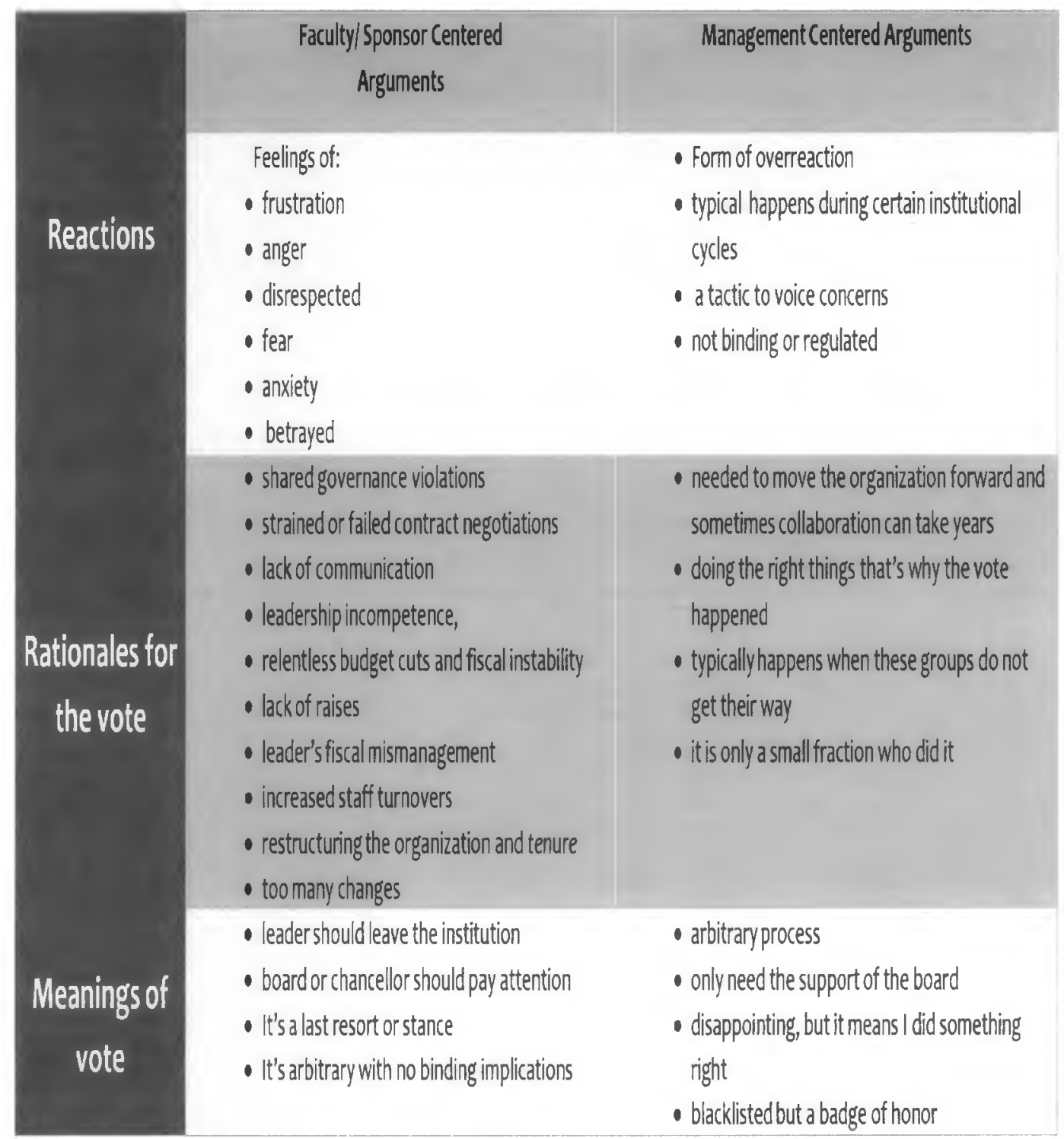

Note. These arguments were taken from a sample of NCV votes $(N=134)$ wherein both the sponsors of the vote and the administration (i.e. the board or spokesperson for the leadership) discussed their rationales about the motion. Data were generated from cases found on The Chronicle, Inside Higher Education, the Sean McKinniss database and Smith's (2003) No Confidence Vote in Community College Study.

Rationales of leaders. Table 2 further illustrates that the responses and reactions of management about these votes and they appear to be centered on the 
politics and symbolic meanings of the vote, as being arbitrary or ineffective in firing them (Schmidt, 2009). Meaning, leaders who face these phenomena are more likely to indicate that such votes occurred when they were acting as a strong leader or change agent within their institutions, particularly when they have the trustee's full support (Brush, 2006; Wright, 2007). In addition, leaders may also be espoused to thinking about these votes as a type of attack on their leadership because they are doing something right and standing up for "what was unpopular with certain groups" (Smith, 2003, p.10). Because of these viewpoints, such leaders do not appear to be identifying these votes as a crisis event on their leadership in these institutions.

The major grievance of the votes is on leadership, specifically on the leader's practice, communication style, overall competencies that were lacking and a leader who is "flawed" (MacTaggart, 2012, para. 16). These votes may be regarded as tactics against the leadership (Tierney, 2007), as well as a type of political posturing against the leadership (March \& Weiner, 2003). Meaning, for many of these leaders, such critical events like no confidence votes, can cause "leadership blues" which are the stories leaders share about the painful experiences during this time and how they survived and persisted in their leadership while maintaining an image of "confidence and strength" (March \& Weiner, 2003, p. 6 and 11). One observation from these arguments is that the voices of these leaders who experienced these votes are usually captured immediately following the 
actually vote and very few articles report on the final outcome or aftermath of the votes or if leaders stayed in spite of the vote or eventually left their institutions, despite their immediate resolve.

Rationales of sponsoring groups. As mentioned earlier, the catalyst for these votes can emerge out of mixture of institutional factors and problems. For the sponsoring groups, whose mindsets may be diametrically opposed to those of the management, their rationales appear to tell a different story from these leaders. These sponsors may view their leaders as a type of public trust, which is a general term applied to individuals who are appointed or elected to a position in government or business with the confidence that they will maintain the trust and respect of those they oversee (Thomas, 1998). As a result, such a trust can be lost or restored by the inactions or actions of the leadership and for any reason. In regards to these votes, it is plausible that the sponsoring groups' rationales may be based on perceptions that leaders are reneging on their commitments to certain philosophies, such as shared governance; neglected their fiduciary responsibilities, and failed to display certain ethics, core values and beliefs that are central to the culture within the institution (Bahls, 2014; Thomas, 1998). Such beliefs and dissatisfaction with leadership may cause the sponsors of the vote to feel validated in using these votes in response to these leaders' alleged malfeasance. These groups may feel justified in using this option, particularly when leaders are believed to violate shared governance policies or procedures, use arbitrary budget allocations 
and appropriations, implement misguided strategies that could change the status quo, or for fail to communicate regularly with constituents and are deemed untrustworthy (Schmidt, 2009; Smith, 2003), for example. Thus, the sponsors of the vote may feel vindicated in using this option against the leadership to demonstrate their growing cynicism.

In addition, there are instances when both the sponsors and recipients of these votes often assert that the vote was arbitrary and it wasn't personal or wasn't a personal attack (Smith, 2003). Meaning, these votes are also viewed as being purely arbitrary and symbolic of the frustrations of the faculty (Tierney, 2007) and that these constituents are justified in using these sanctions to gain the attention of leaders. Similarly, some leaders have offered up similar sentiments and have also suggested that these votes were not personal against them, regardless of the outcome or consequences of these votes (Schmidt, 2009; Smith, 2003). Kuykendall (2010) asserted that even when leaders exited their institutions some time later and after the vote, these leaders often didn't attribute these votes as factor in their decisions to leave.

Overall, both these sponsors and leaders appear steadfast in maintaining their perspectives and rationales for either enacting or surviving these votes, respectively. These mindsets are maintained even though the sponsors of these votes understand that their actions may cause the leader to either stay or go, and leaders may be under pressure if sponsors prevail enough on their boards (Smith, 
2003; Smerek, 2011). Essentially, regardless of the impact or outcome, these researchers further implied that this practice is often viewed as a part of the collegiate culture, and these votes are merely a demonstration of the growing frustrations among the sponsoring groups, wherein these leaders are the ones to bear the blame. However, this study questions such sentiments and assertions about the arbitrariness of these votes, the consequences of these votes on leaders, and widerimplications of these votes on higher education with its current leadership challenges.

\section{Leadership Challenges in Higher Education}

Repeatedly, the literature is very clear about how complex higher-education institutions have become (DiMaggio \& Powell 1983; Farrington, 2008; Meyers \& Rowan, 2006). This is especially true for leaders who must successfully guide their organizations through pervasive challenges, such as budget cutbacks, greater government oversight for accountability, changing demographics, along with a myriad of other crises facing the organization (Malm, 2008; Spillane, Halverson \& Diamond, 2004; Theoharis, 2007; Zusman, 2005). These highlighted issues are believed to contribute to the current problems and challenges facing leadership in higher education in general (Shulock, 2002), as well as the challenges associated with the leadership gaps in the college presidency pipeline, which has been a controversial and pervasive issue in higher education. It is also unclear how no 
confidence votes could further exacerbate the problems concerning this leadership pipeline and leadership in general.

One leadership watchdog in higher education, the American Council on Education (ACE), is well known for its leadership reports and for regularly monitoring these leadership challenges. This challenge is two-fold in that it includes both the imminent hemorrhaging of current presidents due to their inevitable retirement (ACE), 2007; Cook \& Kim, 2012), as well as problems in attracting potential and retaining current leaders in the academy (Community College League of California, 2012). For example, recently, ACE (2007) found that of the 3,000 plus presidents who responded to their survey in 2006, "49\% of American college and university presidents were between the ages 61 and 70 years old," and had held the presidential position for an average of 8.5 years (p. 57). In their most recent survey with 1600 president respondents, ACE noted that the percentage of aging presidents 61 years and older in all sectors of higher education increased from $49 \%$ to $58 \%$ in the 2011 ACE report ( Cook \& Kim, 2012). Thus, from these reports it can be inferred that more than half of these presidents, and possibly their corresponding executive teams or cabinet members, who may have been potential successors, may indeed be aging out of these positions. The ACE reports (2007, 2012) not only warned institutions about this pending leadership gap as a result of retirements, but suggested that they implement programs to attract, retain, and 
prepare individuals within and outside of the traditional presidential pipelines to take over the leadership of their organizations.

Research on these leadership gaps also explored the complexities and problems associated with the overall role and tenure of leaders, such as leaders leaving under duress or under fire (Mize, 2011) or leadership turnovers (Coyne \& Coyne, 2007) that can disrupt and cause instability in an organization. For instance, other authors addressed the problems associated with the changing roles and challenging duties of leaders today compared to a decade ago. A few of these changes included finding new funding sources and building enrollments through global markets, as well as being responsive to the many missions of their institutions and system (Cook \& Kim, 2012; Shulock, 2002). Overall, there are many wider issues and challenges currently facing higher education and it leaders, such as enrollment deficits, fiscal crises or other economic issues that leaders and their boards must attend to, too many to list here (Shulock, 2002). However, it is unclear how a vote of no confidence (NCV) can further compound such prevalent crises or affect leaders and leadership in higher education (March and Weiner, 2003; Schmidt, 2009).

\section{Context of the Problem}

California's community college (CCC) system is the chosen system for this research study's investigation and analysis because it was one of the few systems with limited data available for analysis to build on that is also facing similar 
leadership challenges and concerns about these no confidence votes in higher education overall (McNair, 2010; Smith, 2003). Known as one of the largest systems in the nation, the California Community College (CCC) system boasts 113 colleges in 72 districts with an enrollment of over 2.9 million students and has solidified its place within the higher education environment, as dictated by the California Master Plan for education (Chancellor's Office, 2012). However, this complex system, with its many levels of leadership and diverse student population with different needs, is no exception to having challenges at both the organizational and the leadership levels. For example, this system has remained the least funded in its sector in the state, especially after sustaining several years of budget cuts and high enrollments (Shulock, 2002). Several news and trade journals that cover the CCC system, such as Inside Higher Education and the American Council on Education ( $A C E$ ), have suggested that among the many challenges facing this sector and others, the most salient also involves gaps in leadership, similar to the gaps previously mentioned. CCLC (2012) characterized these leadership positions in CCC system as being so "complex" and "challenging" that boards are opting to recycle leaders who have previously served as presidents or chancellors and who already have the "knowledge" and insights about serving in these types of systems (p. 5). This pattern of recycling leadership may also have further implications on the hemorrhaging leadership pipeline, were presidential candidates, including leaders who have been underrepresented in these positions such as women and individuals 
of color, are no longer interested or pursuing such positions (Gonzalez, 2010; Switzer, 2006).

\section{Leadership Challenges in California Community Colleges}

Similar to the two-fold leadership challenges in higher education, the CCC system is also believed to be in dire need of attracting and retaining individuals for the presidential positions in their system to fill this gap (American Associate of Community Colleges (AACC), 2005; CCLC, 2012). According to McNair (2010), this leadership gap problem was so grave that the State removed restrictions on California State University (CSU) systems allowing them to offer terminal degree programs (EdD) in educational leadership. This move was seen as an effort to create new pathways that would attract potential leaders and improve the pipeline to the presidency (under SB 724), (McNair, 2010).

The promise of this new pathway to the president has struggled to demonstrate its potential returns in closing this leadership gap. For instance, in a recent report from the Community College League of California (CCLC, 2012), it was stated that the leadership gap in this system is believed to still be pervasive and that the recycling of leaders is also a current practice. Therefore, although this state's response to this issue is via terminal degrees and at first glance is commendable; with only 12 additional approved programs to address this problem to date, no data was found to confirm if this strategy has indeed reduced these 
leadership gaps in this sector or others in the state (California State University, 2011).

In addition to the aforementioned leadership challenges facing this system, the California Community College (CCC) system is also facing a retention problem. The tenures of the presidents and chancellors (CEOs) have declined from an average of 5.5 years in the mid-1990s, to 5.1 years in 2011 , and finally to 4.6 in 2012 (CCLC, 1995; 2012). Apart from leaders aging out of the system, the CCLC (2012) also attributed these tenure rates to turnovers, advising that presidents and chancellors (CEOs) are also leaving at higher rates and under duress at $32 \%$. These situations include being fired or having left their positions "under fire... [meaning] because they their contracts were not renewed" or were cut short for other reasons, including budget constraints and the current national recession (CCLC, 2012, p. 3). In a more recent report in $2010,20 \%$ of community college presidents $(n=646)$ surveyed both in California and in other states reported having ended their presidential tenure under "duress," including pressures from groups within the institution (Mize, 2010, p.3). While these leaders were not specifically asked about no confidence votes, it can be inferred that such motions may have occurred $\mathrm{R}$. Mize, personal communication, July, 26, 2011). This particular agency and their reports were invaluable to this study and will be referenced throughout this research to shed light on this phenomenon. 


\section{California Community Colleges No Confidence Votes}

Although the prevalence and incidences of no confidence votes are unavailable in the mainstream data, this research did find one empirical postdoctorate study concerning no confidence votes in California community colleges as well as reported incidences in the news and other journals. For example, from Google searches on California's community colleges (CCC), this study was able to locate in 39 institutions with over 54 separate no confidence vote incidences, publicized in news reports and news cycles in this system, from 1990 to present. Figures 4, 5 and Chart 1 further highlights how many institutions among the 113 colleges have used these votes, the gender of the leaders who received these votes and the number of cases found. For more details on the actual data on these specific cases, see Appendix E, p.505.

\section{No Confidence Votes in California Community College \\ 1990 -Present}

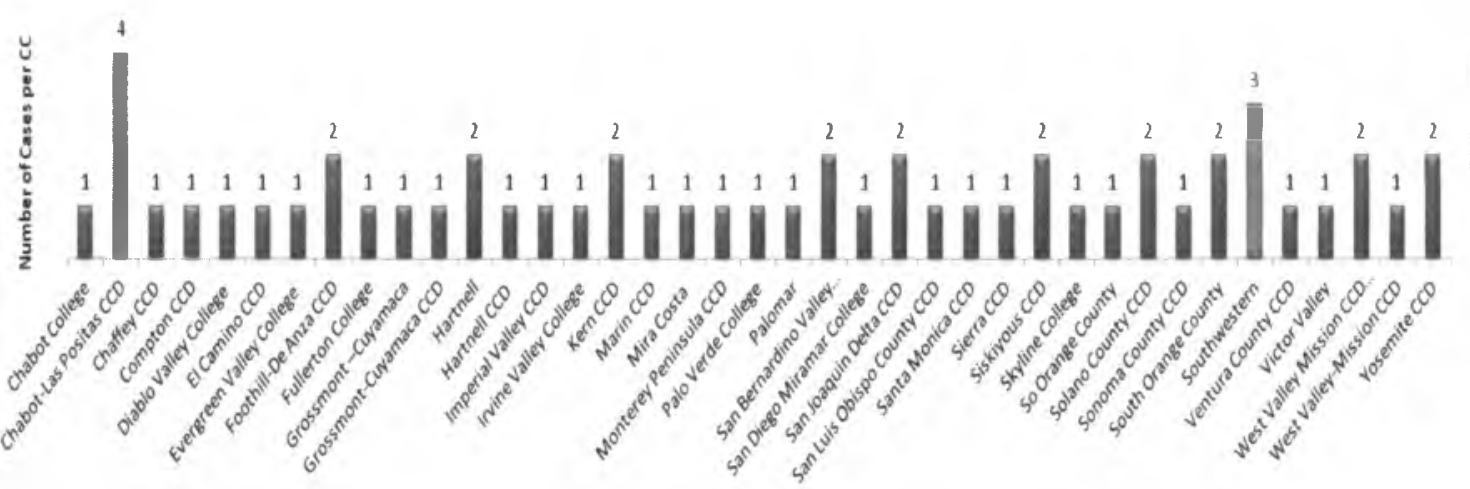

Figure 4. California Community College No Confidence Vote Cases (Total NCVs= 54) Per Institution (Total Institution= 39) Reported in the Media from 1990 to Present. All cases found were from internet searches of each institution within the CCC system. 


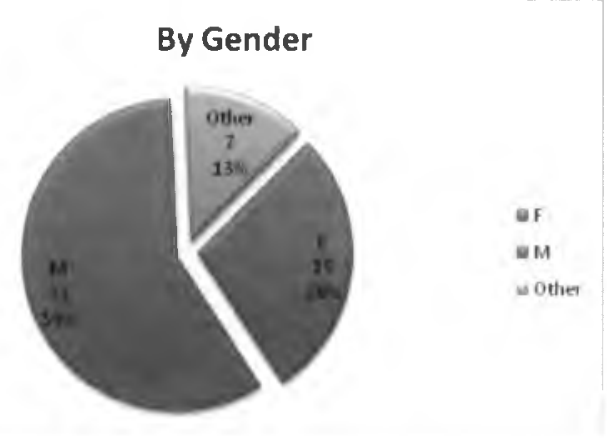

Figure 5. California Community College No Confidence Vote Cases by gender from 1990 to present. All cases found were from internet searches of each institution within the CCC system.

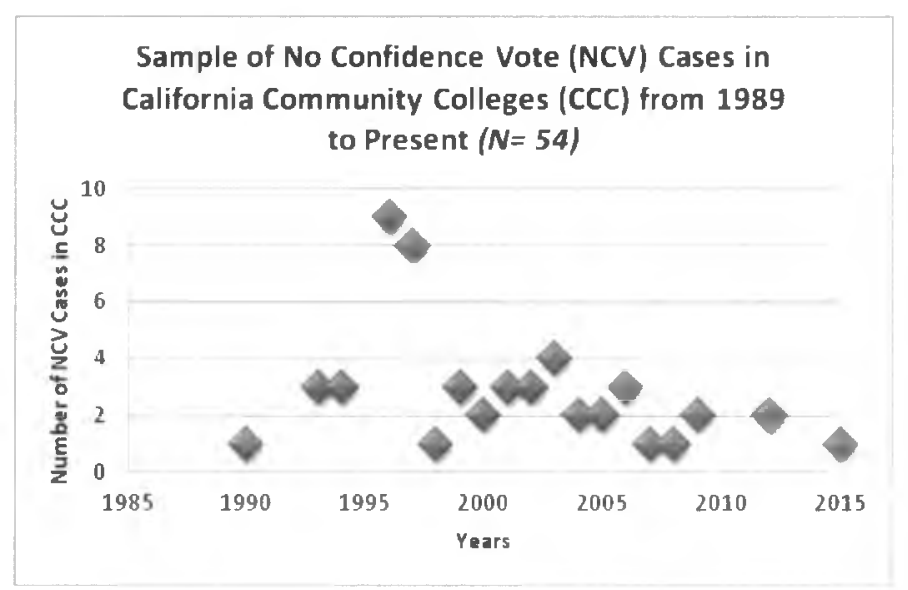

Figure 6. California Community College No Confidence Vote Cases Reported in the Media from 1990 to Present. All cases found were from internet searches of each institution within the CCC system.

This data sample further illustrates that of the 113 community colleges in this system almost $39(35 \%)$ community colleges used a no confidence vote one or more times. As a matter of fact, for Chabot-Los Positas Community College District, one source cited that between 1994-1998 three of their presidents each 
received a no confidence vote, consecutively, from the faculty within that district (Rhoan, 2007). In addition, each leader's gender was confirmed through internet searches of college catalogs and resumes. Concerning gender, this data highlighted the point that both genders were experiencing these votes against their leadership, although the actual number of all male and female leaders during this time and in higher education overall was unavailable. Smith (2003) also speculated that different marginalized groups may have been getting these votes at a faster rate in their tenures compared to their white counterparts. No further evidence was provided to substantiate this claim. It is important to note that $13 \%$ of the votes were directed to collective groups of individuals, such as the leader and cabinet, the leader and board, for example. In addition, there appeared to be a major increase in the number of votes given to leaders between the years of 1995-1996, which one scholar attributed to in-fighting concerning budget appropriations and boiling points reached over shared governance directives being violated by leaders (Smith, 2003). With such a small data set found, it is also unclear if there is indeed a trend or uptake in votes in the CCC system as well.

\section{Impact of No Confidence Votes on California Community Colleges}

Smith's (2003) report, which was completed under the leadership of The Community College League of California (CCLC), also mirrors some of the findings in the national data. The CCLC serves as an advisory agency to California 
community college's administration and boards and is known for tracking these votes as well as the tenure of leaders and leaders who leave their institutions under duress, which could include departures due to NCVs. Smith (2003) not only provided an overview of the number of no confidence votes that have been reported to the CLCC over ten years, but she included probable impact of the votes on the organization and its leaders. This report included an analysis of the 35 presidents and chancellors (CEOs) who received at least one no confidence vote between 1993 and 2003 in the CCC system. This report generated much interest for this researcher, and it will be used to provide some general knowledge of these votes within this system.

\section{Sponsors and their rationales for NCVs in California's community}

colleges. In her evaluation, Smith (2003) posited that of all the votes reported to and analyzed by her agency were enacted by a faculty senate and that these votes were directed to presidents over college(s) or chancellors over districts or systems. Table 3 provides a breakdown of those leaders who were targeted by the vote. In the same vein, Smith (2003) also outlined the genders of the leaders who received these votes; see Figure 8, for the reasons mentioned previously. An interesting finding in Smith's (2003) analysis of no confidence vote cases in California community colleges were the four major context specific and contributing factors for enacting the vote: (a) resistance to organizational and administrative changes; (b) disagreements over collective bargaining negotiations; (c) violations around shared 
governance or decision-making processes between the leader and faculty, and (d) loss of trust and confidence in the leader's overall leadership competencies, style, or practice. Figure 7 provides an outline of the number of the cases that were attributed to these factors. Smith (2003) also asserted that these votes emerged from conflicts resulting from the daily activities and work cycles in these colleges, wherein most faculty and administrators found it increasingly difficult to lay aside their own agendas and self-interest to make the needed decisions and compromises in this process.

Specific challenges within California's community colleges. Smith (2003) also highlighted a major abnormality in the rationales for no confidence votes in 1996 (with eight votes), in 1997 (with seven votes), and again in 2003 (with five votes). The researcher observed an unexpected shift in no confidence vote patterns, particularly after California community colleges received a huge influx of state funding following years of budget constraints (Smith, 2003). Based on her analysis, Smith (2003) speculated that the cause for the increase could have resulted from both new laws and policies about shared governance which became an added element in the brewing strains and political posturing between these groups, which now required them to share in the decision-making about specific issues. In addition, she suggests that there may have been growing tensions over the leader's decisions to allocate or reallocate these resources to specific groups within the organization (Smith, 2003). However, R. Stanback Stroud speculated that perhaps 
these votes are a new form of performance evaluation in lieu of the former Morale Survey (R. Stanback Stroud, personal communication, Dec 16, 2015). Institutional players within the CCC system were used to using Morale Surveys to provide feedback about their satisfaction levels with leaders and the institution, such as how is the institution operating and about the effectiveness of the leadership. These surveys were presumed to have ended in the mid-1990s. Perhaps, with this survey removed, the implication here is that these votes were the new ways of getting the attention of the leadership and a way for the faculty to raise their voices.

\section{Implications and outcomes of NCVs in California's Community College}

system. Concerning the implications of the vote on the leadership in California's community college system, Smith (2003) proposed that these votes occurred whether or not the institutional environments were highly collaborative or resistant towards these leaders. In addition, from her assessment, it appears that these votes may or may not be based on the leader's overall performance, competencies or abilities (Smith, 2003). Although, she did not make any direct references to the implications on the organization, other than boards finding ways to mediate the process between the sponsoring group and the affected leader, she also asserted that more research is needed to understand the effects of these votes on leadership within California's community colleges (Smith, 2003).

Smith (2003) also asserted that the outcomes of these votes are also wideranging. Consequently, she observed that "two-thirds" (23 of 35) of all leaders who 
received the vote "remained in their institutions for at least 2-7 years," while the other third left within a year after the vote (Smith, 2003, p.6). Similar to the national data on these votes, these leaders also appeared to leave for personal reasons or because of another job opportunity, but none appeared to leave as a result of the vote. Similarly, Smith (2003) also pointed out that the rationales and factors leading to these votes were also varied, and more research is needed to deconstruct such rationales and attributable factors. These data points are further outlined in Table 3 and figures below.

Table 3

No Confidence Votes Reported to California Community Colleges

\begin{tabular}{|l|l|l|}
\hline $\begin{array}{l}\text { Votes directed to 35 } \\
\text { leaders: }\end{array}$ & $\begin{array}{l}\text { Number of votes } \\
(\%)\end{array}$ & Primary Rationales for the Votes \\
\hline $\begin{array}{l}\text { CEO/ Chancellors over } \\
\text { Systems }\end{array}$ & $28.6 \%(10)$ & $\begin{array}{l}\text { Leadership } \\
\text { Collective Bargaining } \\
\text { Disagreements }\end{array}$ \\
\hline $\begin{array}{l}\text { Superintendents/ } \\
\text { Presidents over districts }\end{array}$ & $48.6 \%(17)$ & $\begin{array}{l}\text { Leadership } \\
\text { Organizational and } \\
\text { Administrative Changes }\end{array}$ \\
\hline College Presidents & $22.8 \%(8)$ & $\begin{array}{l}\text { Leadership } \\
\text { Participation in Decision } \\
\text { Making or lack thereof }\end{array}$ \\
\hline
\end{tabular}

Source: Smith, C. (2003). Reported Votes of No Confidence in California Community Colleges from 1993-2003. 


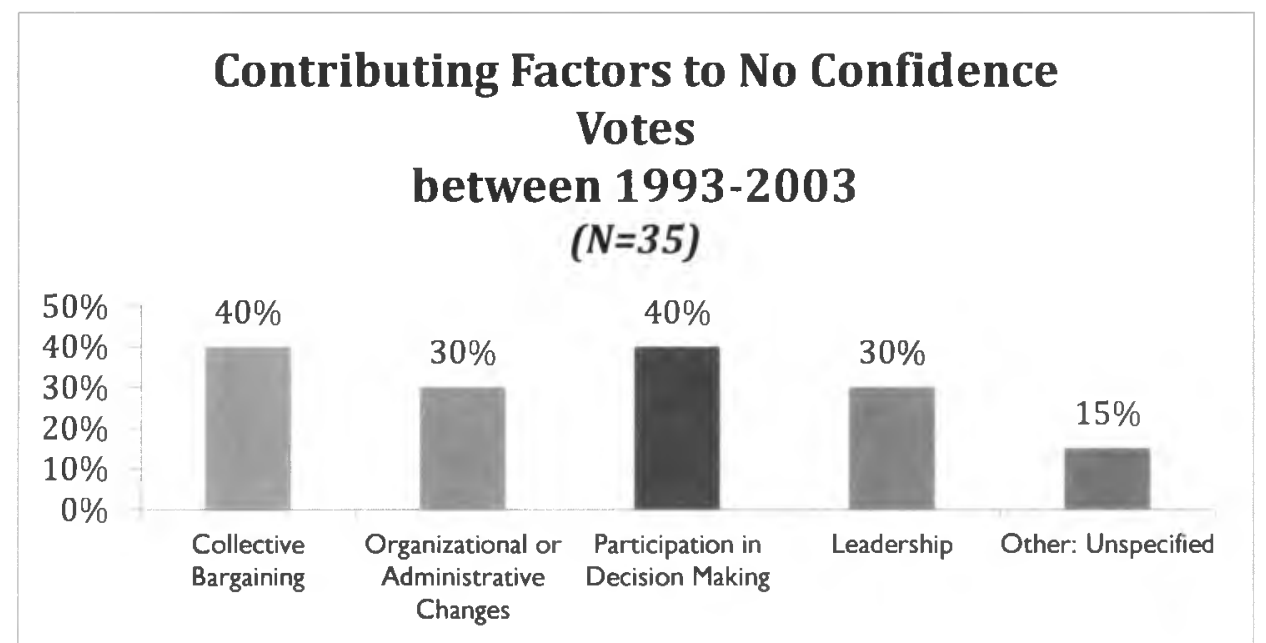

Figure 7. Contributing factors to no confidence votes in California community colleges from 1993-2003. Source: Adopted from the Community College League of California (CCLC) Report by Cindra Smith in 2003.

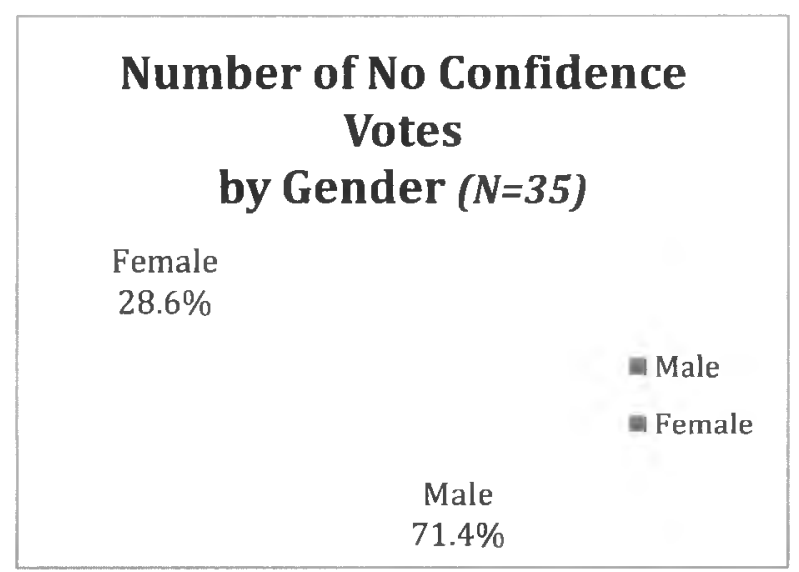

Figure 8. Number of NCV by Gender. Gender divisions between men and women who received a no confidence vote while serving as presidents in California community colleges from 1993-2003. Source: Adopted from the Community College League of California (CLCC) reported by Cindra Smith in 2003.

\section{Direct impact of NCVs on presidents in California's Community}

College system. Another interesting finding in Smith's (2003) report was that it 
was one of the only documents that provided insights about the impact of the vote on leaders themselves and their reactions to it. Smith (2003) presented information about the leaders' initial reactions to the votes on their leadership. In each of the cases she explored, the leader's reaction to the votes varied from "anger, depression, increased illness, difficulty sleeping, defensiveness, and a loss of trust and respect for the faculty leaders" (Smith, 2003, p. 9). While she did not conduct in-depth interviews with these presidents, such leaders may have presented a different interpretation of the factors that led to and caused the votes, as well as what influenced them to stay or leave their organizations beyond their board's support. Essentially, Smith called for further research to investigate the individual circumstances and rationales for these votes, with an emphasis on the narratives of the leadership and context specific factors that contributed to these votes.

In summary, although the data on these votes are limited to non-existent, Smith's (2003) empirical work is considered invaluable to this study. Her work has offered some perspectives on this sanction, beyond what has been previously reported, as well as possible contextual factors that may be attributable to these votes. It is important to note that the data points highlighted about these votes are not generalizable to all institutions or community colleges or cases in higher education, but they did present an exemplar analysis to consider in future research, which is also greatly needed. A major critique of this study, however, is that it did not capture other possible antecedents or antecedent conditions, beyond budget cuts 
and governance issues, for example. Such factors are considered the established "conditions leading up to or cueing behaviors," and the "conditions from which conflicts are likely to emerge" respectively (Schermerhorn, Osborn, Hunt \& UhlBien, 2012, pp.14, 58). Shen and Cho (2005) suggested that such antecedents could exist in the organization before the leader took over the organization, such as ingroup fighting or other conflicts or concerns. In addition, Smith's (2003) study also failed to capture the full scope of the aftermath of these votes on the institution and the presidents who received the vote, as well as how they made sense of these votes on their leadership, a gap this study is positioning itself to fill.

\section{No Confidence Votes as a Phenomenon}

Beyond the limited scholarship and lack of data about no confidence votes and its impact on these current leadership challenges, these no confidence votes are being considered a phenomenon because of the virtually non-existent voices of the leaders, who experienced this crisis on their leadership. Meaning, these reports failed to delve into the ways these leaders experience such crisis on their leadership and how they make sense of it.

The researched news reports concerning these votes also highlight these leaders' silence concerning the vote. For instance, the San Diego Union-Tribune (U-T) reported that an embattled MiraCosta Community College President was being ousted from her position as a result of "Palmgate" a scandal involving the 
illegal sale of a single palm tree (Lavelle, 2009; Saaverdra \& Sherman, 2007). This report came almost two years after a campus upheaval over the scandal alongside a lengthy investigation into the incident, which cost the institution close to $\$ 2$ million dollars (Lavelle, 2009; Saaverdra \& Sherman, 2007). The costly investigation was launched by President Dr. Victoria Munoz Richart, who later received the no confidence vote (106 to 8 ) from the faculty against her leadership and handling of the incident (Lavelle, 2009; Saaverdra \& Sherman, 2007). According to the $U-T$ press, this scandal, coupled with the pressures associated with the vote, may have resulted in the president's resignation after a short-- three year-- tenure (Lavelle, 2009). Ironically, throughout the nearly two-year incident, the voice or narrative of the president concerning the scandal and the vote of no confidence remained virtually silent in the media; meanwhile, those of the faculty, staff, and board were readily available in these reports.

Although this research acknowledges that most organizations appear to survive these votes regardless of whether the leader stays or go, it is difficult to substantiate this claim, particularly when the information reported to the media usually includes only speculations about the aftermath and consequences of vote. March and Weiner (2003), after assessing situations that can result in a crisis in leadership in higher education, contended that these types of crises (including no confidence votes) are part of the leadership reality in the academy, wherein "leaders reap the rewards of public satisfaction [and at the same time] they bear the pain of 
public unhappiness" (p.5). Therefore, this study asserts that the public implications of such censures are fundamental for understanding leadership in its context (the academy) and the overall impact of such crises in leadership that can occur as a result of these no confidence votes. Even with such reports, it is still unclear what the consequences are on leaders; perhaps, this is because few leaders will experience this particular crisis during their time of leadership.

In summary and from the articles reviewed, this silence in these reports appears to be recurrent among these leaders in these situations. From some of the reports investigated, the mention of the leader's feelings is limited to statements from these leaders' boards or the designated spokesperson who would advise their institutions and the media that the targeted leader had no intentions of leaving the institution or that such leaders are working with the sponsoring groups concerning the matters raised privately (for examples, see Wright, 2007). However, for the most part the voices of the leaders were absent in these follow-up reports. Perhaps, this omission is because these leaders either declined to make statements or due to some potential legal implications associated with these votes. As a result of their missing voices in these reports, little is known about the targeted leaders who experience a no confidence vote, the impact it can have on their leadership and the leadership of their organizations, a gap to be reduced by this study. Overall, little is known about the potential and perhaps, far-reaching ramifications of NCVs that can further and indirectly limit the tenures of leaders, set precedence about the political 
climate in that institution, as well as contribute to this leadership challenges in California's community colleges.

\section{Description of the Problem}

Thus far, this chapter has highlighted some of the other salient problems associated with no confidence votes, which include:

1. The lack of adequate data to analyze these votes.

2. A lack of knowledge about how and when these votes are to be used in organizations.

3. The impact of these votes on the leaders and their organizations (during and after the votes).

4. The implications of these votes on the organization and other constituency groups.

5. The effects of these types of votes on the pipeline to the presidency, especially on potential and current leaders.

Furthermore, little is known about the catalysts, frequency, or outcomes of such votes, which appear to be (a) increasing, perhaps as a result of increased reporting of the votes in professional journals; (b) under-investigated, evidenced by the limited empirical research data and analysis about these votes, and (c) used as a mechanism for constituents to raise concerns about issues within the organization and the leadership. What is clear from the current literature is that the outcome of 
the vote also depends heavily on the institutional perceptions about the leader's effectiveness, the context-specific factors within these organizations, as well as the reactions of these boards and whether or not they feel pressured to submit to the demands of the faculty or faculty unions (March and Weiner, 2003) or will unequivocally support the president in spite of these motions (Schmidt, 2009).

Overall, little is known about the emergence and ramifications of no confidence votes, and even less is known about the targeted leaders and how they make sense of these votes on their leadership. In summary, these votes appear to act as either the cause of or the effect of a crisis within the academy, particularly when these votes target the leadership. Meaning, the literature pointed out that the top three attributable rationales for the vote were based on the leader's competency, capacity, and ability to lead effective; the leader's practice of communicating and collaborating effectively within the context of the organization, and the leader's ability and responsiveness to managing shared governance relationships. These specific rationales will be examined in more details in the literature review. Additionally, little is known about the aftermath of such votes, particularly if leaders decide to leave abruptly, and even less is known about how these institutions work to stabilize the institution or to sustain or revamp a leadership agenda after these leaders leave (Fain, 2007; Fanelli, 1997; March \& Weiner, 2003). There are many facets to this phenomenon that warrant closer investigation about the impact of the vote on leadership, the situations and context specific 
factors that may be attributable to these votes, as well as the issues and challenges that coexisted alongside these events.

\section{Purpose of the Study}

In an effort to address these research problems, the primary purpose of this phenomenological study is to explore the potentially rich counter-narratives of presidents who experienced a vote of no confidence within the California community college environment and how they made sense of these votes on their leadership. Additionally, this study will examine the context-specific factors that can contribute to these votes in the California community college system, including the impact of these votes on the leader and the organization.

A secondary purpose of this study is to contribute to the knowledge sources concerning these votes and how they can be further precipitated by other antecedent factors in the institution or accelerated by challenges in the leadership pipelines in these community college environments. It is important to note here that whether or not one agrees that these votes are a justifiable option for raising concerns about the leader is not within the scope of this study or a purpose of this study. Consequently, this research proposes to add critical knowledge about this phenomenon to the leadership and crisis management literature about the presidency. 


\section{Research Questions}

The development of the research questions stemmed from the search of the preliminary research data on crises in leadership, including the few empirical and doctoral studies that tackled these votes in case studies and other qualitative work. In most of these studies, the sensemaking approach designed by Karl Wick (1995) is an approach by which researchers examine how individuals in any environment make sense of themselves, attempt to interpret their roles in their organizations, and/or try to understand the role of their organizations in their lives. It is an approach that garners the information needed from key informants about their constructed understandings of situations that happen to them (Wick, 1995). The sensemaking approach in research is well-documented as an appropriate mechanism for helping participants reflect on and construct their experiences about a situation only they experienced (see Eddy, 2005), and it is has been well cited and referenced in many studies. Sensemaking is also aligned with the purpose of the qualitative research design, which includes "gaining an in-depth understanding" from participants and to understand "their perspective" (Patton, 2001, p.29).

Using Weick's (1995) sensemaking theory will allow participants to "make sense of" their realities about a phenomenon they experienced, which researchers can then use to compare with other available sources (p.4). This research approach not only provides a methodological approach for garnering data, but it also offers a conceptual lens by which to analyze and make sense of the participant's perspective 
(Eddy, 2005). Therefore, this study will use this approach as a methodology for gathering the stories about the votes from participants and for analyzing the key components of these stories. In addition to this approach, the following questions are the primary research questions guiding this exploratory study, which will be used to guide the interview questions (see Appendix C, p. 96).

The research questions guiding the study are as follows:

1. How do presidents make sense of a no confidence vote on their leadership?

2. What factors do they attribute to the vote?

3. What lessons did these leaders learn as a result of this experience, if any?

\section{Statement of Ethical Considerations}

This study will seek out potential participants from among presidents who are in sensitive and high-level positions in higher education. As a researcher, I will adhere to the protocol of causing no harm to these and any participants. Although there is usually some loss of privacy for participants who consent to participate in these types of studies, this study will make all necessary precautionary measures to reduce this risk by discussing the details and potential risks of this study with each participant and requiring them to complete and sign consent forms that will clearly denote the details of the study, the possible loss of privacy, and expectations. In 
addition, all identities and identifiable labels will be replaced by pseudonyms to maintain confidentiality.

Furthermore, using the protocols outlined in the literature and by the Institution's Review Board (IRB), the researcher will protect the rights and privacy of all informants by adhering to standards for working with this population and maintaining their data in a secure location to minimize any risk to the participant. This study also allowed sufficient time for member checking with each participant during the actual interviews, where participants were asked specific questions about their individual interview responses for clarification, and to increase reliability in results, they were also asked to comment on information found in other documents, newspapers and journals about their individual incidences. The full ethical considerations for the study can be found in the methodological section of this project.

\section{Justification and Significance of the Study}

This research study is highly significant because it will address the problems and controversies about the meaning of the no confidence vote from the leader's perspective. Thus, this study is also being positioned to answer the research questions posed and will contribute original knowledge to the field and about leadership in community colleges from primary sources. Furthermore, it will work to fill the knowledge gap in the field in an effort to address the wider leadership problems facing community colleges. Although the dominant or master-narrative in 
the leadership literature is focused on crises that leaders must attend to within their organizations but not necessarily crises that are focused on them personally, this research is proposing to contribute new insights about leadership and crisis management to the field. More specifically, knowledge about presidents and their practices in situ is significant for filling the gap in the scholarly literature about these critical issues in the community college context and the leaders who experience these votes (Spillane et al., 2004).

\section{Limitations}

A major limitation of this study is that it will not provide any evaluations concerning the validity of these no confidence votes against the leadership, a key epochè of this study (Creswell, 2007). As noted previously, the goal of this study is to gain insights from those who experienced these votes as opposed to evaluating or judging these votes. As a result, all such judgments and conclusions will be left to those who experience these votes and the readers of this study.

Another limitation is that the knowledge gained from this study is not generalizable to all no confidence vote cases, but some knowledge may be transferable. As a phenomenological study, this research project cannot offer any generalizations about all presidents in community colleges. However, it will add to the knowledge about the unique experiences of presidents who received a no confidence vote, about the outcomes of the votes, and how presidents in the 
California community colleges experience and make sense of these votes on their leadership and the organization.

In addition, another limitation is that the researcher is an outsider in the California community college environment, and she has not personally experienced this level of leadership in her career. As a mid-level university administrator, outsider from four-year institutions, and a woman in the non-traditional leadership pipeline, this researcher was wary of any type of stereotypical threats, prejudices, limitations or gendered biases that may be brought to the study (Bogdan \& Biklen, 2007). To minimize or diminish such biases or threats, this researcher used reflexive data memos and journals to document my thoughts and impressions during my time in the field, especially during interviews. This reduced the possibility of the researcher manipulating the data to coincide with any presupposed theories about what is going on in these situations. In addition to taking these precautions, this researcher will also incorporate a data-auditing and memberchecking process to help limit these threats to the study.

Another limitation of this study is the limited number of possible presidents who received these votes and their availability to participate in this study. One issue is that, overall, the number of presidents who receive these votes is limited, and, without any formal agency collecting these data, finding and locating these presidents may be challenging. In addition, these leaders may or may not be under court order or some other agreement that would prevent them from discussing the 
circumstances around these events. However, this study used a convenience sample of presidents to ensure that an adequate number of participants was attainable.

\section{Assumptions}

A major and general assumption of this study involved the feasibility of the study and if the study would appeal to leaders, who appear otherwise silent in other reports. Meaning, this assumption is that presidents who receive these votes would be willing to speak to me about this highly sensitive and critical juncture in their leadership openly and without hesitation. Another major assumption is topicspecific, meaning that assumptions were made that no confidence votes will have an impact on leaders who stay or go and that this study will discover something new. Similarly, by using Weick's (1995) sensemaking approach, this study's theoretical assumption is that leaders have taken some time to reflect on what they have done or experienced and are able to share a plausible story about these votes.

A third assumption of this study is a philosophical or ontological one, wherein it is believed that each leader's reality and experiences may be similar, independent or different from those of other presidents. Furthermore, this study also assumes that this type of research will not cause a Hawthorne effect on these leaders, an effect that is defined as a "positive change in the performance of a group of persons taking part in an experiment or study due to their perception of being singled out for special consideration”(Collins English Dictionary Online, 2009). More specifically, these presidents will be asked to construct trustworthy narratives, 
absent of distortions, about what they experienced and be able to share the deepseeded meanings they gave to the situations leading to the vote. Therefore, this study assumes that these leaders will be willing to share-without being completely guarded, self-censoring or defensive - their fears or inadequacies they felt during this time, as well as missteps or mishaps they could have avoided during these votes on their leadership.

Reciprocally, this study will be assuming that the dominant master- and counter-narratives found in the literature, journal articles, and senate meeting minutes have accurately depicted the perceptions and reactions of the sponsoring constituents in text. Such texts will be aligned with the stories of these presidents to minimize the distortions in these stories. One of the ways the researcher will be able to control for these threats is by triangulating these sources and artifacts throughout the study. A final assumption in this study is that presidents are not a homogenous group and will have different types of lessons learned in their reflective process and storytelling. As a result, this study assumes that these leaders' stories will provide transferrable knowledge about their leadership experiences from which aspiring and current leaders can draw inferences. 


\section{Delimitations}

A major delimitation of this study is that it will primarily focus on the leader's voices. Therefore, the voices of the sponsors of the vote will be limited to the aforementioned literature and documents found.

\section{Definition of Terms}

\section{Sensemaking}

The definition for sensemaking is simply the process of making sense (Wick, 1995). This construct, originated in Karl Weick's (1995) work on sensemaking in organizations, has been used often in theoretical and empirical research to describe a phenomenon that only the person who experienced a situation can make sense of it. For example, in Eddy's (2005) study of nine community college presidents, used this approach to help the leaders open up about critical events (both positive and negative) in their leadership. Eddy argued that each participant found it less threatening to discuss such events, and were better able to reflect on who they were, their overall leadership, and interactions with constituents during critical events in their presidencies, when asked to make sense of it.

\section{Critical Juncture, Crisis Incidents or Crisis Events}

These terms are used interchangeably to discuss the incidents that caused or led to the no confidence votes. According to Wick (1995), these are generally the 
institutional or organizational alarms that can overshadow the role of the president, which can also disrupt the daily routines of these leaders and the organization. Such incidents, junctures or events are characterized as signals or catalysts that something went awry in certain activities such as collective bargaining negotiations or the faculty tenure process, which can cause members to try and make sense of what this shift means. In addition, such events usually come in the form of language, communications, or actions, such as emails, walk-outs, no confidence votes, or public firings, all of which are critical signals that something or someone needs the attention of the individuals or groups they are directed against (Schmidt, 2009; Smith, 2003).

\section{Structure}

According to the extant literature, structure describes both the formal ways in which the community college is organized and the informal and covert taken-forgranted ways in which they operate (Meyers \& Rowan, 2006). For example, formal structures could include the hierarchies within the organization, lines of authority between the leader and employees, or policies that govern how things will work (Birnbaum, 1988). Informal structures can include the taken-for-granted ways of operating, such as the staff, faculty and president always addressing each other by their first names. Giddens (1984) also asserted that the latter type of structure usually happens when the agents or actors within the organization give meanings to 
the formal structures and these meanings become embedded in the people who work within the organization (i.e., meanings become resources for sensemaking by members). In addition, other researchers also describe this latter informal structure as the culture or the social part of the organization that maintains the traditions and the status quo in organizations (Birnbaum, 1988; Meyers \& Rowan, 2006; Schein, 1996).

\section{Agency}

For the purpose of this study, agency is the people or groups who develop structures, work in them, and maintain them, as described by the Structuration literature (Bandura 2000; 2001,, Giddens, 1984). For example, the founding leaders in higher education designed the California Master Plan, the structure of higher education in California. This structure was constructed by elite groups of individuals who had a vision, mission and power to create these systems and give them meanings; moreover, they also worked in these systems as well. Therefore, structure and agency are coupled by a dualism concerned with structures determining the actions of people and their behaviors, as well as the agencies that work in and govern them. Meaning, this literature have shown how structure can determine agency and how agency can determine structure as well. Thus, both the structure and agency are interconnected in the organization. 


\section{Chapter Two}

\section{Introduction to Literature Search Methods}

The objective of this literature review was to delve into the literature concerning presumed consequences and aftermath of a no confidence vote crisis on the presidents in California's community colleges, by exposing the potential implications of these votes. As outlined in Chapter One, the suppositions about the rationales, implications, meanings and outcomes of a no confidence vote are limited to specific sources, such as trade journals and individual dissertations, which placed further limits on the types of analysis needed to better understand this phenomenon on leaders. Using these basic suppositions as a knowledge base, this literature considered other scholarship across different fields to further explore the potential factors, meanings and implications of these no confidence vote on presidents.

As mentioned in Chapter One, these assertions about NCVs were focused on three main rationales for the NCV resolution: a) Leaders and their style of leadership, b) Shared governance violations, and c) Communication missteps between community entities. To understand the factors that could lead to such rationales is pivotal to increasing knowledge about these votes, as well as to examine the aftermath of such votes. This review of the literature will not only provide a "structure for examining a problem" or phenomenon but it will also be used to "explore the relationships between variables" used to interpret and 
understand this no confidence vote phenomenon among presidents (Vivar, 2007, p.2).

In addition, this review included two major phases or literature searches of both primary and secondary sources, and the findings will be structured in a thematic literature review involving organizing, synthesizing, and describing literature and studies under a specific theme throughout the review (Creswell, 2007). The key concepts from this literature review will provide some insightful considerations for understanding these votes. In addition, a third section of this literature review will discuss Weick's (1995) sensemaking approach and how it is central to this study's exploration of the votes. A brief outline of this approach will cover the key approaches that leaders might use to make sense of this crisis event on their leadership.

\section{Literature Review's Research Strategies}

The initial phase of this extensive review commenced with searches in SFSU J. Paul Leonard Library Article and Database web pages for primary sources in ABI/ INFORMS Complete, ERIC, ProQuest, Google Scholar and WorldCat.org. This included reviewing a wide range of books, articles from peer-reviewed journals, and articles and newspapers, and using this knowledge to analyze and group into categorical themes. This review employed several keyword searches or descriptors to capture the most fundamental scholarly work on this issue, such as, 
but not limited to "motion of no confidence AND leadership* AND presidents*", "no confidence votes AND leadership*", or "leadership crises AND no confidence votes* AND education*", "leadership turnover AND dismissal AND succession", "shared governance AND community college*", "leadership performance AND evaluation*", "crisis AND management*", etc. Initial findings in the literature search resulted in a plethora of literature focused on leadership turnovers or crisis in leadership; however, the scholarly literature was scarce on other topics such as no confidence votes on leaders, presidents, or CEOs in leadership crisis. Nevertheless, the analysis of the literature was carried out using four major criteria: leadership crisis, gender and leadership, organizational behaviors and theories, and governance in the community college context. In addition, the literature that focused directly on this leadership phenomenon was limited to news briefs, professional papers and reports, three dissertations and one thesis found in ProQuest and other databases using WorldCat.org. These findings also prompted a second phase of reviews. The next phase of this literature review investigated news and other trade and professional journals from secondary sources, such as The Chronicle of Higher Education or Inside Higher Education, which provided a strong corpus of no confidence vote cases and analyses that were then incorporated in this review. For example, literature searches in The Chronicle of Higher Education alone resulted in over 1100 news briefs about these types of votes, and included specific incidents and situations that resulted in these votes, as well as some knowledge of causation 
and implication for these votes on the leader and organization, which were all invaluable to this review. Specific cases from these media and other sources will be referred to in this review and summarized in Appendix F.

During these two stages of the literature search, it became apparent that the evidence found in the empirical research should be used in conjunction with other sources in higher education, leadership research and organizational theories that examined the leadership and organizational antecedents and contextual variables that may be applied to, or possibly be determinants of, this phenomenon as suggested in evidence-based research (see Frederickson, Hambrick, \& Baumrin, 1988). In addition to this framework, this research selected the body of materials considered most relevant and invaluable to this study, particularly a few limited scholarly literature and dissertations that utilized a comparable methodology and analysis that mirrored that of this study. For instance, this investigation retained research that had a similar qualitative research design and engaged in the narratives of leaders, as well as those that used Karl Weick's (1995) sensemaking theory and approach for analyzing the narratives of leaders who experienced this type of crisis within their leadership.

\section{Literature Review Themes}

Using the themes developed from the final analysis of the literature and assertions about these votes from Chapter One, this body of literature identified 
areas where there were competing narratives about the factors, meanings and implications of these votes on leadership. For example, beyond the frustrations of the sponsors of the vote, some authors identified specific disagreements about the operational activities that may be the root cause for these votes, such as collective bargaining disagreements (Smith, 2003; Wright, 2007). Others posited that no confidence votes are caused by the leader's poor style of leadership that was inconsistent with the values and culture of the organization (Hernandez, 2006), or because the leader violated some shared governance tenets that led to the vote (Kuykendall, 2010; March and Weiner, 2003; Schmidt, 2009; Shulock, 2002). As previously mentioned in Chapter One, the main focus of no confidence votes is the lack of confidence institutional player may have in the leader and in his or her effectiveness in managing the overall institution, communicating effectively and as expected, meeting the needs of all stakeholders. In sum, three of the most recurrent rationales in these votes stemmed from situations related to leadership practice and style during specific work cycles, shared governance violations and communication challenges and barriers during critical events in the institution. Still, other researchers maintain that such assertions about these types of attributable factors, rationales and implications may only be skimming the surface of other undercurrent factors, such as other antecedent conditions, such as relationships with predecessors, or a history of failed strategic initiatives (Birnbaum, 1992; Shen \& Cho, 2005, Tierney, 2007). As a result, the four main rationales and themes to be 
examined include (a) leadership performance, styles and challenges; (b) contextual factors, such as shared governance, c) communication and stylistic problems, and d) other implications and consequences of no confidence votes. In addition, each of these assertions will be examined within its context and with literature across different disciplines. The next section will summarize the ways in which a no confidence vote can present a crisis on leaders and their institutions.

\section{Leadership in Crisis}

For the purpose of this study, the term crisis is being applied to these situations and is also being operationally defined here as, "a sudden and unexpected event that threatens to disrupt an organization's operations and poses both a financial and reputational threat," (Coombs, 2009, p. 164). Essentially, no confidence votes are believed to develop out of the situations leading up to the vote, such as failed collective bargaining negotiations, and the activation of the vote itself can then be the crisis event from such developments for leaders and their institutions (Schmidt, 2009; Smith, 2003). Therefore, unlike a crisis that comes as a result of a natural disaster or budget cuts against which leaders can prepare and protect their institutions, these votes may be coalesced with many other factors that leaders may or may not be cognizant of or prepared for, and these votes can thrust leaders into situations that they are not equipped to handle (Fanelli, 1997). 
Crisis management is one area that most leaders are required to have the competencies in and be able to manage. Smith and Riley (2012) argued that crisis is not something new for those in leadership, but questioned how often leaders are prepared to manage crises with "no warning...[that] emerge slowly...[last] over time" or those crisis events that last longer or faster than expected (p. 58). Meaning, researchers have asserted that during such tenuous situations, often times leaders (and their boards) were surprised by these votes against their leadership (March \& Weiner, 2003; Smith, 2003). M. Kuykendall in an interview with Schmidt (2009) further purported that leaders are often puzzled by the vote against their overall leadership, particularly during typical institutional activities, like budget cuts or negotiations. As a result, these votes may further demonstrate of the gaps and differences between how leaders are perceived and how these leaders see their leadership practice. Overall, these votes also appear to have a sudden-turn-ofevents aspect to them that can plunge these leaders into a public domain where they may be forced to defend themselves or fight to keep their current positions.

Still, it is unclear what these votes really mean for those who receive them. Although, Maitre (1999) asserted that the meaning or point of a no confidence vote is quite obvious to the leaders it targets as apparent by the actions of the leadership after the vote is a given. Perhaps, there is some validity to this claim, particularly when the sponsors of the vote call for the immediate ousting of leaders for their failed leadership style. However, M. Kuykendall in Schmidt's article (2009), Smith 
(2003) and others asserted that the reactions, consequences and outcomes of these votes are often muddled with other problems in the organization and may not be as obvious. Smith's (2003) report of no confidence votes in California's community college also supported these assertions when she noted that leaders who received these votes were often shocked and surprised by the votes, and the reactions of both these leaders and their boards to these votes can also be unpredictable. Although, the actual voices of these leaders are missing from Smith's (2003) report, it appeared that these NCVs can have unpredictable effects, such as leaders feeling personally attacked, which may not necessarily be uncommon as noted by Kiley (2012) and others. For instance, Clark College's President, R. Wayne Branch, noted that he was "caught off guard by faculty members' decision" especially because he was unclear why he was even getting the vote (Ashburn, 2006, para. 4). Essentially, the faculty gave him the vote because they believed that he had allegedly "violated his commitment to shared governance," failed in communicating with the faculty, and utilized a highly "autocratic leadership style" (Ashburn, 2006, A23). Eventually, Branch exited the institution before the end of his contract, but little is known if these votes resolved the stated issues or if the new leader's performance reduced the concerns of the faculty. The implication here is that even the actions or reactions of these leaders may not be evidence of the actual meanings of these votes. It is possible that there is more going on in these reported incidences that what is reported in the media. Therefore, understanding the 
meanings, implications and consequences of these votes is critical knowledge for this study and for both for leaders and the faculty who consider and use these sanctions against the leadership. Furthermore, the consequences of the votes may have wider implications for leaders and institutions beyond what has been previously studied.

Unpredictable outcomes. One aspect of no confidence votes (NCVs) that is explicitly clear in the literature is that their outcomes can appear nebulous. For example, there are two possible outcomes of these votes that were outlined in the literature: the leaders will either leave their institutions, or they will stay with the support of their boards (Schmidt, 2009; Smith, 2003). As a matter of fact, a major tenet of NCVs is that they often call for the immediate ousting of leaders as the desired outcome of the vote. As previously mentioned in Chapter One, these votes do not have the formal authority to automatically depose a leader from his or her institution (Tierney, 2007); albeit, they do appear to at least color the opinions of others about the leader and amass the attention needed about certain problems in the institution. As a matter of fact, boards still retain the legal responsibility for appointing and dismissing their presidents, thus rendering these votes ineffective in automatically removing a leader like the British process (American Association of University Professors (AAUP, 2005). As a matter of fact, the AAUP (2005) afforded each college the right to evaluate, "be sharply critical of... an 
administrator's performance... when a faculty senate passes a vote of no confidence" (AAUP, 2005). For example, in the case of President Jerry Sue Thornton at Cuyahoga Community College, she not only had no intentions of leaving her institution after the vote, but her contract was renewed and she had strong commitment from the board (Farkas, 2010). President Thornton received a previous vote in 2007 from the faculty union for alleged poor leadership abilities (Wright, 2007), and the more recent vote was given on the heels of failed negotiations from the faculty union (Farkas, 2010).

The unpredictability of NCVs can also be seen in the case of President Linda Katehi at the University of California, Davis. President Katehi was targeted for the vote in 2012 after police excessively doused students with pepper spray directly in their faces during a peaceful protest, and after this incident was broadcasted in news cycles on national television (Chea, 2012; Gordon, 2012). Although President Katehi was believed to have no direct involvement in the incident other than authorizing the police to remove the tents of protester without pepper spray, some of the faculty drew attention to the incident and held her responsible for its occurrence during their calls for a vote (Gordon, 2012). For this leader, the motion was raised and carried forward but was unsuccessful because more faculty voted against the motion than for it. As a matter of fact, the faculties called for a second vote to demonstrate their confidence in President Katehi's leadership (Gordon, 2012). 
A major argument concerning the inconsistencies of the outcomes of these votes may be influenced by the mindsets about what these votes actually mean. Specifically, a common argument about NCVs is that they are essentially arbitrary with innocuous qualities because they cannot legally remove leaders from their positions, and because of their apparent overuse in certain institutions that could render them an ineffective option overtime (Wright, 2007). While some view these votes are merely a means to an end that should not be taken so seriously because these actions are symbolic in nature and signal to the larger community to take notice of whatever is going on (Schmidt, 2009; Shaw, 2005). Other authors suggested that this resolution is arbitrary but has too many ambiguous processes and consequences, especially since one cannot guarantee that the board will pay attention or respond to them (for example, see Tierney, 2007). In addition, this concept about the arbitrariness of the votes can also have implication on how institutional players use this mechanism and how leaders and their boards can grow accustomed to the constant bellyaching of the sponsoring group that they may begin to ignore these votes and view them as benign (Wright, 2007). The assertions here is that these votes may be less than effective and credible as some believe.

No confidence votes may also be viewed as a means for institutional players to voice concerns about the leadership, whether or not necessarily such concerns are reflective of the overall performance of the president (e.g. March \& Weiner, 2003). As a result, these votes as also viewed as having such a neutralizing effect that any 
attempts to understand them may be futile (Monaghan, 1998). A major inference here is that because these votes may have such non-threatening effects, there should not be any serious investigation and scholarship about them, which this researcher profoundly disagrees with seeing that there are so many unknowns. These examples and assertions further demonstrate that overall, the outcomes and consequences of a no confidence vote appear to vary and may be highly unpredictable when enacted within the climates of these institutions. In most cases researched, this climate was also highly dependent on the board's response of the vote. Therefore, if boards publicly stood steadfast in their support for their presidents, for example, such presidents usually stayed in their institutions (Schmidt, 2009; 2010 and Tierney, 2007). Otherwise, a lack of support from the board for any given reason, including the vote, can end these leader's tenures. As a result, the outcomes of these no confidence votes are often unpredictable, and they may or may not have the expected outcomes as intended by the sponsoring group against the leadership.

Under-regulated process. Another characteristic of the vote is that any number of constituents can raise the vote against the leadership; allowing each institution the power to regulate this process for their purposes. More specifically, no comprehensive federal, state or district regulations were found concerning when and how a no confidence vote should be raised. For example, in most reports examined, it appeared that, based on the institutions voting policies, any qualified number of faculty may choose to go through their academic senates to raise the vote 
against the leadership, not necessarily the standard two-thirds (Maitre, 1999; Trivedi, 2011). Likewise, without specific regulations any constituency group, such as unions, students, or individual departments in the institution for example, can raise a NCV motion with or without going through a senate process or any type of regulated voting process. Virtually unrestricted, it does appear that most institutions have the autonomy to determine how and when they will utilize and enforce no confidence votes in their organizations, which can still be a highly effective process for getting the attention of these leaders and their boards (Tierney, 2007).

Similarly, the process for managing the aftermath of a NCV also appears to be less than transparent or consistent. This study was able to locate only one school system with policies regarding what should happen after these motions are raised. As a matter of fact, none of the reports researched detailed their regulated processes and policies for raising the motion, and most of these reports focused on the aftermath of the vote. For example, once the decision is made to pass this motion and a vote is enacted against the leadership, the sponsors of the vote are generally required to present a list of grievances, also known as a bill of particulars, to the individuals targeted and their corresponding administrators, such as the board of trustees or a chancellor. After which, the board or responsible administrator reviews these concerns and demands to determine whether or not they will continue supporting the leader and/or respond to the concerns of the sponsors directly, with 
the targeted individual(s) or with the assistance of consultants (Kuykendall, 2010; Schmidt, 2009).

Interestingly, in the California community college system, one of the only clearly outlined regulations found was a Senate Bill (SB) 55-Lowenthal (Senate Bill, 2005). Senate Bill 55 was ratified in 2005 and required boards to address the concerns raised by all internal and external stakeholders, including constituents who sponsor the vote, through a series of meetings with the faculty or sponsors of the vote, as well as to investigate the concerns outlined in the bill of particulars (Senate Bill, 2005). This particular system has had a history of some boards refusing to acknowledge or even respond to the resolution when passed (Smith, 2003). It is unclear why this college system and other institutions would have definitively outlined regulations for only the tail-end portion of this process as opposed to a regulation before the vote. Additionally, it is possible that these votes have remained unregulated because they do not have any formal power to dismiss leaders or presidents in the institutions. However, without such regulations, it is unclear how different organizations attach meanings, institutionalize these sanctions in their organizations, or survive the potential consequences of the vote (Schmidt, 2009; Smith, 2003; Tierney, 2007).

Small groups of voters may have big consequences. A third characteristic of no confidence votes is that they appear to represent a fraction of the faculty or other constituency groups, who are often times a small number of voters in proportion to 
the total number of faculty or personnel (Schmidt, 2009; Kuykendall, 2010). M. Kuykendall in an interview with Schmidt (2009) further posited that the size of the organization matters and notes that smaller numbers of voters are common in larger and more decentralized systems as compared to smaller and more centralized institutions. For example, a single department may enact a vote, as seen at the University of Missouri with twenty-six members of the English department giving the vote (Vandelinder, 2015). Without specific policies to govern these votes, it appears that any number of individuals can raise these votes without going through a formal process, as seen with an independent adjunct faculty union group who wielded the vote against the president and board at Columbia College, because of deeply contested changes to the format of a first year experience seminar without their alleged inputs (Inside Higher Education, 2015). Altogether, a major criticism of these no confidence reports is that they appear controversial in that they often present a collective mindset of the institution against the leadership but fail to mention the institution's regulated processes or the exact number of qualified voters who were involved in the process, to at least statistically illustrate the extent of the problems. Nevertheless, the apparent main emphasis in these reports, and in the media, is in presenting a unified or coalesced view of the sponsoring group's frustrations and concerns within the organization and against the leadership, whether or not their institution's guidelines about quorum or regulations were properly followed (for examples, see Fain, 2007; Gordon, 2012). 
Smerek (2011) described such collective actions as a type of sensegiving process, which is a process used to influence others either positively or negatively about situations going on in their institutions. For example, one writer described no confidence votes as both symbolic act and a means for the "tenured to bully the untenured" (Dad, 2008, para. 10). However, from the cases reviewed, it is not easy to determine if there were such hidden agendas involved with these votes other than what was stated in these reports. Neither is clear the extent to which these votes are being used, beyond gaining the attention of the president and board. Such suppositions can only be confirmed by individuals who have experienced these votes in their institutions. Thus, a final consideration about these highly publicized reports is that they appear to represent this collective mindset against the leadership, and it is unclear how these reports can also influence the perceptions of the general public and other stakeholders, particularly when these tensions are publicized in the media (The Economist, 2009).

Potential costs associated with no confidence votes. Findings in the literature review also discussed the possible unforeseen costs for replacing these leaders. The presumable costs associated with dismissals, included costs associated with unfulfilled contracts and replacing the leader, for example (Nalbantian, 2012). According to Smith (2003), in the California community college system, "one third" of the leaders who received the vote actually left the presidency and would end their presidential tenures "within a year" after the vote (p. 6). It is unclear, 
however, if these leaders left because of the vote or because of other factors. Trombley (1997) speculated that some of these leaders may have left their organizations because of other factors or opportunities available in their personal lives and some leaders may have decided to simply buy out their contracts and leave their organizations. As Smith (2003) noted, the reasons these leaders leave soon after the vote is usually varied, and that many of these leaders do not necessarily suggest that the vote was a factor in their decisions.

From these reports, it is unclear what the impact of these leaders' departures could mean for boards and for constituents. As speculated in Chapter One, perhaps certain constituents feel relieved when the cause of the problems in the institutions is finally leaving. Still, it is unclear if boards are further impacted when these leaders leave abruptly, and whether or not they had a succession plan in place, beyond the typical use of interim leaders. For instance, with strained relationships within the organization and dwindling budgets, it is unclear if boards understand the costly impact of replacing these leaders. Turnage (2015) further posited that although the departures of leaders could be the result of several factors, such as mergers, retirements and firing, that these leaders' departures can have hidden costs, particularly when it's unexpected. Forbes magazine reported that the cost of "unplanned or sudden" ousting of leaders could cost large organizations as much as "\$1.8million in share holder value", particularly when boards are not prioritizing the process of exit and succession well (Favaro, Karlsson \&Neilson, 2015, para. 2). 
Although this cost to institutions in higher education may be significantly less for community college or universities, this pattern of habitually replacing leaders can be a costly cycle for boards and their institutions. This study also hypothesizes that there are possible ramifications to these types of leadership exits, and even with excellent succession plans, the process could still be costly one for any organization. Table 4 further illustrates the possible costs associated with leaders leaving their institutions as a result of turnovers for any reason.

Table 4

Potential Costs Associated with the Unexpected Ousting of Leaders

\begin{tabular}{|c|c|}
\hline Cost of Hiring After No Confidence Vote (assumptions) & $\begin{array}{l}\text { Costs (increases with timeline to } \\
\text { secure a president) }\end{array}$ \\
\hline Hiring a third party agency to recruit candidates for the position & $\begin{array}{l}\$ 200,000 \text { (per year minimum } \\
\text { contract) }\end{array}$ \\
\hline Secure interim-president \& search process & $\$ 100,000$ (approximations) \\
\hline $\begin{array}{l}\text { Cost of relocation for the leader and family (not including } \\
\text { individuals who will need special accommodations due to a } \\
\text { disability). }\end{array}$ & $\$ 10,000$ (approximately) \\
\hline $\begin{array}{l}\text { New position includes a salary increase for incumbent. } \\
\text { According to Nalbantian }(2012) \text { in some cases as much as } 30 \% \\
\text { more than the previous leader. }\end{array}$ & $\begin{array}{l}\text { An additional } \$ 80-\$ 100,000 \\
\text { added to the base salary. }\end{array}$ \\
\hline TOTALS & $\begin{array}{l}\$ 390,000-\$ 400,000 \text { of incurred } \\
\text { costs associated with hiring a } \\
\text { president }\end{array}$ \\
\hline
\end{tabular}

Note: This table was adopted from Kim (2011) and Nalbantian (2012). 
Overall, as presidents leave their institutions before the end of their contracts, board may scramble to fill the position on an interim basis with someone from within the organization, such as a provost or vice president. However, the process of replacing and hiring a new president can be quite expensive, as illustrated in Table 4. Kim (2011) inferred that, on average, most public college and university boards, of course using state funds, may spend-at a minimum$\$ 200,000$ to simply secure a private hiring agency to attract and recruit leaders for the organization. Nalbantian's (2012) argument further supported Kim's assertions, when he claimed that the overall presidential campaign is not only very expensive but some can persist beyond six months and can last as long as two to three years, which can increase these costs. Such costs may not only be devastating for organizations already stretching their budgets to survive in a recession, but they can be the catalyst for other crises in the organization that disrupt how resources are allocated and what work takes priority in these processes.

\section{Impact of a no confidence vote on the leadership and institution. A} major consequence of these NCVs is related to the possible impact it can have on these leaders and their reputations, during they tenures in these institutions and after they leave (March and Weiner, 2003). These writers and others contended that constituency groups who use that these votes to actively oppose the leadership, may do so after all other efforts to resolve their concerns were tried and were presumably ineffective (Petrick, 2007). Other authors asserted that the meaning of 
such public displays of friction stems from a history of power and political posturing between faculty and administration (Birnbaum, 1992; Schmidt, 2009; Wilson, Fain, Fogg, Selingo, 2006), further implying that this practice has some level of normalcy. Borrowing from the literature on leadership tenures, Coyne and Coyne (2007) advised of the leaders who were dismissed or left their organizations under some type of shadow, only a few of them were able to pursue similar high level positions in their careers; further highlighting some of the potential consequences on leaders and their careers. The consequences of these votes on the reputations of the leaders also appear to be highly correlated with the reactions of their boards (Schmidt, 2009). For example, there are authors who have suggested that some boards react by showing their strong support of the president by giving them raises or extending their contracts, and that such actions act like a type of slap in the face to the sponsoring group (for example, see Wright, 2007).

Still others regard these votes as an effective tool for institutional players to publicly communicate their concerns and criticisms about the administration, especially when leaders leave (Kuykendall, 2010; Schmidt, 2009). However, there are others researchers who caution against such actions and suggest that the impact of the vote can have an impact on the reputations of the institutions, such as setting precedence about the expectations, politics and cultures within the organization (Tierney, 2007). Kiley (2013) also advised that NCVs used against the leaders can be viewed as a means to publicly "shift opinions" about the leadership and his or 
her performance to both the internal constituents and external stakeholders (Kiley, 2013). Such suppositions further implied that perhaps these votes may be accompanied by something more sinister impact on these organization's reputations (Tierney, 2007). For example, one former State Academic Senate President further posited that in some institutions the vituperative actions and steps used by institutional players to enact a vote against the leader can be likened to a form of herding or mob mentality, rather than a last resort to oust the leader (StanbackStroud, personal communication, May 20, 2013). A herd or mob mentality refers to the ways in which individuals can influence the thinking of their respective groups to mobilize and act upon that thinking, oftentimes as a kneejerk reaction out of anger, for instance (McCauley, 1989).

There appears to be dual implications for both leaders and their institutions, and that neither entity is unscathed by these situations. Ith (2002) explained that the consequences of the votes are so unstable that they can either "backfire" against the sponsoring group, for example, as well as it is something that can be "survived" (para. 1). One example of how this can backfire against the sponsoring group is that some boards have moved to dissolve their faculty senates and governance structures as a type of retaliation against the sponsoring group for the vote against the president, as seen in the case at Texas A\&M University at Kingsville (Olson, 2011). For this reason, a few authors have cautioned constituents against using such an "indictment" or "serious criticism of the president" because they may have potential 
repercussions for the sponsoring group (MacTaggart, 2012, para. 16 \& 19). For one researcher, Tierney (2007), he further posited that both the faculty and other groups should avoid this "nuclear option" and for leaders to take steps to "avert" it (Tierney, 2007, para. 1-2). He further cautioned those who would consider this option, even as a last resort, primarily because of its disruptive nature and potential rift that could deepen between the two groups. Other authors further implied that a no confidence vote option, particularly when used often, could not only taint the reputations of the leaders in these institutions, but it could lower morale and eventually damage the reputation of the institution as a challenging work climate for future potential leadership candidates and other stakeholders (Farkas, 2013; McGrath, 2015; Song, 2014). Regardless of the suspected intentions for using these votes proposed by these writers, little data were available to support such suppositions to determine if such actions could undermine the reputations of these institutions or that their leaders, a key area that this study hopes to address.

In summary, the crisis of a no confidence vote are often dependent on many complex factors and may not result in the intended outcomes or have the desired impact on leaders or their context-specific institutions. As a matter of fact, the consequences of the vote may have serious implications for both these leaders, as well as the sponsors of the vote. With the outcomes of these votes being unpredictable, unregulated, potentially costly, and damaging to the reputations of these leaders and their institutions, more research is needed to unpack the duality 
found in these concepts about NCVs in higher education. Overall, these NCV crisis events are dependent on various contextual factors, such as shared governance violations; emerge from out of the perceptions and interactions with the leadership, and may have many unforeseen implications on both the leadership and the overall organization. The next section of this review will delve into the no confidence vote major themes found in the literature.

\section{Theme One: Leadership Performance and Challenges associated with NCVs}

A pointed criticism in most no confidence votes is against the leader, who institutional player may label as being an overall ineffective leader (Schmidt, 2009). Smith (2003) asserted that up to a third of the no confidence votes she analyzed in California's community colleges were given because sponsors said they either lacked confidence in the style of the leader or in his/ her ability to lead the institution effectively. As outlined in Chapter One, in most of the cases reviewed, evidence of such accusations of failed leadership were hardly substantiated in reports, neither were copies of performance evaluations or specific assessments offered beyond assertions that leaders may have violated some expectations about how they will perform during specific cycles of work, such as shared governance initiatives, budget planning, etc. This does not mean that the accusations of these institutional players against the leader were completely baseless or that these 
leaders were not ineffectual. However, these reports do provide very little evidence about what may have caused such criticisms to emerge against the leadership.

M. Kuykendall in an interview with Schmidt (2009) also found that sometimes these NCV rationales about ineffective leadership were ambiguous and the leader's responses somewhat scripted, which was also a common thread across these vote. In addition, she also found conflicting rhetoric among leaders about what these vote for ineffective leadership actually means. For instance, $M$. Kuykendall also found that leaders were more than likely to claim that they didn't get the vote because of ineffective leadership, but they got these votes because institutional players were opposed to them challenging the status quo or trying to implement changes, as opposed to some type of professional or "personal flaw" (Schmidt, 2009, para. 3). Beyond being entangled with other issues within the organization, NCVs that were given on account of presidents being ineffective leaders is being taken seriously and demands further investigations. Therefore, this literature review section deconstructed some of the problems associated with leadership in the academy, and the factors that might be contributing to these votes as key concepts to be explored and used to better understand the rationales surrounding a lack of confidence in the leadership.

Leadership performance and effectiveness. The role of presidents in the academy has been described as one of the most critical and complex roles in higher 
education (Birnbaum, 1992) for three reasons. 1) The conditions within the community college environment are continuously changing and shifting to meet the many missions and needs of its environment (Brint \& Karabel, 1989; Shulock, 2002). 2) Some see the environment as highly complex with hierarchies and structures or systems of controls that can support or constrain leadership (Kirkland \& Ratcliff, 1994). 3) Others see the role of leaders as incessantly shifting to survive and limit the risks to their jobs (Eddy \& VanDerLinden, 2006). In light of these complex systems, there are decades of literature that has been informing society about leadership and what effective leadership is in different environments.

The concept of leadership has been one of the most studied topics with a myriad of theories used to inform and permeated society's ability to identify leadership, leadership theories and models, and leadership behaviors in almost every area of our society (Horner, 1997; Sinclair, 1998). Perhaps, society is in search of the ideal model of leadership for specific situations, but what we are certainly left with is some idea of what leadership is, what is should look like and what it is not. For example, these pluralistic leadership concepts and frameworks range from promoting traditional leadership traits, such as being assertive and intelligent (Burns, 1978; Gardner \& Hatch, 1989); styles, such as being autocratic or democratic (Lewin, Lippitt \& White, 1939), to advocating for certain leadership practices and approaches deemed most effective, such as transformational (Bass, 1985; Burns, 1978) and transactional (Bass, 1990), instructional (Hallinger, 2003), 
shared (Lambert, 2002), and distributed leadership to lead groups and coalition of groups in most organizations (Brooks, Hodgins, Jean-Marie \& Normore, 2007; Hargreaves \& Fink, 2008; Spillane, Diamond \& Halverson, 2004). Essentially, these discussions about leadership and what is or should be have rendered a glut of literature that have been used to identity, socialize and define what leadership is or should be in the mindsets of society, including institutional players within higher education.

Leaders in California's community colleges are expected to possess and demonstrate certain skills and competencies such as having a vision or skills to lead their organizations (Bass, 1990; Birnbaum, 1990), particularly when such leaders need to manage crisis events and high-risk situations (Boin \& Fishbacher-Smith, 2011; Smith \& Riley, 2012; Wooten \& James, 2008). The literature on competencies also tends to focus on how these leaders were or were not able to move the organization forward and develop policies to safeguard or respond to critical events in their organizations, such as campus shootings or on-campus sexual assault (Boin, Hart, Stern, \& Sundellius, 2005). This research found a dearth of scholarship that discussed a crisis facing the leader or when the leader is the actual person in a crisis, how they make sense of it (Gioia \& Chittipedia, 1991), and the impact the media can have in "shaping...post-crisis accounts" and maintaining certain shared meanings after certain crisis events (Maitlis \& Sonenshein, 2010, p. 557 and 561). Although most no confidence votes are often reported in less formal 
media, such as trade journals and national papers, the circulations of these reports can still have an impactful role in influencing the community's awareness about what is going on during turbulent times. In addition, these reports also fail to present any follow-up reports or to provide any divergent views about these crisis incidents (Maitlis \& Sonenshein, 2010).

Generally, the literatures found on leadership competencies also appeared to focus on leadership skills and development. For example, Bennis' (2004) work on the seven ages of a leader identified the leader's development, including the infant executive, schoolboy, lover, soldier, general, statesman, and sage levels of expertise. He believed that it is through these stages of development that the leader will acquire certain skills, strategies, and experiences that allow them to become more comfortable in his or her role. Bennis' (2004) theory delineated the ways in which leaders may identify their own brand of leadership, how others may perceive and respond to these leaders during each age of development, and how these expectations, both covert and overt, can influence and impact these leaders. For example, he asserted that leaders generally go through these stages every time they enter a new organization and they tend to carry over knowledge from their previous experiences that may or may not apply to their new environment (Bennis, 2004). As a result, he cautioned leaders to sharpen their leadership skills and focus during these stages of development. Meaning, during the infant and schoolboy ages of leadership, he maintains that leaders must be cognizant that they are entering a new 
structure or environment with its own rules of doing and being that they will need to learn. Similarly, during the second age, Bennis (2004) also asserted that leaders may find that their leadership is being defined by the perceptions of others and their evaluations of these leaders' actions and decisions, whether these views are accurate or not. By the third age, the lover, Bennis (2004) found that leaders must begin to address a myriad of problems, emotions, and concerns, and these leaders must demonstrate that they have the competencies and confidence to tackle these challenges effectively and are able to mobilize their organization. He further posited that it is during the fourth age that leaders may find support and resistance about their approach or style to leadership, and are also able to assess their limitations and areas of expertise in their role. By the fifth and sixth ages of leadership Bennis (2004) found that leaders should be able to recognize and acknowledge the blind spots in their leadership, grow in expertise, and be open to different interpretation of their leadership, as they continue to learn more about the history and context of the organization they work in. By the final stage, the sage, leaders may then look back to pull up others to succeed them in their leadership role. The author noted that leaders at this age are willing to develop relationships that will allow them to share their knowledge and experiences with aspiring leaders, and to continue their legacy in the organization. As a result and over time, leaders may have acquired certain skills, strategies, and experiences that allow them to become more comfortable in their roles. 
Similarly, Dreyfus (2006) proposed a five-stage model for leadership skill acquisition, wherein leaders may develop from novice to expert leader. These stages appear to overlap with the ages of leadership development found in Bennis' (2004) work. His leadership stages progresses from the novice, advance beginner, competent, proficient to the expert leader. For example, Dreyfus (2006) explains that the novice leader is generally focused on following rigid rules and plans, without discretion or judgment as he or she develops the skills to perform responsibilities. While the expert leader is one who exceeds reliance on rules, or guidelines, and maxims. This person has an excellent grasp of situations based on understanding of the situation and has a vision as well as multiple approaches for what's possible, both for old and new situations or problems that arise.

A major criticism of both Bennis (2004) and Dreyfus' (2006) leadership development framework is the assumption that leaders will somehow naturally develop these skills, if given the time to do so. Neither of these frameworks provided a timeline for such skill development or addressed the factors that could limit such leadership competencies and developments, such as a no confidence vote. In addition, these authors often used very masculine undertones to describe the competencies or skills acquisition leaders needed to effectively lead organizations, which is not surprising since a significant portion of the leadership literature is focused on gendered ways of leading, which by itself can be a factor in what individuals consider effective leadership. For California's community colleges, 
leaders within this system are usually heavily vetted by their system, and many of them have served as presidents or an executive in other institutions, prior to getting the vote (Mize, 2011), therefore, it is unclear what competencies or skills these leaders failed to possess to make them an ineffective leader overall. This is not to say that these leaders didn't have flaws or may have failed in their leadership prior to these votes, but these types of leadership development literature often times do not address other determinants that could affect one's leadership. Still, the literature on leadership performance and competence provided a baseline about the perceptions and expectations of how leaders will perform in their institutions.

Perceptions about leaders within specific contexts. Beyond the mainstream narratives about effective leadership, are the literatures that discussed leadership within a specific context and how the leader's authority and work can be supported or constrained by these environments (Birnbaum, 1998). As previously noted, the community colleges context can be very challenging for leaders, which some have described as "social arrangements of communities of practice and networks," and a very structured and highly complex system (Hargreaves \& Fink, 2008, p. 229). Hargreaves and Fink (2008) described this space, wherein leaders must work and make sense of, as a structure and system of "command and control within loosely and tightly coupled structures", which pertains to the types of interactions leaders and constituents will have in these environments (pp. 229-230). 
It is within this space where leaders are expected to perform their work well and are required to use close maneuverings, negotiations, and trust across different groups in the institution to be successful (Herbet-Swartzer \& McNair, 2010). The inferences from these authors is that leaders need to understand the context in which they work well, and that they may be perceived differently across different departments, divisions, communities in and around a singular institution as they interact with institutional players, and they may also be judged differently by various stakeholders and affiliates both within and outside the institution.

Structurally, the community college system is a system with pluralistic structural models that leaders must learn how to oversee and lead in (Birnbaum, 1988), with multiple layers and divisions of labor with power differentiations. Birnbaum's (1987) description of colleges comes out of one of his most seminal longitudinal studies with presidents from 32 institutions over a five year period. In addition to highlighting the different leadership theories and practices of leadership that leaders ascribed to, Birnbaum (1988) uncovered some of the contextual constraints and barriers to their leadership, which were often politically charged and culturally focused contexts that leaders must work within. He then described this structure as cybernetic, a system with more than one structure within in, which could include hierarchical, loosely coupled, decentralized and centralized structures that can exist simultaneously in a single institution, and a system wherein leaders need to understand and build their own "locus of influence" or sphere of influence 
between all constituency groups and stakeholders both locally and statewide (Birnbaum, 1988, p.17). Birnbaum (1988) described this sphere of influence as the space where leaders understand the power they have to lead and to coordinate work within the institution, as well as recognize the constraints, which can shape perceptions about their leadership. He described this power as "ability to produce intended change in others, to influence them so that they will be more likely to act in accordance with one's own preferences" (p.12). However, borrowing from Karl Weick's (1976) work on tight and loosely coupled systems in education, Birnbaum (1988) cautioned leaders to understand that the structure of community college are mostly a system made up of tightly and loosely coupled systems, across the division of labor. He also proposed that tightly coupled systems require individuals or groups to work closely together and when one area within that system changes it affects the others (Birnbaum 1988).

Birnbaum's (1988) assessment of this context and how leaders must understand their specific institutional contexts and manage perceptions and their power to be effective can also be applied to leaders in community colleges in California. Gupta, Dirsmith, Fogarty (1994) discussed the overt and covert uses of power, influence, and policy in a federal auditing agency and how these elements can shape perceptions about leadership effectiveness as leaders coordinate work in these highly bureaucratic environments. Gupta et al. (1994) found that auditors who preferred rules and policy focused supervisors were more satisfied with leaders who 
built their practice around these elements to coordinate the work and teams. Essentially, these writers are suggesting that a leader's effectiveness may be dependent on how they are able to coordinate their work and support their work of others in these highly structured environments.

Birnbaum (1988) also described the community college environment as structures that are tightly coupled together and loosely coupled as well. For example, the faculty in an English department can be considered tightly coupled together for two main reasons: they work in close proximity to each other where the benefits and problems within that unit are better contained, as well as they share certain technical expertise, similar identities, and experiences together (Birnbaum, 1988). The inference here is that leaders in colleges may be expected to be someone who will participate, support and collaborate closely with these types of units within the organization. Secondly, Birnbaum (1988) also described this system as being loosely coupled together, a structural design used to minimize certain impacts in the larger institution, and a space wherein individuals and groups work independently and more specialized than other groups. Therefore, the English department may be tightly coupled within that unit but they are loosely coupled to the Chemistry department. Therefore, events local to the English department may have very limited impact on the Chemistry department, because these groups may not be routinely interacting with the other in this context. Similarly, Elmore (2004) described loosely coupled systems as having a goal of protecting the core of 
education, such as the teaching and learning in the classroom, maintaining the stability and status quo of such as system, and buffering against outside forces that require accountability with the help of leaders, for example.

Birnbaum (1988) cautioned leaders to be cognizant of these dual structures within their organizations and to understand that such structures can be difficult to predict and perceptions harder to manage. For instance, he points out that in loosely coupled systems it may be "difficult to get things done" because it is harder to communicate effectively or effect change successfully across all groups and divisions of labor (p. 40). Birnbaum (1988) also suggested that these groups begin to build perceptions as well as change perceptions about the leader over time, and that such perceptions may be based on the ways they perceive or make sense of the leader's behaviors and interactions with them. Other scholars have also described the structure as a system of hierarchies of "command and control within loosely and tightly coupled structures" within the organization (Hargreaves \& Fink, 2008, pp. 229-230).

\section{Perceptions about leadership effectiveness and practice in community}

colleges. The complexities within the community college system may be better understood in the ways leaders are expected to operate inside of it. As a matter of fact, Healey (1997) referred to the structures within this system as a "schizophrenic system," because it is top heavy with local controls and elected officials in a "highly political culture". Healy (997) also suggested that those who attempted to lead in it 
must be very responsive to both of these groups and those who parcel out funding (p. A33). For example, in the CCC system, college presidents are required to respond to several lines of authority that can be hierarchical and horizontal, such as the state and legislative charters, a board of governors, state academic senates, board of trustees, and district boards in their communities, in addition to working within a shared governance system (Shulock, 2002). In addition to being a cosmopolitan leader who takes care of the external communities, leaders are expected to remain local and highly responsive to both external and internal constituents within the organization (Birnbaum, 1988).

Kezar (1998) also found that even with such collaborative models and highly competent leadership the perceptions that faculty may have about a leader may not be different from their personal and traditional understandings about what is effective leadership. From her case study with over 30 faculty and administrators in community colleges, Kezar (1998) found that both leaders and faculty may have misaligned perceptions about leadership and its effectiveness. Kezar (1998) posited that the faculty's institutional values, mindsets, and communication preferences were often divergent from those of the administrators which may have caused misalignment and conflict during several situations, and affected their perceptions about leadership. As a result, leaders in this system may be required to demonstrate certain social capitals and competencies to lead effectively in these institutions, while managing the perceptions of institutional players. 
California's community college structure also requires leaders to be knowledgeable and responsive to an insurmountable amount of college charters, laws, and policies that will help to support them in this complex system. For example, like all public education systems, the California community college system adheres to certain state laws and policies about how the work of the college is to be managed. For example, leaders are expected to adhere to the shared governance mandate AB 1725, which Shulock (2002) and others have asserted may be more difficult to realize on the actual college campuses, and can result in increased tensions between a leader and constituents (Collins, 2002; O'Banion, 2010; Olson, 2009; Tierney, 2007). O'Banion (2010) suggested that this reform was developed to forge a new path for community colleges within California and to create "a new model for decision making" between the administration and faculty (pp.191-192).

With this new law came changes that some authors asserted some leaders were often ill prepared for, and would may have lead to several areas of contention between these leaders and constituents as they tried to adopt to this coalesced governance model for working together (Collins, 2002; Olson, 2009). Anecdotally, leaders whose leaderships appeared out of step with such statutes, may be viewed as violating such policy and may risk being evaluated as an unfit leader, incapable of meeting the needs of his/her stakeholders. Essentially, Shulock (2002) proposed that the California community college system is still evolving and in itself still in 
need of some fine tuning, especially in the hearts of both the leader and the faculty as they find ways to work together.

In addition to the policies that provided presidents with guidelines as to what is expected in California's community college system, there were also guidelines that identified the skills and competencies that leaders needed to perform effectively. For instance, in 2005, the California system institutionalized the American Association of Community College Core Competencies for Leadership, which identified six core skills-sets that leaders must use to perform their responsibilities (McNair, 2010): organizational strategies, resource management, communication, collaboration, community college advocacy, and professionalism.

McNair (2010) completed a study to gather data about current presidents and whether or not they had mastered these competencies, how they learned or mastered them, and whether or not these competencies were essential for them to do their work. Although the researcher received less than 10\% (113) response rate $(n=1073)$ nationwide, these findings indicated that all participants, presidents, vicepresidents, senate presidents, and chief academic officers strongly agreed that the six core competencies were essential for their jobs, with communication being the most important. As to where these leaders gained their knowledge for the competencies, in ranked order, most leaders claimed they learned them during (a) on the job training, (b) professional development, and (c) mentoring. The researcher also determined that doctoral programs were the least effective method 
for preparing leaders for leadership in their organizations. All participants indicated that they did not feel they had a mastery of these competencies and that they were always working to improve themselves and their leadership practice.

Overall, Lambert (2002), posited that in order for current leaders to perform successful they would need to expand their thinking beyond adopting a singular framework, and be espoused to leading with multiple traits, styles and frameworks that best fit their situations. The result of such literature and concepts about effective leadership may not only be deterministic and often times too rationale (Spillane et al., 2004), but they often shaded the lenses of those who observed such leaders, such as constituents and stakeholders. In addition, Horner's (1997) analysis of large corpus of leadership and management literature showed that such traditional concepts about leadership continue to proliferate over time, primarily as a result of the frameworks most leaders still adopt to define their leadership practice and style. The end result of decades of leadership research is that leaders who work in team like environments that require buy-in and vetting are encouraged the demonstrate the competencies needed for effective leadership and to use of several approaches to leadership to build trust and shape perceptions through interactions with institutional players about their leadership and their work (Horner, 1997; Otara, 2011). 
Leadership styles or approaches. One of the major factors surrounding NCVs were concerns about the leader's style of leadership as being ineffective (Schmidt, 2009; Smith, 2003). The organizational theory and behaviors literature suggested that the practice of leadership or leadership style is concerned with how leaders use all types of powers available to them, including positional and personal power, and organizational politics, to influence and empower interdependent groups and coalitions to participate in a collective effort to get something done (Schermerhorn et al., 2012). There is some agreement on the parts of members that leaders must consistently evaluate and incorporate different styles and approaches, such as participatory or transformational, and use all types of powers, which may also be dependent on the situations and individuals they are attempting to lead (Hersey \& Blanchard, 1988; Schermerhorn et al., 2012). In addition, other scholars have suggested that leadership in the academy is not about using a top-down approach; although this environment is hierarchical, it is "an interactive process that involves the CEO, the college culture, and the environment" and about leaders managing relationships with constituents (Kirkland \& Ratcliff, 1994, p. 10). A major inference from this literature is that leaders who fail to maintain strong relationships with institutional players, particularly through several avenues of communication, may find that their leadership and leadership actions can be easily misunderstood (Schmidt, 2009). 
For instance, in the case of Shirley Jackson at Rensselaer Polytechnic Institute, the faculty voted " 155 to 149 in her favor" causing the vote to fail, but the sentiments about her overall leadership style persisted (Jaschik, 2006, para, 3). Jackson, who in her first two years was praised for bringing in $\$ 360$ million to the university and raising faculty salaries by $16 \%$ in four years, was threatened with the vote after allegedly filling administrative positions without consulting with the faculty (Campbell \& Koretz, 2010). However, other antecedent conditions during this time included what some viewed as unfair employee and budget cuts, coupled with a failure to communicate with constituents (Williams June, 2007). Nonetheless, the board was supportive of her, and not only sided with Jackson, but gave her an undisclosed raise (Wasley, 2007). More significantly, the university's Board retaliated against the faculty and "disband[ed] the university's Faculty Senate...stating that it lost credibility," especially after voting in a senate president who pushed the no confidence vote on Shirley Jackson (Wasley, 2007). The only narrative captured from this president about the vote, was that she "felt some degree of angst" throughout the process (Williams June, 2007, A24). In such scenarios, the leaders who decided to stay, even for a year or more after the vote, were highly supported by their Boards, who have the sole authority to hire or fire presidents. Even with such support, leaders may still be impacted by these votes, which can lead all sorts of outcomes and constraints on their leadership (Schmidt, 2009). 
Understanding how highly complex systems like community colleges can either support or constrain the leader's capacity to lead or misunderstand their ways of leading is vital to this study (Birnbaum, 1988). Souba, Mauger, Day (2007) conducted a mixed-methods study to assess what faculty and deans valued in their leaders, such as department chairs, within 125 medical schools. The result indicated that the participants (faculty and deans) had espoused core values that contributed to their perceptions of leadership and eventually placed constraints on how leaders (department chairs) should behave in the organization. The researchers noted that faculty saw department chairs as part of the collegiate in-group, while department chairs saw themselves as partly administration and partly faculty (Souba et al., 2007). As a result, both the leader's and constituents' values were, for the most part, misaligned, and, over time, the constituents' expectations about effectiveness of the department chair's leadership style and approach declined. This particular study is relevant to this research because, although it was focused on another level of management, it did provide some insights about how some faculty may view effective leadership in higher education. Such implications are fundamental for understanding some of the overt and covert tensions between these two groups, especially if these issues evolve into conflicts, such as no confidence votes.

\section{Problems with perceptions about leadership in California's community}

colleges. The structure of leadership in community colleges, which is described as 
having a team or collaborative design, also appears to be at the center of how leadership is perceived (Shulock, 2002). Otara (2011), using Wielheim Wundh's study of perceptions between management and their employees, defined such perceptions as "the way we all interpret our experiences... [and such interpretations are used] to shape the climate and effectiveness of the working environment" (p. 21). Hargeaves and Fink (2008) asserted the current structure of leadership in higher education is no longer based on the lone-star leadership model but there is now a revival around the concept of collegiate leadership that includes a "network of people aligned through informal and formal...coalitions to lead" in their organizations (p. 229-331). Such coalitions are clearly evident in shared governance policies and laws that encourage management and employees to work closer together.

Hoy and Miskel (2008) suggested that such perceptions are common because leadership is something that is socially constructed. They defined socially constructed leadership as the "social process[es] in which a member or members of a group or organization influence the interpretation of internal and external events... organization of work activities, abilities, power relations, and shared orientation" within their individual context (Hoy \& Miskel, 2008, p.420-421). Sinclair (1998) also posited that the result of leadership being a socially constructed concept with different individuals having different expectations about leader may be a result of these individuals is also the result of individuals internalizing certain 
mindsets about leadership which are often muddled with the "legacy... of the archetype leader" with many assumptions about what such leaders look like (p. 177). In her study with both women and men executives, she found that gender and sexuality mattered because such leaders may lead or are expected to lead differently (Sinclair, 1998). For instance, she found the institutions with women at the helm may find themselves resisting her style of leadership because it sounds and looks different to them. While institutions with male leaders experienced less resistance to their leadership, at least at first, primarily as a result of the "dominant literature... [that is mainly] masculine" that can inform what leadership should look like (Sinclair, 1998, p.178). Similarly, Pratch and Jacobowitz (1996) studied the perceptions about what is effective leadership among 40 male and female graduate students in a leadership program. These researchers found that most male leaders were likely to demonstrate tendencies towards being agentic leaders, which they described as "being independent, masterful, assertive and...competent" (p. 203). On the other hand, female leaders were more likely to demonstrate communal leadership tendencies, which they characterized as having "needs for affiliation, lack of self-centeredness, [and] concern with others" (p.203). As a matter of fact, these researchers found that women were expected to demonstrate such communal tendencies and may have experienced negative perceptions and resistance when they used agentic tendencies. Although these researchers were unable to measure if men who adopted communal tendencies also experienced negative perceptions, they 
found that individuals in the environment were prone to evaluate male and female leaders differently using similar frames (Pratch \& Jacobowitz, 1996).

Overall, the literature is clear about how such mindsets and perception can influence the ways we think about leadership altogether. Therefore, if and when a group of individuals witness any type of disconnects and ambiguities about what effective leadership is in their institutions, there could be serious implications for these leaders. This may be especially true in education where the concepts of "shared leadership" has become prevalent among educators who believe they have a shared responsibility for educating their community through "shared visions... shared coalitions... [and] collegiate-leadership teams" and expect leaders to demonstrate a certain type of practice around these concepts (Lambert, 2002, 37 38). Perhaps, these votes are the effect of leaders who fail to manage such perceptions within these structures of work, particularly when such perceptions are that the leader is ineffective in his or her role and responsibilities within the context of these institutions (Otara, 2011).

\section{Problems with leadership expectations in California's community}

colleges. The literature described expectations about how leaders will perform their work and how responsive they should be to their organizations to be effective. Eckel, Hill, Green and Mallon (1999) described the salient role of the leader as a major change agent, who is developing significant and intentional processes of 
identifying critical issues within education, and effectively applying the change process to it (p. vi). Such expectations are not unfounded, particularly as leaders across the United States have grappled with finding and applying effective, largescale, school reform strategies in response to government-issued manifestos and policies for which they are accountable, and they have continued to struggle with the need for society and the economy to stay competitive among other countries around the world (McKinsey, 2007). The literature has also admonished leaders to find effective ways to work within their organizations and interact with institutional stakeholders, and to not be distracted by management fads and gimmicks for leading change on the campus, which can eventually create more problems within systems over time (Birnbaum, 2000).

In their study on understanding the limited roles of mid-level leaders at elite institutions, Westphal and Khanna (2003) asserted that there may be some dissonance between what constituents within an organization, especially faculty, expected these leaders to do, and the social control they have over leaders. This control can then impact the leader's decision-making abilities and, eventually, affect key stakeholders and constituents, especially students. These writers provided an example of how certain norms, rules, beliefs and values, such as social distancing, can indeed impact leaders and eventually control their behavior in pushing them to conform to certain behaviors. Leaders who refused to conform were usually "squeezed out" of positions or "ignored" (p. 367). If these findings 
are consistent for leaders on a larger scale, then how exactly are leaders with different leadership styles and approaches expected to survive such actions, without fear of being pushed out?

In another empirical study examining the effect of institutional forces in a working unit and how it can shape and influence its members about leadership, Gupta, Dirsmith, Fogarty (1994) investigated rules and structures in the workplace. These researchers interviewed 96 audit leadership teams from the United States General Accounting Forces to find out how the coordination and controls in organizations are used to control the task environment (their work) using internal and external forces. The results of the questionnaire showed that auditors often felt restrained by the external bureaucratic process prescribed for them, and saw good leadership as protecting them from outside forces and supporting them to get their work done. Meaning, these researchers found that, as the workers conformed to this bureaucracy, they experienced higher work performance. However, beyond the bureaucratic processes required by external agencies, these auditors performance also improved when their work fit their perceptions of meaningful work as defined by their internalized and social group structures about the work or their "idiosyncratic personal and group modes" for operating, as well as the task itself that was based on their expertise (p. 277). Thus, these auditors expected their supervisors to both support them in their roles, as well as focus on reducing external forces that can hinder their work. Additionally, these researchers found that 
supervisors who deviated from the norm and incorporated more "robust" ways of leading were viewed as more effective among auditors who were already highly experienced and trained to do their jobs (p.279). Gupta et al (1994) asserted that such environments can serve to squeeze out individuals who fail to conform to their pressures and norms within these environments. Although this study does not correspond to the salient issues being examined in the academy, it did offer some insights about additional expectations in highly complex systems that leaders should be cognizant of, because they are embedded in the culture and structure of the organization (Schein, 1993).

Leaders managing these perceptions. The question of do leaders know or are they aware of such perceptions and expectations about their leadership naturally comes to mind. M. Kuykendall in an interview with Schmidt (2009) and Smith (2003) found that institutional stakeholders who supported the vote most likely expected leaders to perform their roles a certain way, to be open and responsive to their concerns, as well as to maintain the values and culture within the organization. According to the American Council on Education (2007), such expectations about leadership and how leaders will perform are further validated through the responses of over 3,000 presidents from all sectors within higher education. The ACE (2007) report indicated that $77 \%$ of the presidents surveyed believed that certain behaviors or actions could pose risks to their leadership or cause a leadership crisis, such as 
failure to effectively fundraise and to secure private funds, especially during austere economic times. Other presidents $(38 \%)$ believed that technological advancement was a key problem area that they needed to have the skills to address, while others (20\%) believed that they had to build competencies to deal with "political polarization or erosion of public confidence" a trend they believed could have a "a major effect on the presidency" (p. 44). Another 4\% of these presidents indicated that "news cycles" and reports in any "media" were also newer trends affecting their presidency, for which they had to build strategies to address (p. 44). In this report, leaders appeared to be attuned to the types of behaviors or problems that could impact their leadership or shape perceptions about their overall roles. These respondents were able to clearly identify certain situations that could affect them professionally.

Thus, the extant literature indicated that institutional players may have different expectations and perceptions about leaders and leadership within their institutions. If leaders are unaware or fail to manage such expectations and perceptions, such as adhering to the internal and external demands in the environment and the norms and culture within the organization, there may be serious consequences for these leaders. As a result, the literature encourages leaders to be espoused to different styles and ways of practicing their leadership, ensuring that they are paying attention to how they are being perceived as they move through different cycles of work within the institution (Pratch \& Jacobowitz, 
1996). The literature has also shown that leaders are expected to adhere not only to the external forces and controls in the environment, but to conform to the norms and culture within the organization. In other words, leaders need to be cognizant of the specific contexts in which they work and how it can affect their overall leadership effectiveness.

Leadership identity. Beyond the leadership development theories that have conceived the many ways in which individuals become leaders overtime (Bennis, 2004; Dreyfus, 2004), there has also been literature on the importance of identities of leaders overtime and how identity can influence effectiveness. This literature is generally focused on two specific areas: how leaders see themselves and their leadership, and how others see or define good leadership. Curry (2000) suggested that the ways leaders think about themselves, also known as their leadership "personas", strongly reflects the many aspects of the things that can shape a person's identity and how he or she will act as a leader (Curry, 2000, p.22). Still, the literature about leadership and competencies has also delved into gendered approaches to leadership, so much so, that recently this literature has depicted feminine ways of leading as having an advantage in organizations over their male counterparts (Eagly \& Carli, 2003). 
Leadership personas. A cross-section of literature on leadership personas is focused on leadership position and power, and how these two elements can influence leadership and leadership identity. For instance, Eddy and VanDerLinden (2006) investigated the ways leaders identified themselves and are cognizant of the institutional elements that can increase their power and influence in the organization, as well limit their capacities to lead (Eddy \& VanDerLinden, 2006). In their study, a survey instrument was used to collect data from 910 college administrators, including faculty leaders, administrators and mid-level managers, and then applied to a content analysis approach to code emergent themes. Eddy \& VanDerLinden (2006) explored how leaders self-reported their roles within the context of their organizations into eleven (11) categories, to analyze the participants' responses. The eleven categories included having power, influence, knowledge, expertise, and being a change agent, to name a few. One of the key findings of the study was that there were multiple opposing views between constituency groups about what leadership is and how it is defined within the context of the organization. The results indicated that $47 \%$ of the respondents saw themselves as leaders because of the symbolic title of their positions, while others used "expanded definitions" of leadership, such as expertise in the classroom or advocating for students to position themselves as leaders, which were statistically significant (Eddy \& VanDerLinden, 2006, pp.15). These researchers also indicated that there were few differences between the ways male and female respondents 
described their style of leadership. However, these researchers found a significant correlation between the institutional structures that may have acted to constrain or accentuate the advancement of women, for example, which was evident in language and interactions between these groups. Another major finding was that members of academic affairs saw themselves as having more power and ability to enact change within the organization, more so than presidents and provosts. Other groups, such as those in student affairs, felt more empowered to enact change for the people they served (students), not necessarily over other administrators or other personnel groups, while presidents and provost felt the least empowered (Eddy \& VanDerLinden, 2006). These findings are important because it demonstrates the types of mindsets about leadership and power that be propagated within groups and across the institutions. This study also highlights the need for leaders to be aware of themselves as leaders, as well as the structures and mindsets among constituency groups that can ultimately impede or support their roles in the presidency.

Leadership and gender identity. The literature on leadership identity is equally focused on gendered ways of leading and on the dynamics between androcentric or masculine and feminine traits, beliefs, attitudes, values and practices in leadership. The term gender is defined as the "role, not biological sex" and refers to "women and men as a social group" (APA, 2006, pp. 71-73). This type of leadership literature has also shifted from the great-hero ideologies usually applied 
to men or male-centered leadership (Bass, 1990; Burns, 1978). Some writers are more inherently predisposed to provide the optimal approach to leadership, which they see as transformational (Booysen \& Nkomo; 2006). Still, other authors, using discourse analysis to examine this literature and these shifts in the literature that give women the advantage over their male counterparts, asserted that such shifts are both temporary, cyclical and they can follow whatever movements and reforms are current within society (Amey \& Twombly, 1993). Others concur with this analysis, suggesting that such cycles of acceptance for feminine leadership are better aligned with certain social movements, such as the feminist movement, the civil rights era, and affirmative action, which gave women the advantage to occupy spaces normally dominated by men (Eddy, 2008).

For example, Tedrow and Rhoads (1999) observed the relational (feminine) or participatory and collaborative ways of leading, contrasting it to instrumental (masculine) practices, further polarizing the differences between men and leaders. These writers also defined instrumental or "instrumentalism" ways of "knowing" as being rational in taking strategic action, which are closely "associated with being masculine" (pp. 1-2). Moreover, these writers described relational ways of leading as being akin to a feminine approach, an approach associated with leadership focusing on being participatory, "building coalitions, [and] sharing power" (pp.2-3). They also considered the perceptions of leaders about themselves, their leadership, as well as the perceptions of the people they lead, and, more often than not, they 
have found that these leaders felt that there were tensions between what people idealized as the optimal way of leading, and whom they consider an effective leader, regardless of how the individual actually performs as a leader (Tedrow \& Rhoads, 1999). Essentially, these writers purported that such dyadic connections between gender and leadership also may be used to influence how being masculine or feminine matters in leading organizations (Tedrow \& Rhoads, 1999).

As a result, these aforementioned leadership and gender frameworks are believed to have proliferated society's understanding about leadership overtime, by promoting and challenging the differences between men and women, and by emphasizing the social constructs of gendered differences and a one-size-fits-all mentality about the leadership or management approaches of organizations (Risman, 2004). According to Eagly (2007), some leadership literature still viewed women as being "lauded [with] having the right combination of skills and leadership" to lead organizations in the $21^{\text {st }}$ century (p.1). The American Council on Education (ACE, 2007) also spoke about this shift in education. ACE (2007) found that as of 2006, the hiring percentages of women were "higher for presidents in the public community colleges systems (28\%)," than in previous times in history (p. 51). Eagly (2007) asserted that, although organizations claim they prefer leaders who emphasize collaboration, participation, and transformation, there is some disconnect between what they are emphasizing and what is actually happening. Although this study asserts that this progress is still too slow, it also recognizes that 
both women and men may ascribe to both masculine and feminine ways of leading, which may or may not include being highly collaborative or responsive to constituents in the academy.

To further support these assertions in the literature, Eagly (2007) examined these concept in her empirical studies about the advantages and disadvantages of gendered leadership in the academy. The results indicated that: (a) women in male-dominated positions face obstacles that their male counterparts do not face, and (b) women still face stereotypical and "prejudicial" attitudes that include "preferences for male bosses over female bosses" also evidenced in the 2008 Gallup polls during the recent presidential campaigns (p. 7). In addition, this researcher found that women were still expected to demonstrate that they can adopt behaviors associated with masculine traits, such as "toughness and competitiveness" to gain credibility in male-dominated roles (p. 6). Furthermore, Eagly (2007) also identified the ways in which constituents may expect their leaders to behave, as well as the types of assumptions that leaders may bring to the position in an effort to secure their legitimacy as the leader. However, unlike their male-counterparts, these perceptions and expectations about women and their leadership in the organization may be flawed and may continue to persist in this environment if such perceptions are influenced by prior taken-for-granted assumptions about how leaders will perform and act in these positions. 
For this study, a major criticism about these types of leadership literature is that it not only still privileges masculine leadership, but it has influenced the perceptions and expectations about how women will perform and be different, perhaps better, when compared to men in these leadership roles (Butler, 1990). Such leadership literature can further create stereotypes and cause stereotype threats for all types of leaders (Steele \& Aronson, 1995). The term stereotype threat refers to "being at risk of conforming, as self-characteristic, a negative stereotype about one's group" (Steele \& Aronson, 1995, p.1). As a result, leaders may face additional pressures to perform in ways in which their constituents expect them to behave, even to the point of adopting gendered types of leadership (Butler, 1990), which can further result in challenges associated with gender identity, stereotypes, and threats (Acker, 1990; Eagly 2007; Eagly \& Carli, 2003; Hayes, 2010; Switzer, 2006). In addition, this study also questioned the validity of the literature that gives women the advantage in leadership over their male counterparts, and suggests that research should move away from these paradigms and focus more on detailing the ways in which leaders actually practice leadership effectively in the academy (Acker, 2010). Other researchers agree with this viewpoint, emphasizing that the type of leadership needed in education should no longer be centered on who is leading or what styles they use, but should instead be focused on the structure of the organization and how leaders practice leadership within these contexts (Spillane et al., 2004). Spillane et al. (2004) have suggested that leaders, even those who 
incorporate a collaborative approach to leadership in higher education, should espouse a leadership practice that readily acknowledges the organization's contextual factors (i.e., school's functions), situational dynamics (i.e., structural norms, values, and beliefs of the organization), and artifacts (i.e., the symbols and traditions that have a meaning to the organization), all of which can influence leadership practice in both positive and negative ways (pp. 2-11). Repeatedly, the literature has clearly delineated the need for studies to examine the dyads that may exist in leadership and how such relationship can result into a crisis event for leaders and their institutions. Thus far, this study has discussed how leadership and theories about leadership; competencies; styles, stereotypes, perceptions, and expectations can color the ways leaders think about themselves and how others may think about them as leaders. Concerning no confidence votes, the primary position of this paper agrees with the literature that is focused on evaluating leadership on the premise of how leaders practice within their institutional contexts and are able to reasonably meet the needs of the community in which they serve. In many of these reports institutional players singled out the leadership's incapacity to lead because of the type of style and behaviors they perceived as inappropriate, and in opposed the leadership and their agendas, the sponsors of the vote used a lack of confidence in the leadership as an indictment against the leader and inducement for these votes. Contrastingly, the counter arguments of these leaders about such evaluations of their leadership is that they were doing what they were hired to do 
and that the votes were evidence of the "inability of all players to unite around a common agenda for the institution" to move the organization forward (MacTaggart, 2012). Additionally, boards are then left to evaluate this conundrum between these two entities, and they may or may not find any premise for removing these leaders on the grounds stated by these votes, particularly when these boards may have initially approved the agendas and actions of these leaders. For this reason, boards may also decide to simply deny the demands of the faculty, if they find such demands as unfounded and decide to support the leader, which institutional players may regard as a biased response to their concerns (Schmidt, 2009: Tierney, 2007). Therefore, understanding the contextual factors in which leaders practice is another crucial area that will be examined, to further increase knowledge about how this context can lead to these resolutions against the leadership.

\section{Theme Two: Contextual Factors Contributing To the Vote}

Another reoccurring theme in these NCV reports is the concerns about the contextual factors that emerged during a breakdown in the interrelationships between these leaders and institutional players while working closely together, such as during collective-bargaining negotiations or strategic planning initiatives, for example, (Schmidt, 2009; Smith, 2003). These failed partnerships were also blamed on the behaviors of a flawed leader, who may have been perceived as being incapable of communicating effectively during such routine cycles of work, or 
dismissive of shared governance tenets during specific-context related situations, as detailed in Chapter One (Smith, 2003). As a result, the following sections will focus on certain context-specific factors that may have led to the vote; identify the possible antecedent conditions that may be attributable to these votes; as well as examine additional theories for explaining these patterns, and offer possible implications of the vote on both these leaders and their organizations.

For the most part, leaders understand that their interactions and relationships within the context of their institutions are crucial interrelationships that can impact their effectiveness as leaders. The American Council on Education (2007) validated this assertion through the responses of over 3,000 presidents from all sectors within higher education. The ACE (2007) report indicated that $77 \%$ of the presidents surveyed believed that certain behaviors or actions could pose risks to their leadership or cause a leadership crisis, such as failure to effectively fundraise and to secure private funds, especially during austere economic times. Other presidents (38\%) believed that technological advancement was a key problem area that they needed to have the skills to address, while others (20\%) believed that they had to build competencies to deal with "political polarization or erosion of public confidence" a trend they believed could have a " a major effect on the presidency" (p. 44). Another 4\% of these presidents indicated that "news cycles" and reports in any "media" were also newer trends affecting their presidency, for which they had to build strategies to address (p. 44). Whatever the specific-contextual factor, these 
leaders understood that their institutions would make them accountable for any and all situations that arose on during their leadership and how it could impact them. Therefore, the leadership's behaviors during shared governance cycles of work and their overall practice of communication will be examined.

Smith (2003) asserted that some reoccurring contextual activities and factors within the organization led to the votes in the cases she reviewed, such as:

1) Forty percent of the votes emerged out of disagreements and frustrations during collective bargaining cycles and were mostly given by faculty unions against leaders they thought were "unfair" during these processes.

2) A third of the votes were based on a lack of faculty participation in organizational changes and decision-making processes, were faculty may have felt excluded from certain process.

3) Other contextual factors involved technological updates, unfair terminations and fiduciary concerns and appropriations that leaders were responsible for (p. 3-4).

More specifically, Smith (2003) inferred that, in the California system, some of the narratives of the faculty suggested that these votes were enacted on leaders who violated the shared governance process, a process through which leaders and constituents are required to consult with each other, and share responsibilities over specific decision-making areas (AAUP, 2005). The organizational and management literature have shown that the structure of any organization, which is described as 
the ways in which roles and functions are assigned and divided, can influence the formal and informal culture within that organization, and vice-versa (Schein, 1996; Schermerhorn et al., 2012). Culture refers to "shared actions, values, and beliefs that develop within an organization and guides the behaviors of its members" (Schermerhorn et al., 2012, p.6). Thus, in living systems like community colleges that may have several complex structures coexisting together, there may also be different sub-cultures within departments, divisions and across all types of divisions of labor, which leaders must help to develop, maintain, and be responsive to their needs (Schein, 1996). As a result these indictments against the leadership and the antecedent conditions surrounding the culture of interactions between leaders and constituents during high-stake activities, particularly as it relates to shared governance structures and processes, are fundamental to understanding the possible factors that can lead to these no confidence votes.

Structure of shared governance and its impact. Shared governance within the California Community College system is a highly formalized structure, also known as the California Assembly Bill or AB 1725 enacted in 1988 (Senate Bill, 2005). AB 1725 defined the community college's role and mission in educating California's students and their communities in the state, as well as it defined the structure of control and governance, such as "shifting the power from the legislature back to local boards" and districts (Livingston, 1998, p. 5-6). Therefore, local boards were now accountable to local communities as well as California's 
Community College State Board and Chancellor's Office, they were now more responsible for budgeting decisions, meeting certain goals such as maintain fulltime to part-time faculty hiring ratios, offering remediation and vocational programs to community agencies and all students, etc (Livingston, 1998).

Scroggins (1997) asserted that the AB 1725 law also defined the roles that faculty and faculty senates should play in the decision-making processes or shared governance with administrators concerning academic and personnel matters, as well as other literature suggested that staff and students in the community colleges were afforded shared processes by this law as well (ASCCC, 2002; see Education Code 70902 (b), Section 53200-53204). More specifically, this formal agreement is considered a means for increasing a collegiate culture around decision-making, which stipulates the eleven specific areas of decision-making that both faculty and administrators must participate in together (Education Code, Section 53200; Community College League of California, 2012; Gabriner, 1995). Therefore, tighter participation between administrators and faculty during certain activities like collective bargaining, hiring other faculty, evaluation of their peers, etc would become the new norm.

The meaning and implementation of this shared governance model is still highly challenged in California's community college system, which one faculty blog described as increasing tensions among constituents, like "getting an unsigned blank check that you can never cash, or like having a recipe and all the ingredients, 
but no instructions for putting it together" (anonymous blog, 2008, para. 2). What started out as grass-roots movement to support the roles of the faculty of community colleges (CC), who mostly migrated from out of the $\mathrm{K}-12$ setting, became the policy that allowed the faculty in this system to have similar structures like their counterparts in universities, and to maintain funding by increasing ties to local communities (Livingston 1988). As a result, AB 1725 was now the gold standard for how governance should work in this system, even for institutions that may have been functioning successfully or already using similar frameworks. Essentially, this law left the implementation of this statute up to the colleges, and without a formal model for practice, there has been contention over just how much input faculty and other groups will have in certain matters (Academic Senate of California Community Colleges, 2015). Livingston (1998) agreed that the words shared governance and its meaning have been a place of contention for institutional players, but maintained that even though the actual words shared governance does not appear in the actual law; the term became the accepted frame to describe the law overtime. For others the disagreement over shared governance begins and ends with the actual terms used in the law. More specifically, there are disagreement over the certain terms within the law that requires leaders to consult with, "rely primarily" on the judgment and decision making of faculty or administrators, as well as areas were leaders will ensure "participation" or "mutual agreement" of faculty and other institutional groups in academic and professional matters, also known as the $10+1$ 
areas (Academic Senate for California Community Colleges, n.d., p. 19-20). Table

5 below illustrates these areas of reform and governance structure in this system.

Table 5

Shared Governance Law-AB 1725 for California Community Colleges

\section{Summary Areas of Assembly Bill (AB 1725) California Community}

College Reform

(Shared Governance)

1. Curriculum, including establishing prerequisite requirements

2. Degree and certificate requirements

3. Grading policy

4. Educational program development

5. Processes for program review
6. Standards or policies regarding student preparation and success

7. College governance structures, as related to faculty roles

8. Faculty roles and involvement in accreditation processes

9. Policies for professional development activities

10. Processes for institutional planning and budget development

\section{Other academic and professional matters as mutually agreed upon.}

Note. Adapted from 4faculty.org website, retrieved on December 21, 2015

Similarly, Smith (2003) suggested that there were still some level of confusion over an acceptable shared governance model for the individual campuses, particularly with the eleventh are that requires consultation and mutual agreement on academic and professional areas. Without a clear model or definition of these 
areas, faculty and other groups have continued to challenge any action or behaviors suspected of violating these tenets, and some groups may have to resorted to using no confidence votes to demonstrate their growing disaffection against administrators (Smith, 2003). In addition, both faculty and administrators have voiced different sentiments about the overall shared governance law, and they have voiced either support or resistance about how it has been implemented. For example, Shulock (2002) advised that proponents for this type of governance see it as a means of "flattening the organization" and fostering "good relationships" (p. 4), while opponents see it as a hostile takeover or a "handing over of the institution to the faculty" (p. 4-5). Bahls (2014) suggested that while faculty viewed shared governance as a way to ensure that leaders will be transparent and include them in the decision making process for overseeing the organization, leaders and boards may not feel the same. As a matter of fact, Bahls (2014) suggested that leaders often saw the law as an "obligation to consult with faculty" rather than a collaborative process, partly because they do not perceive that faculty members understand the work of leaders and that they do not have the training or insights to make certain decisions (para. 12). As a result, such leaders my resist this policy at times and may deliberately sidestep certain activities to get their jobs done.

Another criticism of shared governance in California's community college system is that the implementation and even the design of this policy may be flawed and misleading, because such governance structures are difficult to attain at every 
level, especially if all who govern do not shared the same level of power, responsibility, or accountability. Meaning, both leaders and faculty should experience the same level of accountability for decisions being made within the institutions. Without an equally shared accountability system in place, institutional players will always be fixated on selecting a human agent or leader to bear the sins, or be the scapegoat for their institutions (Gamson \& Scotch, 1964).

Smith (2003) advised that not all community colleges have experienced this tension over shared governance. She asserted that some institutions within this system have successfully implemented a shared governance model, before and after the law actually existed. Smith (2003) speculated that the institutional culture and context were key in such instances, and institutional cultures that were already highly collaborative were more likely to make the transition more smoothly. Olson (2009) concurred with Smith's (2003) hypothesis about the structure and culture of community colleges being suspect, suggesting that sometimes social and internal belief systems of the faculty and leaders in this system can contribute to such tensions. He suggested that the faculty, who see themselves as the university may still be using an adversarial paradigm, such as "You're the administration, and we're the university," further adding to the increased tensions in these participatory models (p.1). Olson (2009) and Collins (2002) observed that there are many obstacles to shared governance which are usually coupled with strained 
relationships between these key agents, alongside other challenges, such as a lack of fiscal and human resources available to fully realize this reform.

In sum, these writers have asserted that both the context and culture of the institution were significant factors in how these regulations were understood and factors in how leaders were perceived in light of this law. Birnbaum (1992) in a later examination of the roles that leaders and constituents played in the academy found that the interactions between constituents can become even more strained during high-stakes activities that require them to work together in a highly structured or formal context, such as accreditation or budget allocations. A major implication of these votes on leaders need to be aware of these cultural and political elements that may be present in their organizations, even before they oversee these institutions and that such knowledge may help leaders manage perceptions and expectations for responding to this law. As a result, leaders who fail to be commit and be responsive to this law may find that they will quickly "lose creditability with the faculty if they short-cut agreed-upon processes (Bahls, 2014, para.11).

Antecedent conditions in shared governance structures. Smith's (2003) work supported Birnbaum's (1992) findings, and suggested that some high-stakes will require leaders and faculty to work together, and may be central to some of these votes. Thus, such interactions and past interactions may have been antecedent 
conditions already present in the organization and shaping the outcome of any new highs stakes activity between leaders and their constituency groups.

a) Perceptions about contextual factors. A major contextual and antecedent factor that can influence the way shared governance can impact the way work is arranged in these institutions. As previously noted, the responsibilities of the administration and the faculty are structured according to their specific roles and tasks within the organization; however, shared governance laws can create overlaps in the functional areas and increase tensions between these groups. For example, (Birnbaum, 1998) advised that faculty are hired to teach and do research and service, whereas presidents are hired to fundraise and garner support from stakeholders. As previously noted, Birnbaum compared these arrangements to Weick's (1976) loosely coupled or tightly coupled systems, a system used for balancing and coordinating control, power and decision making in the organization. Weick (1976) described loose coupling as the major component in large, complex systems and structures that operate within both formal divisions of labor-based expertise and functional areas, such as faculty divisions, the administration, etc. He also advised that these coupling structures are generally stable and standard in educational organizations, similar to U.S. political systems and structures; however, they are not completely static and can become more loosely or tightly linked around threats or opportunities, such as policies or funding, for example Weick( 1976). 
Weick (2001) would later advise that these types of structures have both advantages and disadvantages, which are based on the power distances between groups and can be less formal and have hidden connections and ties. In fact, he later added that such systems "rely on trust and presumptions" in their invisible design (p. 401). In addition, he advised that these coupling can be further constrained or supported by the frequency of the interactions between these groups and whether or not they voluntarily participated in certain partnerships or were obligated to do so Weick (1976). Therefore, collective-bargaining negotiations can become an area where leaders and faculty unions must interact and communicate with each other more frequency and over longer periods of time. As a result, such prolonged interactions not only minimizes the distance between these groups but they can become highly frustrating periods of interactions between both groups than can further shape or change the perceptions about the leader.

By applying shared governance precepts, which are rules for how individuals will interact, to these loosely or tightly coupled environments it may be possible to see how the perceptions about leaders could change overtime. Weick (1993) suggested that expectations about the capacity of leaders and what they can actually get done may be a part of the problem. Weick (2001) further admonishes leaders that institutional players may believe that "management has a [more of a] direct impact on design" than they really do (p. 68). He advised that managers, including presidents, have traditionally had less "discretion" to get things done and 
that they tended to use "formalized relationships [that can] make it easier or harder to get things done" (Weick, 1993, pp. 68-71). As a result, institutional players within this structure may feel the need to act on their assumptions and expectations about the leader's capacity to pay attention and even to design or redesign shared governance in their institutions. Other researchers have advised that a major challenge for most leaders in California's community colleges is to conceptualize and enact an ideal and realistic model of shared governance for their specific institutions (Gabriner, 2005; Shulock, 2002). Therefore, frustrations over the leaders violating shared governance precepts or their lack of commitment to the process can result into these boiling point votes. Such boiling points may accelerate even faster when institutional players have had to content with a series of leaders who have allegedly violated this law in the past, and when new leaders continue with such trend of devaluing these policies.

b) Institutional culture among groups. Other scholars suggested that such systems of controls and interactions in higher education may be more social, psychological and covert in nature (Eagly \& Carli, 2003; Neusman \& Benisom, 1990). As a result, institutional players may want to see strong commitments to shared governance and other cycles of work to build up their confidence in these leaders (Bahls, 2014). These institutional cultures may evaluate the leader's commitment to shared governance by what they communicate, the opportunities they create for faculty to participate in decision making processes, and how open 
and transparent they are about the state of the institution (Bahls, 2014; Gabriner, 1995; Smith, 2003). For example, Caton (2007) agreed with this premise and suggested that sometimes institutional players decided whether or not they will support, influence, or impede how leaders function in the organization depending on how well they complement their current systems. Using women's leadership as a premise, Caton (2007) surveyed president respondents across the United States, and found that, although most leaders did not ascribe to notions of gender being a problem, gender negatively impacted their "ability... credibility, old boys" networks, networking opportunities, governing board, politicians, demeanor and isolation" within the organization (p. 12). More specifically, respondents either agreed or strongly agreed that their leadership perceptions, as well as the perceptions of what others thought of them, were strongly influenced by personal factors, such as gender and other aforementioned factors. Furthermore, this researcher asserted that, regardless of the leadership approaches of these presidents, it is possible that certain leaders may struggle to "achieve respect and confidence" in their organizations (p. 13). Thus, in some organizational contexts, women leaders, for example, may still have to do more planning to explicate their ways of leading to garner support from members, especially in institutions that never experienced a woman president. In general, the inference here is that some leaders may need to take the time to explain their brand of leadership and practice to their 
institutions to manage certain expectations and perceptions about their leadership and commitments to shared governance, for example.

c) Protecting certain values and beliefs. Other institutional scholars have suggested that community colleges are complex systems that protect the symbol of who they are and what they are commissioned to do, although "they [may be] failing to meet their mission evidenced by their drop out, stop out, and low graduation, job placement, and transfer rates overall" (Brint \& Karabel, 1989, p. 337). These critics and others have made similar assumptions about other sectors of higher education that are designed to protect itself (DiMaggio \& Powell, 1983; Ramirez \& Bradley, 1990), and how these organizations have survived because they have learned to protect their technical core, such as the teaching and learning within the organization, from public scrutiny (Elmore, 2004). One way that organizations do this is through isomorphism, a three-pronged process wherein members first mimic other well-established organizations within their sector, field or environment, to ensure survival in that environment, then create policies to buffer or protect itself from outside agencies and scrutiny, and then develop formal and informal artifacts to influence the ways in which actors will perform their work and to influence culture (DiMaggio \& Powell, 1983).

Several scholars have also written about the ways in which higher education, for example, has been designed to keep American society believing in the achievement ideology that, if they work hard, they will succeed (Bowles \& 
Gintis, 1976; Apple, 2006), and failure to do so is usually the leader's fault. These scholars see this as an embedded way of thinking within the institution, which acts as a buffer against anyone who might question its legitimacy and a means for survival (Meyers \& Rowan, 2006). Other writers suggested that this type of buffering exists inside the academy, especially evident in the ways technical areas of the academy, which are often considered off-limits because they are embedded in the work of the faculty with academic freedom, making it difficult to evaluate or to correlate with the failings of teachers, not just students (Elmore, 2004). Elmore (2004) asserted that such systems or individuals act as safeguards and may be responsible for creating meanings about situations, and protect their organizations from major changes and disruptions, especially from external scrutiny. For instance, such buffering actions may be applied to community colleges, making it difficult to really know when a president is truly succeeding or performing as expected, unless other symbols or signs are involved, such as the president bringing in millions of dollars into the organization, or there is an outright scandal in the leadership (Shen \& Cho, 2005). As a result, these organizations can remain minimally disturbed by critical events such as votes against the leaders, and at the same time they are designed to protect leaders and constituents who are performing well or not in them.

d) Leader's role in such shared governance systems and cultures. Leaders who understood the history, structure, and culture and antecedent conditions within 
these organizations, may still decide to take on the presidency anyway, even at the risk to their leadership. For example, in the no confidence vote case of Dr. Jerry Sue Thornton at Cuyahoga Community College, "recognized nationally for developing [student success and workforce development] innovative programs," the faculty rationale for the vote was that "she isn't fit to lead the 22,000 student college" (Farkas , 2010). This writer noted that the counter-narratives from other constituents within the organization suggested that the environment was hostile and the real reason for the vote was a conflict over "who would control the college...the president or the faculty union" (Farkas, 2010). In this scenario, Thornton did not provide any comments or reaction to the vote, but she did not appear surprised considering the environment she had grown accustomed to leading for her six years as the president. As a matter of fact, this leader suggested that she "was not surprised" to get the vote after union contracts were approved, and she believed that the antecedent conditions of a difficult faculty contract negotiations and fears that more adjunct faculty would continue to outnumber the tenured, were part of the rationale for the growing disaffection regarding her tenure (Farkas, 2010).

Others scholars suggested that leadership style and practice matters, particularly when leaders are not perceived as being supportive of certain models of collaboration, regardless of mandates. For example, Shaw (2005) asserted that constituents within higher education may have "deepened antagonisms within the academy," supporting the claim with his analysis of the assumptions that faculty 
may perceive administrators as using "corporatization" to manage the academy, which can breed malcontents among the faculty and lower morale in the organization (p. B.13). Thornton (2004) also supported these claims via research on corrosive leadership, suggesting that these tensions may have been increasing since the academy began shifting away from the faculty center model of leading, to a more corporatist model overseen by managers, which "centers around economic development and capitalism" (p. 162). Trombley (1997) asserted that the shared governance movement in California's community colleges (CCC) goes against such types of management style, especially as it was established to renew the collegiate model of leadership in community colleges.

Altogether, the literature review provided insights about several contextual factors and antecedent conditions that can give rise to these votes against the leadership. These factors may be associated with the behaviors or actions of the leader during certain activities, such as during shared governance routines that can then incite a no confidence vote. In addition, it may be influenced by the culture, values, belief and certain nuances within the context of the institution that leaders may not be aware of or may violate. Thus, this literature presented a quandary of sorts because it is especially difficult to pinpoint a trend or make connections about specific factors that can cause these votes, unless one study's such patterns and relationships between leaders and their institutions over time. Thus, this gap in the 
literature requires additional and in-depth research to understand the specific contexts and realities of these votes in different organizations.

\section{Theme Three: Communication}

Smith (2003) noted that a lack of communication was at the heart of many of these NCVs against the leadership. She highlighted that even with strong partnerships and committees already in place between leaders and institutional players, these votes were still unavoidable when certain lines of communication were broken or failed. The number of votes that were based on a lack of communication or consultation with faculty, such as regulated by $\mathrm{AB} 1725$, were not provided in her study, but it does appear that this attributable factor was a major part of the rationale for the vote. Smith (2003) also asserted that retrospectively, many leaders agreed that there were bottlenecks in these formal structures for communication and that, perhaps, "more proactive communication" prior to decision-making would have prompted different results and steered of a no confidence vote (p. 8). Overall, communication failures appeared to be a core central theme in these votes (Tierney, 2007).

In higher education institutions, including community colleges, the culture of communication can be described as embedded lines of communication, which are drawn along the divisions of labor in the organization (Schein, 1996). Such communication usually refers to the ways in which communication is transmitted, 
received, interpreted, and cycled between the sender and receiver, who, in this case, are the administrators and faculty in the organization (Schermerhorn, et al., 2012). Management literature describes communication as the "glue that holds collaboration and coordination together," as well as the "life blood" of the organization, because "all behaviors, good or bad" in the organizations must flow through this source (Schermerhorn et al., 2012, pp. 242-243). Other management literature suggested that, although there are many channels through which members in an organization can communicate with each other, such as face-to-face or email communication, leaders are burdened with the responsibility to carefully use channels to which members will be responsive (Gido \& Clements, 2014).

Perceptions about a lack of communication. Bahls (2014) and MacTaggart (2012) suggested that communication styles and frequency were crucial factors in how leaders were perceived in their institutions, and lack of communication could be as simple as an oversight of the part of the leader to as serious as institutional players feeling ignored. For instance, MacTaggart (2012) asserted that when leaders violated certain communication expectations or failed to communicate, it could lead to their demise that not even their boards cannot save them from. He identified poor leadership communication style as "arrogance...f failure to listen to [constituent's] concerns or take them seriously, habit of issuing top-down edicts... or [being] dismissive" as examples (para.11). Other criticisms about the leader's communication style included shouting or 
yelling at institutional players, using improper semantics, or lack of transparency when they did communicate (MacTaggart, 2012; Schmidt, 2009; Smith, 2003).

On the other hand, the responses of the leaders about such allegations on their communication style were either denial or acceptance. For example, Smith (2003) found that leaders believed that they were communicating often with constituents during committees and other forums, and that they used different strategies to maintain on-going communication in their organizations, such as newsletters, attending departmental meetings, etc. For the most part, these leaders agreed that communication lines can always be improved in their organizations, but they questioned these allegations that communication was poor, particularly when they tried to meet with constituents and were met with opposition, resistance and even threats (MacTaggart, 2012; Smith, 2003; Wright, 2007). Smith (2003) also found that institutional players also laid this allegation against the faculty when after consulting with leaders, the groups "did not receive what they wanted and decided to express their dissatisfaction through a no confidence vote" (P. 8). Bahls (2014) asserted that leaders who fail to practice open communication may be perceived as being disrespectful and will find it difficult to improve relations and build trust with constituents. Gido and Clements (2014) also suggested that leaders can avoid such communication pitfalls by being intentional in creating a communication plan that effectively conveys the accurate meanings or the intentions of their messages, which others suggest can further reduce or limit any 
interference (noise) or misinterpretations that members might have (Schermerhorn et al., 2012). Still, Smith (2003) advised that even with "ample communication and consultation" leaders may still experience a vote during times of extreme stress and ambiguity and skillful communication is an imperative. As a result, leaders may need to be aware of how different groups communicate or prefer to be communicated with, or they may run the risk of alienating or neglecting certain groups through strained lines of communication (Schein, 1993). Thus, depending on how and when information is communicated in the academy, constituents may determine that leaders did not communicate with them, or leaders may believe that faculty or staff did not communicate their concerns in a way in which they could both understand or find meaningful.

Communication problems during a no confidence vote. Several of these no confidence vote cases alluded to failed communication in the academy as the prime rationale for these votes. In addition to the aforementioned case of Harvard's Lawrence Summers, other high-profile cases also highlighted communication styles as the crucial problem. For example, Holly Moore at Shoreline Community College in Washington State also received the vote of no confidence from $77 \%$ of the 156 faculty, who voted in February, 2005 (Chan 2005; Perry 2005). Members of the faculty were concerned about her "top-down communication style and unfair treatment of the faculty" (Chan, 2005; Faculty Senate Minutes, 2005). However, a major antecedent condition that may have contributed to this event was the fact that 
this institution had already undergone a very public and embarrassing nepotism case involving their former president Gary Oertli, predecessor to Moore, and they were five years later admonishing their current leadership over her communication styles. The college's Board of Trustees at the time tried to give her an incentive to stay, a $\$ 25,000$ raise in salary, but she eventually left the institution a few months later, in August 2005 (Chan, 2005). Although there was no narrative from the leader about other reasons why she left, it is possible to infer that she may have left on account of this vote against her leadership. Other researchers have suggested that such strained lines of communication are typical in organizational life, including the academy (Birnbaum, 1988), and, although it could lead to conflicts between the sender and receiver, it can also present opportunities for groups or individuals to find more meaningful ways of communicating (Schermerhorn et al., 2012).

Communication theories. Jackson (2004), using Sherif and Sherif's (1964) Realistic Conflict theory, suggested that even with open lines of communication, the dynamics between in-groups and out-groups, or in this case faculty and administration relationships, can be so strained that continued contact and communication may fail to reduce the conflict between them. This was also evident in the relationship between Summers and the faculty he had already offended by his comments, and these faculty members were ready to give him a second vote prior to his abrupt resignation (Brush, 2006). As a result, boards who evaluate such 
concerns against the leadership, may have no other choice but to end the tenures of such leaders, if such concerns can be substantiated.

Other communication scholars have suggested that the simple exchange of information and interaction between groups can also lead to judgments about how individuals are expected to behave (Burgoon \& Dunbar, 1999). Regardless of the realities or situations in the social exchanges between individuals and groups, their expectations about how the other will behave can be violated and, as a result, lead to conflicts or other types of outcomes (Burgoon \& Dunbar, 1999). More specifically, leaders and constituents may both have expectations about the outcomes of their interactions with each other. Such expectations could include assumptions that leaders will consult with the faculty when making decisions in the organization, or that faculty will support the leader in their right to make decisions that are crucial for the management of the organization. Whenever such expectations are unmet, an expectation violation occurs (Burgoon \& Dunbar, 1999; Schmidt, 2009; Schermerhorn et al., 2012).

According to Burgoon \& Dunbar (1999), expectation violations, which can be both positive or negative, are another way for understanding the culture of communication in organizations, especially in regards to how such communication can impact individuals. More specifically, Burgoon, Stacks \& Woodall (1979) suggested that during conversations, especially face-to-face, "implicit messages", about how individuals will communicate can be violated, especially as these 
messages relate to gender and race (p.153). Similarly, individuals may also have low expectations of how others will perform; as a result, if leaders exceed these expectations, there is usually an immediate attraction to support and reward the leader with acceptance. Conversely, high expectations, especially those compounded by stereotypes about race or gender, can have negative implications on the leader as well (Acker, 2010). As a result, assumptions about time, distance or space, or gender behaviors during communication, for example, can be violated and can have either negative or positive implications for the individual communicating.

For instance, Switzer (2006) studied fifteen presidents in both two- and four-year colleges, found that some of these leaders felt like they violated expectations about how they would behave as a college president, especially after rising up from within the ranks of the faculty and stepping into their presidential roles. Switzer, using Weick's sensemaking approach, asked leaders to construct their journeys to the presidency. Most of these leaders provided insights about their struggles and triumphs for getting to the top, such as playing the scholarship and engagement games to secure their place as a member of the faculty and a team player among the administration. However, for some of the leaders, they recognized that once they got to the top, they tried to implement their own brand of authentic leadership, and, because it went against the expectations of the faculty, they were squeezed out of their positions (Switzer, 2006). In addition, one of these presidents suggested that she had to convince one board member to give her a 
chance at the presidency, especially after he declared that he would never consider a woman to lead that organization (Switzer, 2006). An inference that could be made from the study is that such expectations about the ways leaders will perform or practice is may be judged through the actions and language that leaders use to communicate. Thus, leaders may or may not realize that they will bear the burden of behaving in a certain ways and are bounded to certain ways of performing their work (Meyers \& Rowan, 2007), and they will need to use both formal and informal ways of communicating to be effective. Other authors have agreed with this prognosis, observing that failing to recognize these expectations and perceptions can have major implications for any leader (Birnbaum, 1988; March \&Weiner, 2003).

Thus far, the contextual factors presented in this literature review highlighted the factors that can either support or impede leaders in their work. These factors may emerge out of certain antecedent conditions that were already present in the institutions, such as shared governance laws, expectations about leadership performance and support of institutional values and cultures, etc. Similarly, leaders may find that they easily violate such expectations and trust in their relationships with constituents, when they fail to communicate openly and effectively, respect certain values or traditions within the institution or find creative ways to bridge the divide between themselves and other institutional players. As leaders manage and make improvements in these institutions, they may also need to 
take the time to explain their leadership decisions and behaviors as a way of managing certain perceptions and expectations about their leadership practice to be successful.

\section{Theme Four: Other Implications and Consequences of No Confidence Votes}

As previously highlighted in earlier and in Chapter One, the outcomes and implications of these votes are varied and sometimes unpredictable. Although the main consequence of a no confidence vote is that the leaders is "incapable of leading and must go" (MacTaggart, 2012). It may be fair to say that the consequence is either that the leader exits the institution, or retains their position with the support of their boards. However, from the review of the literature it also appears that such sanctions can have many far reaching consequences on these leaders, even beyond the initial evaluations and responses of the boards after these votes, and can affect leaders even when they have left these institutions. In addition, it is unclear what types of impact these consequences might have on this industry with concerns about the leadership pipelines are also unclear and should be explored. As a result, this section of the literature review was focused on leadership turnovers and its impact on organizations and on leadership.

For example, in Smith (2003) study on NCVs in California's community college, she found that over a third of these leaders left their institutions either directly or within a year after the vote. She inferred that more leaders may have left 
citing personal illnesses, family issues or retirement after the vote. She also noted that more leaders found it "difficult to survive a vote when it results from dissatisfaction with the [leaders] overall leadership" as compared to collective bargaining disagreements or strategic planning failures. Smith (2003) did not provide any specific data on where these leaders' careers after the vote, neither did she provide any follow up to why it was difficult to survive it and how leaders faired. (Schmidt, 2009) suggested that leaders "eventually exit after a period of resistance and denial" and they may do so under the guise that their departures were unrelated (para. 10). As a result, any mechanism that can limit or end the tenures of community college presidents is critical for investigation, particularly in light one of the most critical issues facing higher education industry today, which is the departure or turnover rates of college and university presidents in higher education (ACE, 2007, 2012; Shulock, 2002).

Leadership turnovers in Higher Education. More recently, the American Council on Education (ACE), and other scholars have also been assessing this wave of ostensible turnovers in higher education. Their findings asserted that such turnovers were indeed alarming and should be considered a critical leadership crisis that involved a massive turnover of the 'baby boomer' generation of presidents in higher education over the next ten years or so (ACE, 2007, 2012; AACC, 2005; Vaughan \& Weisman, 2007). Some of these organizations are tracking the ages and 
tenures of current presidents (ACE, 2007), while others take note of dismissals and turnovers reported to specialized agencies (Mize, 2011).

Similar finding were also noted in earlier studies, such as one conducted by Blum (1989), which presented a forewarning that turnover rates among different levels of administrators within higher education were rising. She asserted that the most crucial findings was the "twenty-four percent turnover rate[s] [were] found for administrators [annually]" in over 30,000 administrators in 3500 different private and public two- and four-year institutions (pp. A13-A14). Kirkland and Ratcliff (1994), using Blum's research, observed that such turnovers may be as high as $15 \%$ annually among community colleges executives and argued that more effective succession planning is needed to help limit the impact of these departures. However, these researchers have also advised that different institutions and specialized agencies appear to be addressing this problem by identifying and targeting key faculty, mid-level managers, and other potential leaders to close this leadership gap (ACE, 2007) while others suggest that such alarms may be unwarranted for organizations with succession plans in place (Kirkland \& Ratcliff, 1994). Closer analysis of these data and literature revealed that these researchers were focused on turnovers that were mostly voluntary. However, these researchers did not include the voices of leaders who experienced such events in their leadership roles; neither did these studies provide an evaluation about how these 
turnovers directly impacted or interrupted the organization, if at all. Thus, such situations are in need of further analysis.

\section{Leadership turnovers in California Community College (CCC) System.}

As previously noted, California's community colleges are struggling to close the leadership gaps in their presidential pipelines, and the impact of involuntary turnovers in the system is yet to be determined. However, the Community College League of California (CCLC), has been tracking turnovers and no confidence votes in the California community college system since 1998, and have suggested that such incidents are increasing and encourage drastic steps to reduce its impact.

For instance, one of the CCLC's earlier studies was designed to capture year-to-year comparisons of turnover rates among presidents, compare these rates to national averages, and then determine why leaders may be departing from these institutions (Mize, 1998). Using data on the CEOs in the 71 California districts at that time, researchers found that community college $\mathrm{CEO}$ turnover rates per year was at $13 \%$, slightly higher than the national average of $12 \%$ in community colleges (Mize, 1998). The study used the term CEOs to refer to those in executive leadership positions such as chancellors and college presidents. In addition, this study found that the tenure of presidents was significantly lower in the $\mathrm{CC}$ system, as few as four years, compared to the national average of up to eight years (Mize, 1998). The data indicated that the reasons for such departures were very diverse and 
often personal to the leader, but that most departures examined were based on retirements, and resignations of recent incumbents within a short time of being hired, and in some cases, based on those organizations that were simply in their cycle of change and did not renew contracts (Mize, 1998). Amidst the challenges noted earlier, the study indicated turnovers were also attributed to promotions and opportunities in the job market, as well as "changes in governance and administrative structures and processes may motivate CEOs to leave" (Mize, 1998, p.6). A key finding in this study is that turnovers in this system can be attributed to changes within the specific contexts of the institutions. Perhaps, these such turnovers were residual leaders from the pre-AB 1725 era who were also aging out of the system, and such changes would have required different types of competencies to meet the needs of the institution. One limitation of this study was that it did not capture the direct voice of the presidents as to why they left, but used information reported to specialized individuals such as boards, specialized agencies, as well as the media to capture the rationales for these departures.

More recently, the CCLC completed its biennial reports for 2011 on the tenures of California community college CEOs and found that, in addition to some of these aforementioned reasons for turnovers, some leaders do leave under pressure or "under-fire" (Mize, 2011, pp. 1, 3). Although Mize (2011) noted that the tenures of presidents remained within the four-year range and that retirement still accounted for most of the turnovers in CCC, she also noted that of the 138 
CEOs who left 72 districts between 2007 and 2010 , at least $32 \%$ of them left under fire. Mize noted that these types of turnovers were increasing, and she used the term "under-fire" to describe those leaders whose contracts were bought-out prematurely or not renewed, or those who left because of "problems within their districts" or organizations (p. 1). Although Mize's study did not explicitly outline if under-fire directly related to no confidence votes specifically, in a personal communication with R. Mize (July 27, 2011), she suggested that it more than likely included this category as well.

Factors resulting into leadership turnover. As previously noted in Chapter One, the scholarly literature asserted that turnovers rates of executive leadership throughout the United States and in several major industries have been on the rise for the last 20 years (Boeker, 1992; Wagner, Pfeffer, \& O'Reilly, 1984), while more recent studies have suggested that such turnover rates in different industries will be up by almost " $50 \%$...over the next four years" (Coyne \& Coyne, 2007, p. 62). In addition, Duffield, Roche, Blay, Thoms and Stasa (2011), suggested that there are many factors that could influence turnover rate among executives in organizations, and they found three main factors in the healthcare field, specifically. These researchers found that gender, age and educational levels were three of the most influential factors that could destabilize the healthcare system, particularly the nursing profession that is "largely dominated by females" 
(p.505). They also found that age as it related to retirement, as well as educational levels used in promoting individuals, were also factors in this process.

Shen and Cho (2005), in examining the factors leading to leadership turnovers, for example, observed that antecedent conditions could exist in the organization even before the leader takes over these organizations, and such conditions could include anything from in-group fighting, conflicts, or, in these cases, years of budget constraints or strained relationships with leaders. If not managed, any of these factors could result in a leader's demise. As a matter of fact, Smith (2003) confirmed that even when faculty indicated that several attempts were made to rectify such antecedent conditions or concerns within the college leaders and their boards were perceived as dismissive. As a result, the vote was the tipping point for many of these faculty members. Therefore, such antecedent conditions may be seen in the cycle of activities surrounding shared governance and communication (Schmidt, 2009; Smith, 2003), in addition to the contextual antecedent conditions factors within the organization, Shen and Cho (2005) suggested that there may also be antecedents within the leader's own self-efficacy, evaluations, and performance in their leadership, which they purport should also be examined to better understand the scope of these antecedent conditions and how they may contribute to the leader being ousted from the organization as well. Although these researchers focused on leadership situated in government and corporate America, they did provide several concepts and frames for understanding 
why leaders might leave their organizations and the conditions that were present that might have lead to these leaders' departures, which this study proposes may have similar implications for leaders in community colleges. As a result, the following section will examine the implications of leadership turnovers, the antecedent conditions that may result in these turnovers, and how this knowledge can be applied to leaders with a no confidence vote on their leadership.

Leadership turnovers as voluntary. The organization and management literature provided the most comprehensive concepts and descriptions of turnover, especially those related to executive turnovers, described as either voluntary or involuntary (Shen \& Cho, 2005; Wagner et al., 1984). Most scholars agree that voluntary turnovers are the more likely to occur when leaders depart from their organizations because of retirement, promotion, mergers, or resignation for personal reasons (Boeker, 1992: Harrison, Torres, \& Kukalis, 1988; Wagner et al., 1984). For instance, Wagner et al. (1984) suggested that such voluntary turnovers can be associated with the age of the leader and whether or not he or she has the type of capital needed to lead the organization. They also examined the age and size of the organization, which may have established succession plans as a policy; thus, allowing turnovers to occur when contracts end. These types of voluntary turnovers are also linked to well-established successions plans, and they seen as a type of 
"changing of the guards" or a natural process in organizational life (Harrison et al., 1988).

Other scholars suggested that executive turnovers can be less deterministic and involuntary for many reasons, including demotions and poor performance of the leader or organization; moreover, they may involve dismissals or firings, forced successions, or unplanned resignations (Coyne \& Coyne, 2007; Mize, 1992). Regardless of these rationales, scholars often focused on the interrelated links between the causes of such turnovers and the implications they can have on the organization when leaders exit. These factors may include the leader's overall performance in their executive roles (Frederickson, Hambrik \& Baumrin, 1988; Shen \& Cho, 2005), the leader's self- efficacy, competencies, or leadership approach (Coyne \& Coyne, 2007; Wagner et al., 1984) as well as the factors within their organizational environment, prior to and during the leader's administration (Harrison, Torres, \& Kukalis, 1988; Kirkland \& Ratcliff, 1994; Mize, 1998, 2007). Overall, these types of turnovers appear to have little to do with the flaws of the leadership and more to do with timing and the end of the leader's career.

Leadership turnovers as involuntary. Most scholars agree that the more critical of the two types of turnovers is the involuntary turnover, even in organizations with a well-made succession plan in place (Frederickson et al., 1988; Shen \& Cho, 2005). Such turnovers often include the leader being ousted from their 
institutions for any number of reasons, and such exits are regarded as being disruptive or abrupt or the result of a crisis (Coyne \& Coyne, 2007; Mize, 2007). Most of the literature defined involuntary turnovers quite similarly to the earlier work of Frederickson et al. (1998): a "dismissal...a situation in which the CEO's departure is ad hoc (e.g., not part of a mandatory, retirement policy) and against his or her will" (p. 255). Others who concurred with this definition have conducted research to explore the causes and situations that led to such actions, and the possible impact it can have on the organization and constituents (Coyne \& Coyne, 2007; Shen \& Cho; 2005).

The general consensus from these involuntary turnover literatures is that leaders can be dismissed regardless of the performance of the organization or that of the leader in certain situations and industries (Harrison et al., 1988; Fredrick et al., 1988). Shen and Cho (2005) suggested that dismissals can occur whether or not leaders felt like their leadership and leadership actions and performance were supported or constrained by their environments. These researchers and others found the leader's performance behaviors, and the organizations as possible predictors of these dismissals, but they too were also inconclusive. As a result, these researchers called for additional research about the causes for such forced exits or turnovers among different and similar organizations within the same fields and among different types of executives (Mize, 2007; Shen \& Cho, 2005). 
Data on these types on turnovers are often segmented by industries and sectors and they are often focused on either involuntary turnovers as opposed to voluntary exits. For example, Coyne and Coyne (2007) created a database to track executive turnovers between 2002 and 2004 in 1000 top U.S. companies by examining the tenures or employment statuses of current CEOs from year to year; this was followed by interviews with fewer than 20 top executives, including those recently appointed to executive positions. The purpose of their study was to determine patterns associated with how leaders entered and exited their organizations, as well as to track the next positions of these dismissed leaders. Using this knowledge, the researchers then asked current leaders how they survived in this environment and what lessons they had learned. Coyne and Coyne's (2007) findings indicated that among the subset of organizations examined, involuntary turnover rates have been rising annually among (a) CEOs already in place with longer tenures at a rate of $15 \%$, (b) newly appointed CEOs from within the organization at a rate of $17 \%$, and (c) newly appointed CEOs from outside the organization at a rate of $25 \%$. They also saw higher turnover rates among interim or "proxy" CEOs in these organizations (Coyne \& Coyne., 2007, p.64). These researchers then categorically described the what happened to leaders once they were involuntary ousted, such as their current career choices: (a) $65 \%$ of ousted CEOs were classified as "dropouts" within this two-year window, because "they no longer pursued an equivalent leadership position"; (b) $28 \%$ found similar or 
equivalent positions: (c) 4\% moved into a higher position, and (d) $3 \%$ experienced setbacks in their employments (p.65). The most significant and alarming data point here is that more than half of these leaders no longer sought after similar positions, essentially ending their careers at that point in this study. Perhaps, more longitudinal data is needed to see if any of these leaders eventually resumed these positions but from this data point, it does appear that these leaders were impacted by these decisions against their leadership.

Similarly, other agencies have collected data on the different forms of turnovers in non-profit organizations and in higher education institutions across the United States. For instance, in a recent Daring to Lead survey, researchers claimed that turnovers in non-profit organizations appeared to be increasing, and noted that, as of $2006, " 7 \%$ of leaders had turned in notices and another $67 \%$ were planning to exit," and that these percentages were slated to increase over the next five years (Cornelius, Moyers, \& Bell, 2011, p. 2). The causes for these turnovers also appeared to be diverse, including leadership burnouts, retirement, workload issues and conflicts in personal life (Cornelius et al., 2012). These researchers also suggested that these turnovers can also occur despite good or poor leadership performance evaluations and they can affect all types of non-profit organizations, including leaders in higher education (Cornelius et al., 2011).

In general, these researchers asserted that, in some organizations, the performance or lack of performance of the leader and the organization were critical 
factors in their dismissal, but not the only factors, and that such oustings can be survived. Coyne \& Coyne (2007) provided recommendations for surviving such outcomes emerged from the advice and knowledge of current CEOs in these organizations along, who would caution newer and junior executives from believing that they "stand a better chance" of escaping such a fate (p. 6). Although there were many limitations in this study, such as how leaders made sense of their dismissals and how these dismissals may have impacted them personally as wells as professionally, the inferences from this study were still insightful. For example, these insights included the voices of these leaders, and the impact of presidential successions on internal and external stakeholders, for example (Kirkland \& Ratcliff, 1994). Overall, the research found on turnovers were focused on leadership in the corporate or business sector, their assertions about such involuntary dismissals are not necessarily unique to these organizations, but they do have some implications for understanding other types of involuntary dismissals higher education as well. (Mize, 1998, 2007; Shen \& Cho, 2005).

Leadership turnovers as ceremonial. There was also a corpus of literature about what these types of turnover can mean for the leaders who experienced involuntary turnovers, and a few of them applied it to turnover caused by no confidence votes. For instance, March and Weiner (2003) suggested that NCVs were a part of the organizational politics, and can be symbolic, or act as a type of 
ritual that may be at work against the leadership. Still, other authors have asserted that such summations about the vote fail to explain why such ceremonies are still able to cause leaders to exit their institutions or can taint the reputations of these organizations (Shen \& Cho, 2005; Tierney, 2007). Still, a small cross-section of scholars have suggested that such actions in organizations are not necessarily related to the performance of the leader or that of the organization per se, but these involuntary turnovers can be ritualistic, such as scapegoating (Gamson \& Scotch, 1964; Pfeffer, 1977).

Scapegoating is described as a ceremonial process or mechanism that helps the organization demonstrate that they are implementing changes or adapting to the environment around them (Gamson \& Scotch, 1964), which, some suggest, can also happen even when the leader and organization are performing well (Birnbaum, 1988; Boeker, 1992). The earlier work of Gamson and Scotch (1964), although focused on the change leadership process in baseball, indicates that dismissals may sometimes have nothing to do with performance.

Using the performance of 22 baseball teams that were slated to have field management dismissed between 1951 and 1964, these researchers compared the performance of these teams two weeks before managers were dismissed and two weeks under new field managers. These researchers did not find any significant performance improvements after leaders were dismissed or under the new leadership in the first two weeks. They further theorized that, perhaps, there is a 
"ritual scapegoating" process that occurs when general managers and team owners "ceremonially" place the blame of the team's performance squarely on the leadership performance of field managers, which then allow these owners to fire and replace them with someone they believe will improve the team's performance (p. 70). These authors described this dismissal pattern as scapegoating, "a convenient... act in which the participants in the ceremony regard [it] as a way of improving performance" even without properly evaluating or evidence of such performance (Gamson \& Scotch, 1964, pp. 70-71).

Conversely, Boeker (1992), using Gamson and Scotch's theory, also examined 67 different organizations over two decades, and agreed that scapegoating did occur in organizations regardless of performance of the organization, but not necessarily among CEOs and executives alone. They asserted that such turnovers were likely to occur among mid-level management when the organization's performance was below average, and they asserted that such turnovers were more about "house cleaning" or "maintenance" than scapegoating (p. 416). In addition, they observed scapegoating among CEOs and other executives as "unique and infrequent" events that occurred in situations when the leader and organization's performances were poor, when the leader had little personal power especially among board members, and when the organization was also experiencing instability (pp. 416-418). Overall, they agreed that such dismissals are ritualistic because they 
are more about public relations and making changes on a surface level, than really addressing a specific problem.

Overall, the literature on leadership turnovers provided some knowledge about the leaders who have been dismissed or ousted and what were some of the consequences and aftermath of such actions. For example, the literature has shown that involuntary dismissals can be linked to performance or the lack thereof and it may be ceremonial in certain situations (Shen $\&$ Cho, 2005). The literature also highlighted the fact that turnovers can be highly complex and inevitable and suggested resources for presidents and institutions to support those in leadership. Similarly, the literature on leadership turnovers, particularly in community colleges, is increasing and attributed to performance, wherein leaders may end up leaving under of cloud of tension, such as leaving under-fire.

In summary, the literature review thus far has proposed that the tensions between what is expected and perceived in the practices and approaches of presidents, can lead to a no confidence vote crisis. In the same vein, the literature identified some of the structural, cultural and policy nuances than may be attributable to this surge of no confidence votes over the past three decades, which can have serious implications for leaders and these institutions. Admittedly, this literature review could have examined other concepts or rationales for the vote, such as abuse of power, unprofessional styles of leadership, abusive language and practices but there was little to no evidence that such activities were present in these 
cases or what were the reasons for these claims. More importantly, this literature review aimed to understand both the attributable factors and consequences of no confidence votes, such antecedent conditions or effects and aftermath of leadership turnovers, as well as to find out the possible impact of these sanctions on these leaders' overall leadership. In conclusion, there appears to be many factors that can contribute to these votes, including the factors present prior to the president's entry and these factors can be associated with the behaviors or actions of the president that, in turn, can induced a vote. Overall, the findings in the literature is inconclusive and was unable to pinpoint a specific action or trend that lead to a vote. In addition, this gap in the literature requires additional and in-depth research to understand the specific contexts and realities of these votes in different organizations and from the leaders themselves and how they make sense of these votes. Thus, the final section of this literature review will explore Weick's (1995) sensemaking approach, the main framework being used for this study to understand the impact of these no confidence votes on leadership.

\section{Theoretical Framework}

Scholarship on the impact of no confidence votes on leadership is virtually non-existent, and apart from one study and a few dissertations found for this review, little is known about the leader's reflection of this vote on his or her leadership, if any. In general, these very public votes appear to be used against leaders, with both 
seemingly good and/or poor performance evaluations, and they also appear to be based on perceptions and reactions to issues within the organization, rather than on reality (Schmidt, 2009). This writer stressed that the dominant narrative in the literature is still based on the rhetoric and meanings that the faculty, or proponents of the vote, used to enact the vote (Schmidt, 2009). As a matter of fact, Kuykendall (2010) suggested that one of the major reactions to the vote is to "never speak of it" (para. 1). She also suggested that this also brought with it a "veil of mystery about [this] under-analyzed phenomenon" which this study hopes to expose by speaking with participants who have experienced a NCV directly.

There were a few cases found where the voices of the leaders were reported. These leaders often provided sound bites about how they would respond to these votes, such as they were resolved to staying in their positions (Wright, 2007), or that their leadership was being scrutinized because they were trying to mobilize the organization and constituents did not want change, for example (MacTaggart, 2012; Schmidt, 2009). March and Weiner (2003) evaluated the impact of such leadership crises as causing "leadership blues" in one respect, but also posited that such mechanisms are also "slash and burn tactics" which are part of this political posturing between the leaders and the embittered faculty (p. 6and 8). They further posited that such methods can be disruptive to other constituents (i.e., students, staff and other outlier faculty), whose voices sometimes get "lost in the campus politics" as well (p. 8). These researchers also found that leaders described leadership blues 
as emotional, such as they were feeling "full of outrage and hurt... [or felt] far more pain than they bargained for" (pp. 8-9). Similarly, it is unclear if leaders would face backlash in their careers if they were to express such angst or anxiety about these actions, and whether or not such openness about the votes could be detrimental to them and their presidencies. However, one theoretical framework used to deconstruct such realities is proposed in Weick's (1995) model of sensemaking. This model suggests that individuals generally try to make sense of who they are and the realities in which they lived in by using seven different dimensions in their constructed communications about their situations (Weick, 1995). In addition, several researchers have used this framework as a vehicle to help individuals tap into these difficult experiences in their lives and will be discussed in more details in the following section.

\section{Weick's Sensemaking Theory}

Weick (1995) defined sensemaking as simply "making sense" of ourselves and the context in which we exist (p.4). He described his theory as resting on the recipe of "How will I know what I think until I see what I say?", a recipe for making sense of our crisis situations, and then embarking on creating or "authoring" their own sensemaking (p.7). The theory posited that sensemaking begins with sensemakers, as they frame and reframe complex events and incidents that they experience in a local crisis situation (Weick, 1995). To do this, individuals are 
expected to take some time to make sense of their situation and to reflect on their experiences. Although no specific timeline is offered as a formula, the inferences from Weick's (1995) previous study design suggests that periods of reflection may be as much as 10 or more years, seen in the Mann Gulch study.

Weick's (1995) framework also outlined that certain elements should be examined in the ways individuals make sense of their situations, such as examining their:

- Identities, meaning who they are and what they think about themselves and their leadership;

- Situations, retrospective considerations about the critical (positive) or negative incidents in their lives, especially as it relates to no confidence votes;

- Conditions, how they enacted behaviors in their environment;

- Interactions, understanding of the social or social interactions, including communication in the events,

- Reflections, ongoing reflection about these incidents, (f) signals and extracted cues, and

- Rationales, plausibility about what happens emerges and aligns with sources from other texts, as seen in Figure 9. 


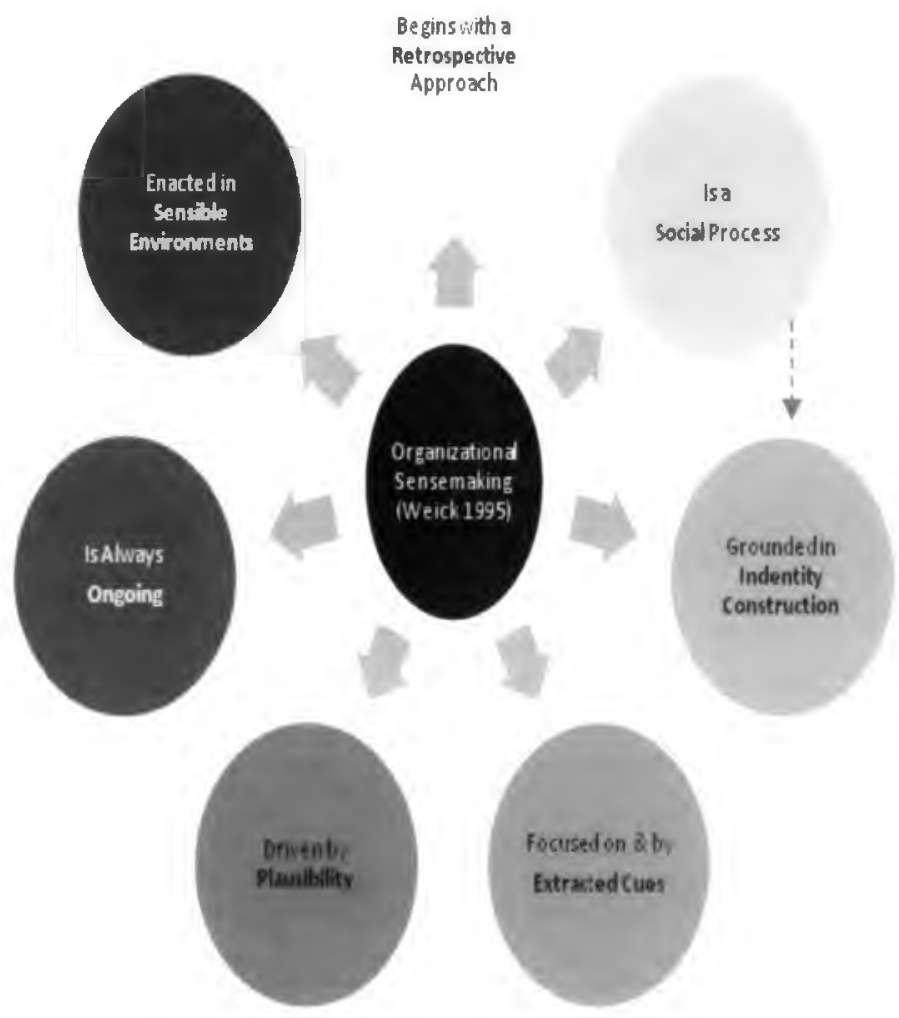

Figure 9. Conceptual Map of Weick's (1995) sensemaking approach. The figure highlights the seven elements of sensemaking and how the social aspect of sensemaking also connected and informs identity constructions. Retrieved from http://cmapspublic.ihmc.us/rid=1210136295281 565567225 I 1239/Organisational \%20Sensemaking.cmap

Weick, Obstfled \& Sutcliffe (2005) also discussed the importance of leaders being reflexive during times of uncertainty and ambiguity to understand our environment carefully and to not distort what we are seeing. Weick (1995) further 
posits that individuals are forced to make sense of their environments when shifts or changes occur; more specifically when there are too many unknowns or uncertainty, or too many possible meanings, too much ambiguity in the environment. Such reflection is particularly important in institutions that are described as socially constructed entities, reproduced within all social institutions in American society, wherein leaders are required to perform their roles (Acker, 1990, 2000; Birnbaum, 1989; Bowles \& Gintis, 1976; Lahtinen \& Wilson, 1994; Rosser, 2003). Yukl \& Becker (2006) also suggested that leadership is a constructed reality wherein leaders are often framing the needs of the organization to mobilize others to get the work done. Eddy (2005) used this approach to help her CEO participants open up about critical events (both positive and negative) in their leadership, finding that each participant found it less threatening to discuss such events, and were better able to reflect on who they were, their overall leadership, and interactions with constituents during critical events in their presidencies, when asked to make sense of it.

Weick's (1976) earliest application of this theory was previously applied to the educational context, wherein he examined the ways individuals interacted with each other, in what he referred to as a loosely coupled system. As previously mentioned, such loosely coupled system refers to the ways different groups or divisions of labor are organized around their work and connected across the academy as these groups have to interact or communicate with each other. For Weick (1976) loose coupling is also about finding ways to connect to each, 
understanding each other and coordinating activities to fulfill the institution's purpose. However, during times of uncertainty or ambiguity, it is possible that such lines of communication can become interrupted (Weick, 1993), distorted (Weick, Obstfeld, Sutcliffe, 2005), or involve some form of manipulation through sensegiving (Smerek, 2011) that may have serious implications for leaders if not recognized and dealt with early.

\section{Opposing views to Weick's sensemaking approach. Critics of Weick's} (1995) sensemaking approach argued that sensemaking only explains the outcome but what is critical in these situations is sensegiving (Gioia \& Chittipeddi, 1991; Smerek, 2011). These scholars examined sensegiving and sensemaking cycles between university presidents and their constituents during certain activities. For example, Gioia and Chittipeddi (1991) studied the cycles of sensemaking and sensegiving between a president who wanted to enact his strategic planning process and the many interactions that occurred between him and institutional players regarding the meaning, symbols and signals being inferred by his plan. These researchers describe sensemaking as the "meaning construction and reconstruction" of the parties involved as they attempted to develop meaningful frameworks for understanding the intended strategic changes proposed by the president (p. 442). As the leader scaffolded portions of his plan, institutional players were concerned about whether the president was valuing and being responsive to the culture, symbols, and traditions of the institutions, which they describe as the "currency of 
exchange" as groups interact and communicate in organizations (Gioia \& Chittipeddi, 1991, p. 446). As the process continued, Gioia and Chittipeddi (1991) asserted that both the leader and institutional players were engaged in a sensegiving pattern of communication, which is a process for "influencing the sensemaking and meaning construction of others towards the preferred redefinition of the organization reality" (p.442). Thus, this growing ambivalence and equivocality (a mixture of ambiguity and uncertainty (Weick, 1995) then caused the leader to slow down in responding to this equivocality, thus giving sense and making sense at the same time. This assessment of sensegiving is useful for understanding what can influence our lens and ultimately persuade our decision-making process within an organization.

Likewise, Smerek (2011), suggested that sensemaking requires closer examination of power relationships in organization that can influence the politics of sensemaking, which requires negotiating how presidents and their constituents interact over time. Smerek (2009) suggested that sensegiving is more than individuals wading through limitless amounts of interpretations and meanings, defining it as "the internal process of influencing a target audience's perception of meaning" (p.144). As a result, individuals in the environment can and will develop their own frames and meanings about what is going on, sensegiving, in response to any interruptions or changes in their environments. Such sensegiving, he posits, depends on the relationships between these groups, thinking or mindset of the 
individuals in this setting, it is unclear what the implications can for leaders leading these groups (Smerek, 2009).

Still, Cantos, Neidig and O'Leary (1993) suggested that such collective mindsets and interaction can have both positive and negative implications, particularly as it how it is related to attributional biases. Cantos et al. (1993) described these biases as occurring when individuals apply their perceptions and beliefs about others behaviors as the cause for their reaction. These researchers observed these biases among individuals involved in domestic violence, where one individual placed blame on their significant other for their reactions to their behaviors. As a matter of fact, during such crisis moments, one individual may perceive his or her actions as more appropriate when compared to the other, a type of self-serving bias as well. Concerning no confidence votes, it is possible that both the sensemaking and sensegiving process of the leaders and faculty can be influenced by such biases and expectations we have about others, as asserted by Burgoon (1997). Meaning, faculty when faculty suggested that they gave the vote because of the leader's poor performance and they had no other alternative to act, then this basis is observed. Likewise, when leaders perceived that they got the vote because they were indeed trying to move the organization forward but were faced with resistance from the faculty, this too can be seen as a type of self-serving bias. As a result, the two narratives about the rationales for a no confidence vote previous noted in Chapter One also appear to align with this arguments that, perhaps, both 
the faculty and the leaders in these incidents may be making sense about the other's actions through these types of biases or expectations. As a result, such perceptions and expectations can be fueled by biases (see Heider, 1963), which refers to the assumptions individuals may have about or attribute to others, and these individuals may not have sufficient or accurate information about what the behaviors they are observing. As a result, this study will also include some of the literature from this field and others to better understand the phenomenon being examined.

Overall, Weick's (1995) sensemaking theory is focused on the constructed narratives and interpretation of the sensemaker. Such research does not guarantee that leaders will be purely objective, truthful or open about their experiences and it possible that individuals who attempt to make sense may still be scripted to a certain extent. This may be true for leaders in this study, whose narratives will be triangulated with additional narratives found in written text, with the hope that such additional insights will provide a fuller story about a phenomenon. Additionally, by using the seven dimensions in Weick's (1995) sensemaking approach to conduct an analysis, this study hopes to capture the ways leaders (a) construct their identities; (b) retrospectively consider the critical (positive) or negative incidents in their lives; (c) enact behaviors in their environment; (d) conduct social interactions, including communication; (e) reflect on these incidents, (f) extract cues, and (g) determine the plausibility about what happens, emerges, and aligns with sources from other texts. 
Weick and leadership identities. As previously mentioned, leadership identities have been used key construct examined in a plethora of studies. Weick (1995) theorized that identities describe the ways individuals make sense of who they are, ask questions about who they are, and what they think about themselves and their leadership, for example. While there are other competing theoretical frames that this study could have chosen to discuss leadership identities and how leadership is developed, Weick provided a framework for analyzing each dimension in his theory, which has been cited over 17,574 times, according to GoogleScholars.com.

Weick's sensemakers. Weick (1995) maintained that sensemaking begins with and is centered on the sensemaker in an organization, in this case, the leader. These sensemakers generally extract information from their environments to make sense of the interruptions and unique and transitory experiences present in their organizations or environments. Starbuck and Milliken (1988) described this extraction process as a type of filtering of different stimuli and responses in the environment that the sensemaker or leader may notice. Weick (1995) asserted that individuals garner information from their environments through their interactions with others in these contexts and by using the "the substances of sensemaking" (p. 106), such as words or languages used to convey information about the environment, how it is structured, how it works, its ideologies, and what is expected. Individuals then frame meanings, and then select and label information 
from their environments about what they have observed. Then they extract cues and interpretations, by using other familiar macro-level frames (i.e., ideologies, data); premises (i.e., third order controls or assumptions), and socialized ways of thinking or paradigms (i.e., exemplars, illustrations, collection of knowledge) to create sense out of what they have observed. Once individuals begin to share and gather other sensemaking from others around them about what is going on, these individuals begin to develop consensus about what is going on. In essence, individuals are usually looking for symbols, rituals, ceremonies that they try to connect with and connect to situations as a way to make sense of them and use these explanations to get them back to stability. Thus, members of organizations will select information from the institution's context and norms for doing things, such as traditions or traditional ways of acting. Then such members begin to draw inferences from plausible stories that they remember and share, which then become maps or sequences and experiences such as lessons we have to guide our processes in organization and unknown situations.

Starbuck and Milliken (1988) suggested that failure to understand such relationships and to observe changes within the environment can lead to blind spots. They described such blind spots as occurring when "we think we have seen something often enough to filter out situations" but such filtering might be a key spot for distorting our view of what is really going on (p. 38). For example, Weick (1988), in his assessment of the Mann Gulch fires, an unpredictable fire that 
claimed the lives of several smokejumpers, also suggested that such blind spots prevent us from seeing what we don't know about situations. He refers to such blind spots as the "fallacy of centrality" (citing Westrum [1982]) which exists when individual believe that if situations were indeed changing or different around them they "would have known about it, but since [they] don't know about it, it doesn't exist" (p. 370). Such overconfidence about our organizations and the possible shifts, uncertainties and ambiguities that can occur and generate ambivalence across groups about what is going on, can be serious danger for leaders who will lead organizations. Furthermore, Weick (1995) asserted that sensemaking is also about making plausible judgments and decisions about what is going on in our environment, not necessarily alone, but with input from others in our organizations. Figure 3 presents a conceptual map of Weick's (1995) sensemaking approach to organizations.

Why use a sensemaking approach. Other theoretical frames were considered for this study and included leadership theories but offered no framework for analysis. As previously mentioned, Bennis' (2004) seven ages or stages of leadership development, reviewed earlier, is useful for delineating ways in which leaders can examine their self-efficacy and leadership-efficacy in their respective roles and throughout their leadership, but it does not provide a premise for assessing or analyzing these frames among leaders. Similar theories were also considered, 
such as Dreyfus' (2004) five-stage model of skill acquisition, also used in other fields, like nursing, to describe practitioner's skill development (see Benner, 2004). However, these theories either implied some linear understanding of development or did not provide a proper frame for exploring the ways they think about themselves and their leadership, compared to Weick's (1995) framework.

Overall, this approach is consistent with the overall design which is to examine the ways presidents make sense of no confidence votes, their leadership and leadership practices within the context of these critical incidents and events on their leadership. In addition, other researchers have used this theory to examine this phenomenon and will be discussed in greater details below. Furthermore, this approach not only flows logically from the problem being investigated but it fits well with the overall design. Therefore, by using the dimensions described in Weick's (1995) work, it may be possible to investigate the origins for these no confidence votes' patterns and mindsets that lead to them, especially in the narratives of the leaders and how they make sense of who they are as presidents. The following section provided additional evidence of how this theoretical framework was used in dissertations about no confidence votes in higher education.

\section{No Confidence Votes Dissertations using Sensemaking}

The first dissertation was conducted by Leinbach (1997) who completed a two-year study at an American community college, where a leadership crisis was 
unfolding as a result of three no confidence votes on the president and his administration within a two-year window. The researcher used a case study approach and conducted focus groups interviews with thirty-four respondents from the college, including clusters of individuals from the board, faculty, staff, student leaders and the presidents, to learn more about their perceptions of what caused the vote (Leinbach, 1997). He outlined the salient allegations and failings of the leadership as perceived by the students, staff and faculty, and other administration, such as lack of attention from the leader to "sexual harassment complaints from students against the faculty," "administrative pay raises while there were no faculty raises," and "non-renewal of tenure track" positions, amongst other challenges facing the organization (Leinbach, 1997, pp. 170-192). In addition, the researcher noted that, after a period of great pressure from the faculty and union, the Board felt they had no other choice and decided to force the president, Dr. Smith, entered into early retirement. Dr. Smith also agreed that he lost the support of the board and the trust of the faculty, and felt like he had been "attacked by the faculty and media" (Leinbach, 1997, pp. 209-210). Overall, the president, Dr. Smith, acknowledged that he had made mistakes as a president; however, his perception of the vote was that it was "bogus" and highly personal, especially because he felt like he eventually implemented programs and solutions that would have addressed the concerns of constituents over time (Leinbach, 1997, p. 210). He further qualified his statements by noting that the local newspapers "refused to listen" to him or the 
board about specific details in the case but appeared to err on the side of the faculty and union and deemed their narrative as factual (Leinbach, 1997, p. 209-210). As a result, this leader felt constrained by the vote, and limited to act and perform his responsibilities, especially after he believed he had lost credibility with both internal and external communities, and therefore, opted to retire after a third no confidence vote against his leadership occurred (Leinbach, 1997).

Leinbach's (1997) dissertation provided keen insights about the president's experience with the vote as well as the sense-making, perceptions, and additional narratives other constituents were provided with attention to the no confidence votes at this community college. The narrative or counter-narrative of the president, Dr. Smith, is one of the few instances where the leader outlined the effects of the vote on his leadership, although he also appeared to be at the end of his professional career, although this retirement coincided with impending third vote. However, this study and others failed to address the impact of the votes on leaders who were not nearing retirement from the presidency; neither did it demonstrate if the faculty concerns were substantiated or resolved as a result of the leader's departure, a gap which this research study expects to fill from the narratives of presidents in community colleges. The other two dissertations (Estepa, 1997; Schepker, 2012) and thesis (McKinniss, 2008) that were found also investigated no confidence votes or leadership dismissals within the context of the organization, and included corporate and the university or college settings, with close attention given to the 
unique sensemaking that both the faculty and presidents made of these sanctions on their leadership.

The second dissertation was a study completed by Estepa's (1997), who examined the factors that precipitated the vote at a Catholic University between 1988 and 1993, after the President Smith made changes to governance structures. Using the Miles and Huberman (1996) quasi deductive procedure, where the questions are derived from the chosen research conceptual framework or theory, Estepa (1997) found the following factors as leading to the vote: 1) Adversarial relationships between faculty and the president; 2) A history and culture of strained communication between faculty and president, 3) pressures from external agencies, and 4) Other personal factors such as perceptions about the leader's inexperience, ego and overall leadership ability. Estepa (1997) suggested that the faculty, who believed in the values and rich traditions of the institutions, saw the vote as means for venting their frustrations against the leadership. President Smith received the vote in 1992 and would resign in 1993 . He would go on to defend his experience as a leader and suggest that the faculty had an inability to listen and that he got along with most people. Although this researcher did not use Weick's (1995) sensemaking approach in his study, he infer that certain events and factors can precipitate a vote against the leader. In addition, this study also highlighted the type of contextual factors and how quickly they can change to support or reject the leadership in these institutions. 
The third dissertation was conducted by Schepker's (2012), who completed a two-phased study focused on the implications of NCVs on CEO corporate leaders. Using 3,648 firms and $1080 \mathrm{CEO}$, the focus of this investigation included the perceptions of board about leaders and their capabilities, as well as what happens when boards lose confidence in their CEOs. Using Weick's (1995) external cues as a premise for his study, Schepker (2012) found that there were many factors that can shape the perceptions of these boards. Starting with antecedent factors such as poor economic times; to performance rationales such as poor performances seen through profit and losses, personality differences, disagreements about fiduciary duty to stakeholders, to personal rationales, such as a lack of human capital on the part of presidents or lack of experience, all of the factors are cues that boards use to evaluate their CEOs and begin to lose confidence in them. Such evaluations can then lead to a dismissal of the leader and the aftermath of such action can be different for stakeholders, who may be relieved by the actions or the board, as well as failure for the $\mathrm{CEO}$, whose reputation may be scarred by these actions against them. As a result, Schepker (2012) proposed that for some of these leaders their careers were often stunted and many of them do not find or pursue similar positions, especially for those leaders who were dismissed for poor performance, for example. He also mentioned that many of these CEOs were left to deal with a stigma of being ousted during their careers. 
Finally, McKinniss' (2008) thesis was also examined, and he used three NCV cases in three different institutions, Baylor University, Texas A\&M University, and Goddard College to evaluate no confidence vote events. Using articles from different trade journals and newspaper reports, McKinniss delved into circumstances leading to the vote. The key rationales for these votes were based on shared governance violations. In the vote at Baylor, the president, Dr. Robert Sloan, was believed to have worked in an environment void of trust and filled with anxiety, particularly after the faculty thought the president had disregarded their "input and role in governance" processes (p.33). So much so that when Dr. Sloan attempted to mobilize the organization toward change, certain faculty member deemed his actions as moving too quickly and that their voices were not heard or vetted. McKinniss further asserted that communication between the faculty and president was strained and bitter. Dr. Sloan would later resign a year later and McKinniss (2008) noted that the president thought his departure would allow for healing to begin after long periods of stalemate between himself and the faculty. McKinniss (2008) also mentioned that Sloan had the board's support, but he still resigned, and that not all faculty were in support of this vote. Similarly, McKinniss (2008) suggested that President Juarez, at the Texas A \& M University, received the vote after he attempted to change the tenure and promotion policies in 2004, another shared governance violation. Although President Juarez contended that he was able to implement the policy with faculty input and support, the faculty senate 
"disagreed" and suggested that the plan was being "implemented without regard to the senate" (p. 40). A year following the vote would result in the president citing "a lack of cooperation" from the senate and would move to disband the senate and to develop a new senate constitution. This action led to serious outrage against Juarez and the board, and Juarez would soon after retire stating that he needed to spend time with family.

In McKinniss' (2008) third case study of a NCV at Goddard College, wherein President Richard Greene worked in a highly collaborative environment, with strong decision making processes, even to the point of choosing a paint color for university buildings. The NCV resolution emerged out of a financial crisis where the faculty blamed the crisis on the president mishandling of funds. In an effort to defend his actions, McKinniss (2008) noted that the president trumped up these concerns to "distrust... in normative processes" and the vote as a type of "clichéd strife" (p.48). Soon after, the faculty also drew attention to the leader's autocratic style of leadership as problematic and called for his resignation, which he later provided.

Altogether, McKinniss' (2008), Schepker (2012), Estepa (1997) and Leinbach (1997) each provided a wider context for understanding these situations that can shape the factors leading up to a no confidence vote, and some insights about the implications and consequences of such actions against the leadership. In addition, these studies provided direct and indirect access to the voices of these 
leaders and their initial reactions to these votes or sanctions against their leadership. For example, McKinniss (2008) used Weick's (1995) sensemaking approach to analyze the ways in which both the sponsor's of the vote and leaders made sense of the actions and behaviors of the other in highly ambiguous situations and created meanings from these perceived behaviors and patterns. A major criticism of McKinniss' (2008) study, however, is that most or all of the data in the study were gathered from trade journals and newspaper articles or secondary sources. It is unclear if this researcher obtained or used any primary sources, and the voices of both the sponsors of the vote and these leaders were limited to these sources. The research analysis in these studies focused on the necessity of the actions against the leadership, and in some cases appeared legitimate; however, these studies provided limited insights into the immediate, distal, or direct effects of the votes on the leader targeted in the organization.

Overall, the empirical literature on this phenomenon is scarce, and the literatures that were available are often focused on the narratives of the sponsors of vote. As result, little is still known about the leader who experienced these votes and how they survived the aftermath and consequences of the vote. Two of these studies also used Weick's (1995) sensemaking model or theory to delve into some of the antecedent conditions and meanings that leaders and constituents gave concerning the votes in their organizations, which included some of the same sentiments around leadership skills and communication, as noted previously. A 
major observation about the case studies is that Weick's (1995) sensemaking approach called for retrospective sensemaking, wherein leaders were asked to reflect on their actions leading up to the vote. As a matter of fact, the responses of the leader appear highly defensive and were often captured in the early moments of the ordeal. Hence, it unclear if such retrospection would have changed over time when leaders may have been far removed from the situation and have had some time to think and reflect on these votes. Thus this study proposes that additional research is indeed needed to shed light on these sanctions, specifically against California's community college presidents and to ask them to make sense of these NCVs against them.

\section{Conclusions}

In summary, the objective of this literature review was to determine some of the possible impacts of these votes and their influence on the leader and the organization, which appears to be vast and varied. The literature review offered many variables and considerations for understanding these votes, the situations, antecedent conditions, and contextual factors that can lead to these votes. This study is not proposing that such votes are bad, good or undeserved; however, this study is proposing that the uses of these types of actions may be increasing, at least in one community college system, and that more research is needs to understand how these votes can impact the leader as well as settle the concerns of the sponsors 
of the vote. Overall, this literature review also provided alternative points of views for understanding why faculty may use the no confidence vote options, particularly when the need to interact and make judgments about the leader's performance and responsibilities. As the literature indicated, the perceptions of sponsors may be influenced by many factors and may or may not be accurate evaluations of these leaders, and some researchers proposed finding alternatives to these votes (Tierney, 2007). As noted earlier, the literature outlining the implications of these votes on the organization and the leadership is fundamentally sparse. As a result, this literature review delved into three major reasons why institutional players may move for a vote, which include: poor leadership or leadership style and performance, violations around shared governance, and a lack of communication. Similarly, this research also identified three main implications of the vote on leaders and institutions to include: (a) possible repercussions for the organizational players and leaders, (b) impact on the reputations of the organization and leader, and (c) the consequences of the aftermath that could include leadership turnovers and irreparable relationships within the organization. Such implications, of which some are anecdotal, can further compound some of the existing problems or challenges already present in the organization and may have more far-reaching impacts on its constituents and the stability of the organization, than this review of literature can explore in this space. Still, administrators and their constituents may need to consider the causes and implications even to determine what steps may be needed to 
evaluate their leaders and provide equally effective alternatives for drawing attention to the leader's behaviors and actions in the organization. Perhaps, boards may need to play a more pivotal role in this process, as well as help to develop processes that will prevent unwarranted dismissals, under these sanctions. Overall, this study asserts that the first place for analysis begins with the leaders and how they experienced these votes during their presidencies is crucial for understanding this phenomenon. 


\section{Chapter Three}

\section{Methodology}

The primary purpose of this phenomenological study was to explore and deconstruct the potentially rich narratives of presidents who experienced a leadership crisis, as a result of vote of no confidence from their respective community colleges. According to Bogdan and Biklen (2007), a phenomenological study method should be used to obtain the meanings and points-of-view about a particular situation that only key informants have experience, "letting them teach you" about the importance of their experiences (p. 35). In addition to exploring the counter-narratives of presidents who experienced a leadership crisis, within the community college environment, this study is also positioned to expose the possible implications and consequences of the vote on their leadership.

\section{Research Questions}

The main research questions guiding the study include the following: How do presidents make sense of a no confidence vote on their leadership?

What factors do they attribute to the vote?

What lessons do they learn from these crisis events, if any?

\section{Research Approach}

This study's phenomenological approach has an embedded interpretive research paradigm, a paradigm that allows participants to subjectively construct 
their own realities and give their own meanings of the phenomenon they've experienced (Creswell, 2007; Ormston, Spencer, Barnard, \& Snape, 2014). Creswell (2007) explained that such studies allow the researcher to gain "a deep understanding of a phenomenon" from the individuals who have insight about it, which can better explain what individuals in that group have experienced (p. 62). In other words, phenomenological research allows participants "to relive in their minds the experiences they have had" about a situation (Fraenkel \& Wallen, 2008, p. 429).

This phenomenological approach is not without drawbacks, however. For example, a major challenge of this research design is failing to obtain the story as a means for understanding the phenomenon for any number of reasons, including selecting participants who have not experienced the phenomenon (Creswell, 2007). As a result, this researcher is quite cognizant that these leaders may not be as open and transparent about their experiences and may censor their thoughts as well as come off as being scripted in what they share about their experiences (Hochschild, 2009). To reduce this problem, this study carefully selected participants who received a vote of no confidence, began building trust with each participant by being transparent about the study and each process as outlined in the recruitment process (see Appendix A, p. 489). In addition, this study focused on framing and asking the right questions, a process supported in literature (Creswell, 2006; Fraenkel \& Warren, 2008). Thus, this study's investigative process is designed to 
provide insights into these narratives or stories of a purposeful sample of four (4) presidents and chancellors from the California Community College system, asking them to construct their narratives or counter-narratives to describe their lived experiences with the vote.

\section{Research Framework}

Being ousted from a leadership position is quite possibly one of the more difficult events anyone can experience during his or her career. In addition, even if leaders don't leave, it is unclear how they will situate their leadership within the institutional context, knowing that there are members within the organization who may be dissatisfied with their leadership. One researcher proposed to understand this type of framing, investigating the insightful counter-narratives or counterstories that "members of an outgroup [outsiders in their own group]...tell themselves and others... [about their] 'counter-reality'... [or a] plausible 'counterview' of what happened," especially during a crisis event (Delgado, 1989, pp. 24342435).

Thus, the aim of this phenomenological study is to move beyond the masternarratives that hinge on descriptions of idealistic leadership styles, approaches, or tasks in the academy; instead, the goal of this study is to engage in an inquiry of counter-narratives with presidents about a specific leadership crisis (Eddy, 2005). This type of research approach is a valid mechanism for capturing the meanings 
individuals give to their experiences, and for exploring and evaluating these experiences and the worlds that they live (Denzin \& Lincoln, 1998).

To accomplish this goal, this study used the seven elements of Weick's (1995) sensemaking approach as the key instrumentation, to help leaders tell their stories about no confidence votes on their leadership. A major intent of this investigative process was to first ask these presidents to construct their leadership and leadership practice in a shared governance system, using frames found in the leadership literature (e.g., Bass, 1990), especially as it related to the organizational cultures within the academy (Birnbaum, 1988), and how leadership was structured, shared or distributed within organizations (Spillane et al., 2004). The secondary intent is to have leaders share the factors that lead to their votes as well as the aftermath of the vote. To accomplish these objectives, this study examined the reflective stories, consciousnesses, and interpretations of these events in the narratives of the leader, by exploring the seven properties found in Weick's (1995) sensemaking approach in a crisis.

Weick's sensemaking approach. Weick's (1995) sensemaking approach is being used as the theoretical framework and diagnostic tool for understanding how leaders make sense of these votes on their leadership in California community colleges. Weick $(1995 ; 2001)$ suggested that individual sensemaking involved these leaders reflecting on what they experienced, particularly during a crisis event, 
and how such leaders frame and extract meanings and cues from it as they tell their story. Weick (1995) liken such stories to a map or guide that individuals create about the way they lived an experience and a guide that anyone can use to help them similar situations.

Weick's (1995) analytical approach is not without its critics, however (see Basbøll, 2010 and Weber, 2006). One of Weick’s (1995) fiercest critics, Basbøll (2010), proposed that Weick not only "plagiarized" most of his concepts about sensemaking, but that he also plagiarized the Alps-map story from "Miroslav Holub (1997)" to make his point about the knowledge we can glean from even old stories or roadmaps to survive a crisis (p. 116). He also likened Weick's approach to that of poetry, because he accused him of both plagiarizing from poets, such as Dickinson, and for not using his own empirical work to back up his theory (p. 166). Weick (2003)did respond to similar and previous allegations about the usefulness of his theory, wherein he recanted his earlier empirical and analytical he did including the Mann Gulch disasters and other research he completed to support this theory.

Other researchers have found this approach useful when working with leaders in crisis, as noted in Chapter Two. For example, Eddy (2005) found this approach useful with her study with community college presidents. In her research with nine community college presidents, Eddy (2005) used this construct to help leaders open up about critical events in their leadership. As discussed previously, Eddy (2005) found that leaders were able to identify a) the mental maps and guides 
they used in defining their leadership identities and their decision making processes; b) how they defined their work and overall performance, and c) how leaders constantly changed and adjust their conceptions of leadership overtime. Overall, in her sample, leaders found this process less threatening to discuss such events, and were better able to reflect on who they were, their overall leadership, and interactions with constituents during critical events in their presidencies, when asked to make sense of it.

By using Weick's (1995) approach, this study examined the impetuses for such drastic actions in the academy, using these leaders' experiences and sensemaking, to understand what they recalled as the actions they took before and after the vote, and how these highly public referenda have impacted these leaders' roles and their credibility, especially with stakeholders, constituents, and the overall institution (Schmidt, 2009). A major theoretical assumption in using Weick's sensemaking approach is that leaders have taken the time to reflect on what they have done and able to create a plausible story about these votes. Thus this study is assuming an interpretivist stance which has a social constructivist premise, where the research will allow us to examine the world we live in with its multiple meanings (Bogdan \& Biklen, 2007).

With this in mind, Weick's (1995) sensemaking theory appeared the most valuable theoretical approach and analytic framework to capture the narratives of these presidents. One expected finding in this study is that leaders may have similar 
sensemaking stories as previously discussed in other studies and dissertations outlined in the literature review, such as leadership denial about the reasons they got the vote as against their leadership. One way to minimize this potential threat in this study is to triangulate these leaders' narratives with reports in the media and in other documents, and to ask these leaders to respond to the rationale or sensegiving of constituents in these reports.

\section{Participants}

This exploratory design consisted of a purposive sample of three to five presidents from California community colleges who experienced a vote of no confidence. The initial pool of potential participants included a list over fifty presidents in the California's community college system who received a vote of no confidence between the mid-1990s to the present. This list came from several sources. For example, each institution that delivered these votes to their leaders is generally required to report this information to the Chancellor's office and to the Community Colleges League of California (CCLC). Until recently, these votes had to be reported to the CCLC until recently; however, the CCLC is no longer required to collect this data but are now focused on leadership tenures, which could include no confidence votes (R. Mize, personal communication, July, 26, 2011). In addition to these public reports, the Academic Senate meeting minutes, newspaper briefs and other documents are generally public and available online for some of these participants, allowing the researcher to identify the individuals who received 
this motion. A desired sample of three to five community college presidents were identified and recruited, using specific parameters needed for the study.

Attempts were made to contact all key informants, who were unknown to the researcher, and all contacts were made via email using the public directory from the Chancellor's office and other newspaper and internet searches, such as Google searches. These emails included an electronic letter describing and recruiting participants for the study (see Appendix A, p. 489), as well as a letter from the department authorizing this study (see Appendix D, p.499). Thereafter, five potential participants were contacted and, of the five, only four participants agreed to participate in the study and completed consent forms. Each participant in the study was selected using the parameters described below, and asked to complete and return a signed consent form (copies will be sent to them) to finalize their participation in the study (see Appendix B, p. 493). Moreover, each participant must have been 18 years and over, and have a working knowledge of the English language, such as being able to speak, write, and listen in English, to participate in this study.

Overview of demographics of each participant. This phenomenological study obtained the in-depth narratives of a purposive sample of four participants, Bill, Susan, Paul and Debbie (pseudonyms), two men and two women. All four participants satisfied the criteria outlined in this study and noted in the IRB 
protocols (see Appendix H, p. 507). In addition, each participant had been a leader (i.e., president, chancellor) over a single community college or multiple colleges during his or her tenure as a community college leader, and, collectively, these participants present over 120 years' worth of experience in higher education, including in small, medium to large community colleges and universities. The average tenures of each of these leaders was about 30 years, with Susan serving over 38 years, Paul has over 40, Debbie has about 27 years of service and Bill over 30. At the time of these interviews, some of these leaders were either currently retired or still working in the CCC system or other systems in varied capacities.

Overtime, these leaders have also accumulated numerous accolades, recognitions and awards throughout their careers for their services in higher education and have led institutions that are prototypes of success in different areas, during their tenure and after. All of these participants talked about the sacrifices they made to be a leader, including working late nights and weekends, lack of privacy, prolonged separation from family and spouse for work, dealing with difficult situations and problem solving. In essence these leaders talked about the courage one needs to lead, especially in today's environment for constant fundraising, budget cuts, and meeting the growing needs of students and employers. Each participant talked about personal identities, professional development, and career trajectories as a way of introducing themselves and sharing who they are, at least as a leader, all of which were specific themes in this study. 
Overview of professional trajectories. Altogether, all of these participants started their careers as a teacher and faculty in higher education between the 1970s and 1980s. Later they moved into some level of management or administration because of one impetus or another, and all have had at least one or more presidencies and/or chancellorships in their careers. At the time of this study, all participants were retired from such leadership positions, but all were also very busy during this phase of their lives. For example, all but one participant, Debbie, are currently involved in teaching and/or consulting with different systems or agencies in higher education.

Overview of no confidence votes of participants. Of the four participants, three (3) (Susan, Paul, and Debbie) received a vote of no confidence on their leadership, a total of four (4) votes, with one participant, Paul, receiving two separate votes at two different institutions. In the case of the fourth participant, Bill, he reported receiving a failed vote. According to Bill, a failed vote means that when the votes were counted, more faculty members voted to reject the vote of no confidence in his leadership. However, the articles about Bill's vote run contrary to his narrative, both in the press and from a state reporting agency, which indicated that he did receive a vote of no confidence. Still, at no time, these reports did not provide actual counts of the votes in these documents and this data was not apprehended by this researcher. 
One of the reasons for the missing data about Bill and the rest of these leaders is partly due to data history and maturation (Fraenkel $\&$ Warren, 2008), and specific details about these votes for each of these leaders were limited to campus meeting minutes and local newspapers. Therefore, the effects of data maturation on the internal validity of this study were controlled and consistent for each of these participants (Creswell, 2007). Nevertheless, the key reason for keeping this participant in the sample is because this misinformation about the vote has continued to follow this leader from the time the vote was given (and failed) to present, allowing Bill to contribute an especially rich narrative to this study. Overall, the participants, their leadership experiences, the rationales for each of these votes were as varied as the situations that led to the vote itself, and each participant's narrative identified key events or interactions in his/her story to demonstrate this point.

Below, Table 6 summarizes the key demographics and background information of each participant.

Table 6

Votes of No Confidence for Each Study Participant

\begin{tabular}{|l|l|l|l|l|l|l|l|}
\hline $\begin{array}{l}\text { District/ } \\
\text { College }\end{array}$ & CEO & $\begin{array}{l}\text { M } \\
\text { I }\end{array}$ & Action/Reason & $\begin{array}{l}\text { Vote } \\
\text { Dates }\end{array}$ & $\begin{array}{l}\text { District } \\
\text { Response }\end{array}$ & $\begin{array}{l}\text { CEO Status } \\
\text { as of 2006 }\end{array}$ & $\begin{array}{l}\text { Researcher's } \\
\text { Notes }\end{array}$ \\
\hline $\begin{array}{l}\text { Sunshine } \\
\text { College }\end{array}$ & Bill & $M$ & $\begin{array}{l}\text { President was } \\
\text { hired as "change } \\
\text { agent" and faculty } \\
\text { thought proposed } \\
\text { changes were too } \\
\text { aggressive; they }\end{array}$ & $\begin{array}{l}\text { Mid } \\
\text { I990s }\end{array}$ & $\begin{array}{l}\text { CEO } \\
\text { resigned: } \\
\text { he and } \\
\text { board } \\
\text { were "not } \\
\text { on the }\end{array}$ & $\begin{array}{l}\text { Became } \\
\text { interim VP } \\
\text { for student } \\
\text { services at } \\
\text { (another } \\
\text { CC); then to }\end{array}$ & $\begin{array}{l}\text { Bill is } \\
\text { currently } \\
\text { retired and } \\
\text { teaching } \\
\text { part-time at } \\
\text { a local }\end{array}$ \\
\hline
\end{tabular}




\begin{tabular}{|c|c|c|c|c|c|c|c|}
\hline & & & $\begin{array}{l}\text { went to media; } \\
\text { not enough } \\
\text { community } \\
\text { support for new } \\
\text { president. }\end{array}$ & & same page" & $\begin{array}{l}\text { state } \\
\text { Chancellor's } \\
\text { Office; hired } \\
\text { as supt/pres } \\
\text { at (another } \\
\text { CC), } 2000 \text {; } \\
\text { left for } \\
\text { private } \\
\text { business in } \\
2002\end{array}$ & university \\
\hline $\begin{array}{l}\text { Queens } \\
\text { College }\end{array}$ & $\begin{array}{l}\text { Debb } \\
\text {-ie }\end{array}$ & $F$ & $\begin{array}{l}\text { Vote taken in } \\
\text { response to } \\
\text { administrative } \\
\text { reorganization } \\
\text { eliminating some } \\
\text { positions; } \\
\text { coincided with } \\
\text { fiscal cutbacks. }\end{array}$ & $\begin{array}{l}\text { Mid } \\
\text { I990s }\end{array}$ & $\begin{array}{l}\text { Strong } \\
\text { board and } \\
\text { district } \\
\text { CEO } \\
\text { support; } \\
\text { improved } \\
\text { communica } \\
\text { tions } \\
\text { (reinstated } \\
\text { staff } \\
\text { newsletter } \\
\text { ) } \\
\text { established } \\
\text { committee }\end{array}$ & $\begin{array}{l}\text { Remained } \\
\text { college } \\
\text { president; } \\
\text { became } \\
\text { chancellor of } \\
\mathrm{KC} \text { College } \\
\text { in } 1999 .\end{array}$ & $\begin{array}{l}\text { Debbie is } \\
\text { currently } \\
\text { retired and } \\
\text { involved in } \\
\text { community } \\
\text { projects }\end{array}$ \\
\hline $\begin{array}{l}\text { Jago } \\
\text { College }\end{array}$ & Paul & M & $\begin{array}{l}\text { Vote taken as part } \\
\text { of contract } \\
\text { negotiations }\end{array}$ & $\begin{array}{l}\text { Mid } \\
1990 \mathrm{~s}\end{array}$ & $\begin{array}{l}\text { Mgt and } \\
\text { the board } \\
\text { took a } \\
\text { pro- } \\
\text { confidence } \\
\text { vote; } \\
\text { resulted in } \\
\text { editorial } \\
\text { accusing } \\
\text { faculty } \\
\text { bargaining } \\
\text { unit of } \\
\text { unfair } \\
\text { labor } \\
\text { practice. }\end{array}$ & $\begin{array}{l}\text { Remained } \\
\text { district } \\
\text { superintende } \\
\text { nt, until } \\
\text { moved to } \\
\text { Campion } \\
\text { College as } \\
\text { CEO in } 2002 \text {. }\end{array}$ & $\begin{array}{l}\text { Paul is } \\
\text { currently } \\
\text { retired and } \\
\text { does } \\
\text { consulting }\end{array}$ \\
\hline $\begin{array}{l}\text { Campion } \\
\text { College }\end{array}$ & $\begin{array}{l}\text { Paul } \\
\text { 2nd } \\
\text { vote }\end{array}$ & $M$ & $\begin{array}{l}\text { Vote by senate } \\
\text { presented "Bill of } \\
\text { Particulars" } \\
\text { protesting layoff } \\
\text { notices and citing } \\
\text { lack of } \\
\text { consultation with } \\
\text { faculty during } \\
\text { collective } \\
\text { bargaining } \\
\text { negotiations. }\end{array}$ & $\begin{array}{l}\text { Early } \\
2000 \text { s }\end{array}$ & $\begin{array}{l}\text { Statement } \\
\text { of support } \\
\text { from } \\
\text { board, } \\
\text { CEO } \\
\text { responded } \\
\text { to Bill of } \\
\text { Particulars. }\end{array}$ & $\begin{array}{l}\text { Remained in } \\
\text { the position } \\
\text { until } 2008 \\
\text { and retired } \\
\text { rev. LT }\end{array}$ & $\begin{array}{l}\text { Paul is } \\
\text { currently } \\
\text { retired and } \\
\text { does } \\
\text { consulting }\end{array}$ \\
\hline
\end{tabular}




\begin{tabular}{|c|c|c|c|c|c|c|c|}
\hline $\begin{array}{l}\text { Hugh } \\
\text { College }\end{array}$ & Susan & $F$ & $\begin{array}{l}\text { Board support for } \\
\text { CEO; Board chair } \\
\text { \& CEO met with } \\
\text { faculty leaders; } \\
\text { implemented } \\
\text { strategies to } \\
\text { increase faculty } \\
\text { involvement }\end{array}$ & $\begin{array}{l}\text { Mid } \\
1990 \mathrm{~s}\end{array}$ & $\begin{array}{l}\text { Remained } \\
\text { district } \\
\text { chancellor. }\end{array}$ & unknown & $\begin{array}{l}\text { Susan is } \\
\text { currently } \\
\text { retired but } \\
\text { recently } \\
\text { served as an } \\
\text { interim } \\
\text { president } \\
\text { and does } \\
\text { some } \\
\text { consulting } \\
\text { part-time }\end{array}$ \\
\hline
\end{tabular}

Note: This table is a summary each participant and a synopsis about when they received the vote and what happened after the vote. It also presents an update concerning what they are doing, currently. Data was adapted from no confidence vote documents found in newspapers and reports and outline in a conversion table in Appendix F, p. 505.

Furthermore, it should be noted that the data from other sources have been placed in a data conversion table to maintain privacy (see Appendix F, p.505).

These documents can include the following:

- Policy documents

- Bill of Particulars (a document prepared and given to the board and leader that outlines the concerns and grievances of the faculty or group giving the vote),

- Newspaper articles

- Reporting agency documents

Each of these documents will be referred to as research, newspaper, agency, or policy documents with a number attached to it throughout the rest of this study. 
Inclusion/Exclusion criteria. This study required that all selected participants met the following criteria: (a) identify as a current or former college or university president; (b) have worked for at least one to five years or more in the same institution in which they received a vote, and at least one to two years in the position of the presidency, and (c) have had at least 8-10 years of cumulative experience in higher education. All participants met this requirement, amassing over 30 years each in higher education, and many of them served as a president or chancellor for at least one year in the institution in which they received the vote.

\section{Data Collection Procedures}

As the sole researcher, I conducted two one-hour in-depth interviews with each participant or key informant, using a semi-structured interview protocol, to explore the crisis under consideration (see Appendix C, p. 496). This protocol was used to gather both the descriptive and essential data for the research focus, as well as to avoid any sort of type III errors as described by Schwartz and Carpenter (1999). These types of errors involve misaligning the research focus with the research questions, or, in other words, by asking the wrong questions throughout the interviews and study (Schwartz \& Carpenter, 1999).

Each one-hour interview was audio-taped and were either conducted inperson (face-to-face) or using secure remote and synchronous technology (e.g., Skype, other videoconferencing alternatives, etc.) already used by the participants. 
All information from these key informants was collected using field or observer notes as well as via a digital audio recording device. As a result, in-person interviews were the primary method of data collection; however, remote and synchronous technology was used with one participant who was inaccessible to the researcher. Participants were repeatedly reminded that this researcher only retained audio recordings using a digital recording device. In addition, all participants were reminded that all identifiable data would be redacted from the study as much as possible and that a data conversion table would be used to protect their privacy as well.

\section{Using Asynchronous Interview Methods}

It is also important to note here that synchronous or real-time interviews have been used in more recent research and in different disciplines such as Skype interviews with military veterans who are amputees in psychology (Cater, 2012), medical and radiation sciences (Bolderston, 2012) and social sciences (Deakin \& Wakefield, 2013). Researchers have noted two major benefits of using a videoconferencing tool. It is cost-effective for qualitative research (Cater, 2012), and it allows researchers to gain access to participants dispersed globally (Bolderston, 2012). Researchers who use such technology should try to closely replicate an in-person interview process as much as possible by using parameters similar to an in-person interview protocol (Cater, 2012). However, there may be risks associated with remote interviews, such as a loss of rapport, visual cues may 
be hidden from the researcher, participants can be distracted, or participants may find it much easier to withdraw from the study or end an interview, compared to an in-person interview (Deakin \& Wakefield, 2013). Such risks can be minimized by briefly meeting with the participants to ensure that they are comfortable with the technology of their choice, requesting that participants provide uninterrupted time and avoid distractions such as checking emails, cell phones, Facebook, etc., and that the researcher keeps a journal of his/her observations similar to those used for an inperson interview (Deakin \& Wakefield, 2013). This study incorporated these parameters and recommendations to minimize potential risks.

\section{Data Triangulation and Member Checking Methods}

As an outsider and a non-participant observer in this study, I also used data memos to record my reflective thoughts, prejudices and biases to help reduce any researcher threats to the internal validity of study (Fraenkel \& Wallen, 2008). In addition, data from each interview were corroborated or triangulated with multiple sources of data, such as newspaper briefs, institutional documents, that were collected and then crosschecked with the stories that these leaders construct (Fraenkel \& Wallen, 2008). Other additional sources used for triangulating these stories included senate meeting minutes and all other types of documentations available or provided to the researcher. 
A key component of the triangulation process was to include a strategic plan for reliability through a member checking process, as described by Hochschild (2009), when interviewing elite participants, such as community college presidents and executives. The member checking was used and conducted during interviews with each leader, where they were asked to respond to specific information found in NCV newspapers, journals or meeting minutes during their actual interviews, instead of asking them to verify this information after the face-to-face interviews. This strategy not only helped in the member checking process but was useful in the triangulation process to help with consistency, clarify timelines, and to understand the different perceptions in these stories (Bogdan \& Biklen (2007). No secondary member checking process was completed, to avoid additional threats to this process, such as participants changing their minds or reframing their words in light of how they might appear or ultimately, withdrawing their participation. Therefore, member-checking was completed during these interviews.

\section{Data Analysis Procedures}

Using an interpretive approach, this study incorporated a thematic analysis of all the data collected to increase the probability of capturing the subjective meanings of each participant. Once the data were prepared for analysis by creating line-by-line (verbatim) transcriptions of each interview, this information underwent several reviews by the researcher, in an effort to analyze each transcript and note 
emerging patterns and clusters found in the data, using Bogdan and Biklen's (2007) six steps for thematic analysis, combined with Creswell and Plano Clark's (2011) qualitative research design. Those steps will be detailed below. This approach was chosen procedures because it was the designed that this researcher was most familiar with and had used in a previous pilot study with other executives. In addition, this study also considered Moustakas's (1994) interpretive and heuristic phenomenological approach, primarily because he is considered the father of phenomenology. The focus of this research paradigm is on the researcher and the researched. Meaning, it is focused on the researcher being able to put his or her own biases and assumptions aside, as well as it is focused on the participant's selfawareness and their discovery and description of self in a situation. However, a major criticism of this research approach is that it failed to delve into the ways the external environment or context can influence the meanings individuals may give about their situations, which this study finds critical (Applebaum, 2011). This study will focus on both these presidents' sense of self and their experiences within these community college contexts to better understand their stories about the attributable factors and consequences that may have led to these NCVs.

Overall, a major strength of this specific approach is that it can capture the experiences of those who have experienced a phenomenon, which has been difficult to study. A weakness to this approach is that it is not generalizable to all individuals who may experience a NCV phenomenon, and some of the knowledge 
may or may not have transferrable data to similar situations. However, knowledge about the phenomenon is being produced. Thus, this researcher felt comfortable using Weick's (1995) sensemaking approach alongside the methodology described in Bogdan and Biklen's (2007) and Creswell and Plano Clark's (2011) to complete the data analysis process.

\section{Procedures Overview}

Once all iterations of reviewing the transcripts were complete, all the emerging patterns and clusters were indexed into codes, and then these codes were placed into themes and sub-themes, using the research questions as a guide. All themes and their sub-themes were organized according to the themes found in literature, such as Karl Weick's (1995) seven properties for understanding the sensemaking or meanings these leaders give to these crisis situations. This study also incorporated technologies, such as NVIVO 10 software, to analyze the themes found in each transcript, allowing the researcher to cross-check codes and themes between the manual and electronic processes. All themes were then compared and calibrated to ensure the highest levels of accuracy in the data. The analysis also included data auditing and member-checks to review the transcripts and themes, determining any threats to the credibility and reliability in the data. 


\section{Data Analysis through Stories}

A major premise used in the data analysis process was that there are at least two sides to every story. With the limited to non-existent voices of these CEOs and their stories in the literature, the structure of the interview process was the designated space given to these leaders to tell their stories. For example, for each one-hour session with the leader, the interviews were divided to help each leader share their thoughts, sequentially. Meaning, in the first interview, the focus was on these leaders' lives, identities, philosophies, professional trajectories, leadership practice, and the situations that they believed led up to the vote. This was done to build rapport with each participant and to encourage them to share as much information about themselves as possible.

By the second interview, usually conducted between days and weeks after the first, the focus was on what happened when they heard they got the vote, the aftermath of the vote, the lessons they learned. Therefore, focusing on the stories or constructed narratives of these leaders offer a way of affording these executives a space for sharing their sensemaking of what happened to them during these votes.

However, by carving out this space, it was also clear that extra time was needed for each participant to tell their stories, and this researcher found it difficult to interrupt these elite executives while they shared their experiences. As a result, for all participants, except one, each planned one-hour interview was extended by an additional 15 to 30 minutes for most participants. 
Hochschild (2009) described the storytelling process as a means for obtaining as close as possible a "complete, honest, as nuanced a story as possible" particularly from elite participants during intensive interviews (para. 4). Hochschild (2009) explained that the term elite does not refer to "high social, economic or political standings" of individuals, but it refers to the name and type of positions these individuals are in (para. 1). She further explained that the term intensive interviews refers as interviews lasting "one hour or more" with individuals in specific types of positions as opposed to some random group of individuals (para. 1). She suggested that storytelling allows the researcher to "probe more deeply" into the thoughts and rationality of these participants with the hope to get past the superficial often scripted and expected responses (Hochschild, 2009, para. 6). However, Hochschild (2009) also admonished her fellow researchers to avoid wasting the time of participants by ensuring that they know the topic and context well, and to also recall what these participants said or did in previous years in an effort to "portray interviews fairly... [by giving] the reader much evidence to show the complexities of the problems... without distorting historical record as revealed by respondents (para. $10 \& 11$ ). Hochschild (2009) also asserted that researchers must be rigorous in analyzing such interviews by being aware of the potential "noise" in these stories, which could include the "hesitancies...disconnections, anomalies...apparent inconsistencies... [and] even incoherence" and by paying attention when reviewing the transcribed texts (para. 8 and 9). A final advice to the 
researcher was to also avoid the "temptation to ask them one's own research questions" but to leave some space between onself and the respondents (para. 12). This final advice was particularly helpful to this researcher who was often tempted to throw such cautions to the wind during these interviews. Overall, discussed the rigor needed to understand and capture the fundamental narrative with substantive context for one's research.

Weick (2001) also described storytelling as an artful and powerful practice and tradition still followed around the world, and used as a means for learning about myths, traditions, and realities. Such stories are like painting a picture: a way of constructing and sharing our own reality (Barnes, 1984). Other researchers agree that stories or narratives act as a powerful mechanism that allows the storyteller to explain and diagnose the situations they have experienced (Basbøll, 2012; Fraenkel \& Wallen, 2008), retrospectively recalling what they saw, knew and did (Weick, 2001). Likewise, stories offer a means for the storyteller to reflect and reorganize his or her thoughts into sometimes plausible and logical ways of explaining an event or experience in his/her life (Latour, 2011; Weick, 2001), which may not necessarily be accurate (Basbøll 2012), but can still be used as a reasonable guide or map for the future (Weick, 2001).

It is also important to note that with this story based format most leader participants were often open to discussing most topics, but closed to others, as Hochschild (2009) advised. These leaders were also able to tell their stories best 
during open-ended and semi-structured questions. However, sometimes these participants were closed to specific details such as whether or not they received a contract payout and how much they received, or whether or not they may have been asked to resign quietly, etc. In some instance, these leaders asserted that they were unable to share certain details because of previous legal agreements with these institutions or they did not recall the intricate details of certain events.

Delgado (1989) further explained that it is not enough to simply tell or get stories from individuals, but it is critical to understand such stories within their context as counter stories or narratives. He explained that these are the stories or "counter-narratives [that] members of an outgroup [outsiders in their own group]...tell themselves and others... [about their] 'counter-reality'... [or a] plausible 'counter-view' of what happened", especially during a crisis event (pp. 2434-2435). Thus, considering the narratives of these CEO participants as counternarratives is a key element in this research design. Stanley (2007) also used the concept of counter-narratives in her description of the problems African-American faculty and researchers were experiencing when they tried to publish their research and teaching experiences in main-stream and traditional journals. She is suggesting that the work or research of these African- American scholars were counternarratives and that these scholars' work were often precluded because the discourse used were often different from the mainstream discourse and controversial for some journal reviewers. Stanely (2007) found that African-American faculty and 
researchers eventually found and utilized nontraditional platforms as an outlet to share their work and their voices.

Weick (2001) suggested that a crisis event acts as an occasion for individuals to try and make sense of what is going on within their institutional contexts (Maitlis \& Sonenshein, 2010; Weick, 2001). As a result, many counternarratives can emerge out of such contexts as individuals reflect on their experiences and try to make sense of it. Therefore, as faculty or faculty unions present their narratives in these mainstream reports about why they voted against the leadership, the leader's counter-narratives will be examined alongside the narratives of these institutional players, whose accounts of the crisis is often well anchored in the media.

Through these rich and polythetic counter-narratives of these presidents, this study was able to analyze what may have been going on before, during, and after such a crisis, from the perspective of the president. As a strategy for increasing the strength of validity or plausibility and credibility in this study, these counternarratives were be triangulated with the narratives and sensemaking presented in other documents, such as newspaper articles or institutional documents to see what they all have to said about such events, a design supported by other researchers (Bogdan \& Biklen, 2007; Creswell, 2007; Fraenkel \&Warren, 2008). 


\section{Data Analysis: A Six-step Process}

As previously noted, the systematic process for analyzing the datasets collected was taken from the work of both Bogdan and Biklen (2007) and Creswell and Plano Clark (2011) and modified into a six-step process for this study's purpose. The study's data analysis process analysis included personally transcribing all in-depth interviews of each participant. Several iterations of reviewing the transcripts were completed before moving on to the next step of data analysis. During these reviews, other documents were examined to see if there were any links, connections, or disconnects among these narratives and those of institutional players or documents concerning no confidence votes (NCVs). For this study, those documents included all of the data memos, newspaper articles, policy documents, and reports, along with the audio-tapes and the in-depth interview transcripts.

The next step of data analysis process was inductive. This analysis searched for any emergent and substantive themes in the data about the phenomenon. This analytical process is a "process of systematically searching and arranging...data... into manageable units, [by] coding them, synthesizing them and searching for patterns" (Bogdan \& Biklen, 2007, p. 159). To do this, this research looked for emergent thoughts and perspectives from each participant. These thoughts were then coded, a systematic way "of grouping evidence and labeling ideas... to reflect ...broader perspectives" (Creswell \& Plano Clark, 2011, p. 208). Creswell and Plano Clark (2011) outlined a process for getting to know the data, selecting and 
labeling text, looking for "inter-coder agreements," combining themes, and using "cross-case thematic analysis" (p. 309). Table 7 provides an overview of the step outlined by these scholars and Table 8 provides an outline on how this study used each step below.

Table 7

Methods: Bogdan and Biklen's (2007) Combined with Creswell and Plano Clark's (2011) Research Design

\begin{tabular}{|c|c|}
\hline Steps & Process \\
\hline 1 & Read through the transcripts carefully \\
\hline 2 & $\begin{array}{l}\text { Code data by segments or index and label } \\
\text { the text (used NVIVO } 10 \text { software) }\end{array}$ \\
\hline 3 & $\begin{array}{l}\text { Review and verify codes in software as } \\
\text { many times as needed until emergent } \\
\text { themes are formed }\end{array}$ \\
\hline 4 & Use codes to aggregate themes \\
\hline 5 & Connect and interrelate themes \\
\hline 6 & Check for inter-coder reliability \\
\hline
\end{tabular}

Note. Adapted from Bogdan and Biklen's (2007) and Creswell and Plano Clark's research methods process for data analysis.

Table 8

Six Steps for Coding and Thematic Analysis Process 


\begin{tabular}{|c|c|c|}
\hline Step I: & $\begin{array}{l}\text { Read and re- } \\
\text { read data }\end{array}$ & $\begin{array}{l}\text { Completed several cycles of reading and re-reading interview } \\
\text { transcripts and other documents to explore links across all data and } \\
\text { between the interview data and research questions (Creswell et al., } \\
20 \mathrm{II} \text { ). }\end{array}$ \\
\hline Step 2: & $\begin{array}{l}\text { First Cycle } \\
\text { Coding: Code } \\
\text { units of data }\end{array}$ & $\begin{array}{l}\text { Using NVIVO IO Software- all transcripts, memos and other } \\
\text { documents were uploaded as case nodes or buckets for each } \\
\text { participant. From these case nodes, the first cycle of coding began. } \\
\text { This type of coding involved identifying words, sentences, paragraphs } \\
\text { of data in each interview or document and then indexing or labeling } \\
\text { this information (Bogdan \& Biklen, 2007). This process rendered an } \\
\text { extensive list of codes for each participant. }\end{array}$ \\
\hline Step 3: & $\begin{array}{l}\text { Second Cycle } \\
\text { Coding-Develop } \\
\text { categories of } \\
\text { data }\end{array}$ & $\begin{array}{l}\text { Using NVIVO Software, this process involved sorting and resorting } \\
\text { data for each participant, within each case node for each participant, } \\
\text { and then creating a family of categories (hierarchies) or labels to see } \\
\text { where the codes and themes overlapped, converged or diverged } \\
\text { (Bogdan \& Biklen, 2007; Creswell et al., 2011). }\end{array}$ \\
\hline Step 4: & $\begin{array}{l}\text { Third Cycle } \\
\text { Coding- Coding } \\
\text { by answering } \\
\text { the research } \\
\text { questions } \\
\text { (structural } \\
\text { codes) }\end{array}$ & $\begin{array}{l}\text { Using NVIVO Software, similar to steps I and } 2 \text {, this process allowed } \\
\text { the researcher to review the interviews with fresh eyes and compare } \\
\text { and contrast previous codes. All transcripts were re-coded and } \\
\text { placed into new categories or themes based on the interview } \\
\text { protocol questions, i.e., background information, culture and climate, } \\
\text { etc. This information was stored under each case node as a separate } \\
\text { node for each participant. These codes were then used as an inter- } \\
\text { rater codes, as if a second person were analyzing the data (Creswell } \\
\text { et al., } 20 \mathrm{II} \text { ). }\end{array}$ \\
\hline Step 5: & $\begin{array}{l}\text { Synthesizing } \\
\text { themes } \\
\text { (frequency of } \\
\text { codes) }\end{array}$ & $\begin{array}{l}\text { Using NVIVO Software, the codes from cycles } 2 \text { and } 3 \text { were placed } \\
\text { into queries for places were the data overlapped or were divergent } \\
\text { to better understand the story in the data. This process included } \\
\text { both auto coding and matrix coding schemes, using the software, } \\
\text { which will be discussed below. }\end{array}$ \\
\hline
\end{tabular}




\begin{tabular}{|l|l|l|}
\hline \multirow{3}{*}{ Step 6: } & $\begin{array}{l}\text { Grouping and } \\
\text { choosing } \\
\text { themes from } \\
\text { steps 2-5 to see } \\
\text { what the data is } \\
\text { saying }\end{array}$ & $\begin{array}{l}\text { Using NVIVO Software, each major category or significant theme } \\
\text { from steps 2-5 was analyzed and weighted against, both (a) } \\
\text { references-how much of the data was covered in that theme, and } \\
\text { (b) matrix coding-using the artificial intelligence portion of the } \\
\text { software to determine where thematic overlaps were present in the } \\
\text { overall coding process. The most significant and salient themes were } \\
\text { then chosen for this process. }\end{array}$ \\
\hline
\end{tabular}

Note. An NVIVO 10 software package was used to store, manage, and interrogate the data to see determine structural themes, frequencies of themes and most salient themes in the study.

As noted in Table 8, each coding and thematic cycle involved finding the most salient concepts used by the participants when they outlined their stories or counter-narratives. There were several iterations of coding and recoding potential themes; starting with creating a large index of specific words of these leaders; then using the actual interview protocol questions, and then triangulating data memos, newspaper reports and other documents to find all emergent codes.

These codes were then placed into categories or aggregate codes, such as codes described in Bogdan and Biklen's (2007) work. For example, these scholars suggested ways to categorize and organize data and was such categories were used in this study, to include: the leader's "institutional" and "social context" in the organization, "relationships" with constituents, the "perspectives" and "thinking" about leadership and the vote, "strategies" employed by participants to get through the vote, and the lessons they learned and shared in their narratives (Bogdan \& 
Biklen 2007, p. 172-180). These types of aggregated codes created nodes or bins to filter in codes that could belong to one or more family of codes, and an excellent way to organize and re-organize all codes during several iterations of coding. Another key element of this data analysis process was ensuring that codes and themes, also known as nodes or bins for storing codes, were based on the direct or exact words of these participants, also known as "in-vivo" coding (Creswell 2007, p. 153). All data analysis was prepared in the NVIVO 10 software and reported in Excel documents for further assessment.

Once these themes were sorted as well as aggregated, they were also prepared for an auto-coding cycle using the NVIVO 10 software's artificial intelligence software. This auto-coding process was used to determine points of agreement and disagreement between the codes manually created by the researcher and that of the system, a form of matrix coding, to "compare patterns" and check for reliability in the analytical process (NVIVO, 2015). To measure this consistency or reliability in the coding process, NVIVO 10 used Cohen's Kappa Coefficients, which are statistical quantities or measurements that "take into account the amount of agreement that could be expected to occur through chance" (NVIVO, 2015, para. 3). In addition, the codes derived from each cycle of existing codes created manually, including codes from the protocol questions, were all tested against the codes suggested by the system. Essentially, the artificial intelligence used these existing codes to test its own patterns of codes when it searches through 
the entire transcripts, sentences and paragraphs. Then it creates its own set of codes as options for this project. Because this particularly functionality of the software is still in its beta stage, it was more useful in findings areas in the text that may not have been coded at all for the researcher to review again. Its most impactful usage was gained with its inter-rater codes that were presented in the form of a Kappa Coefficient. The findings below in Table 9 demonstrate these findings with comparisons of manual and auto-coding data.

Table 9

Comparisons between the Researcher and NVIVO 10's Artificial Intelligence Themes

\begin{tabular}{|c|c|c|c|}
\hline Participant's Interview & Kappa & Agreement (\%) & Disagreement (\%) \\
\hline Debbie & 0.9069 & 97.32 & 2.68 \\
\hline Bill & 0.9444 & 97.55 & 2.45 \\
\hline Paul I & 0.9657 & 98.48 & 1.52 \\
\hline Susan & 0.9669 & 98.49 & 1.51 \\
\hline Kappa value & A Kappa value was created by comparing the codes of User A: \\
& Researcher LT and User B: NVIVO Artificial Intelligence that \\
& acts like a second coder. The below key is a guide for \\
understanding these comparisons. \\
One approximate set of guidelines for interpreting the value of Kappa is: \\
Kappa values Interpretation \\
Below 0.40 Poor agreement \\
$\begin{array}{l}\text { 0.40 - } 0.75 \\
\text { Over } 0.75 \\
\text { Fair to good agreement } \\
\text { Excellent } \\
\text { agreement }\end{array}$ \\
\hline
\end{tabular}

Note. The data represent the rate of agreement or disagreement between the manual coding processes of the researcher vs the artificial intelligence coding process of the NVIVO 10 
software. This data illustrates that very little disagreements were found in the final manual codes and the artificial codes produced by NVIVO.

\section{Significance of the Study}

The significance of this research is first to contribute to the dearth of empirical literature on this particular phenomenon, by giving voice to the leaders who experience these leadership crises, and finding possible factors that can contribute to these crises. Furthermore, this issue is of even greater concern as institutions nationwide prepare for the imminent crisis of a leadership gap in the college presidency, slated to be the result of the pending leadership retirement and the lack of individuals in the traditional pipelines (American Council on Education, 2007). This problem of filling the leadership gap compounds the challenges of attracting and retaining leaders for these positions (ACE, 2007). Most importantly, this study is a potential knowledge fund for future aspiring leaders.

Another major significance of this study is to increase awareness about the barriers or mishaps leaders can face in their organizations. Several recommendations for averting and possibly overcoming such barriers are presented in this study, with the intention of increasing awareness about the issues or critical events that can limit or reduce the leader's longevity and leadership agendas.

\section{Internal and External Validity}

Although the findings in this study may not be transferrable to other organizations or to every leader, those findings will provide plausible stories of how 
leaders experience a phenomenon that very few leaders experience and many others never expect. Therefore, by describing the contexts, assumptions and sensemaking of these leaders, other aspiring or current leaders can better understand this phenomenon and will be able to transfer the knowledge applicable to their situations.

Similarly, this research proposes to carefully examine and present a faithful narrative of these leaders, as they agreed to share their stories of this critical event. In addition, participants may be asked to engage in member-checking and feedback to ensure that the data used are credible and reflect their stories accurately, and that these stories are free of the researcher's own judgments and biases (Bogdan and Biklen, 2007). All these steps were taken to increase and maintain both internal and external validity within the study.

\section{Creditability}

Creswell (2007) using Lincoln and Guba's (1985) work on data justification and data reliability and validity, asserted that this criterion focuses on the getting as close to accurate stories from participants as possible. Therefore, all interviews were conducted using the same manner and format with two interviews per participant, and the same planned duration for each. Each transcript was transcribed verbatim as a means to stay close to the narrative. As noted earlier, member-checks occurred during each interview via follow-up questions and clarifying questions posed to each participant. Each story was confirmed by 
triangulating sources and by asking follow-up questions during each interview. Data auditing was also completed by me, the researcher and by chair, who briefly and preliminary audited codes and themes. Data memos- were also used to help me, the researcher, place some distance between myself and the process as much as possible. All of which were used to limit or reduce any threats to internal validity as much as possible

\section{Dependability}

Dependability focuses on the reliability or repeatability of the research process (Creswell, 2007). For this reason, each interview used the same interview protocol and format for each participant only deviating to ask questions specific to the respondent's situation or in support of the triangulation process. As a result, other researchers interested in this study should find the protocol useful.

\section{Plausibility}

Plausibility refers to the quality, rigor and trustworthiness of the data and findings, and the term is being used here although Creswell (2007) doesn't actually use the term. As previously noted, this study used audio-tapes to capture the stories of each participant, which were then transcribed verbatim by me, the researcher, and evaluated using in-vivo codes and themes as described by Creswell and Plano Clark (2011), which are based on the words of participants. A list of these codes and themes were tested against the artificial intelligence of the NVIVO 10 software 
to determine to identify similar and different coding patterns. In addition, Kappa Coefficients were used to verify the reliability of the manual codes when compared to the NVIVO's codes.

\section{Ethical Considerations}

This study utilized the highest sense of confidentiality and the protocol, as prescribed by the San Francisco State University's Institutional Review Board (IRB), for the protection for human subjects. All four participants were required to and did complete a consent form, acknowledging the confidentiality, privacy and care that will be used to protect their data, and allowing them to withdraw from the study at any time (see Appendix B, p. 493). None of the participants' names, institutions or identifiable characteristics was used in this study.

In addition, participation in the study involved minimal risk. One potential risk is loss of privacy. This risk was minimized by keeping all research data in my home office lockbox, using password-protected files accessible only to the researcher as described by Bogdan and Biklen (2007). All electronic data were kept in an encrypted document on a password-protected computer. Since prospective participants may have continued to be leaders in the field, all recruitment scripts, consent forms, and communications, both in-person or via email, emphasized the voluntary nature of the participation. Moreover, refusing to participate had no consequences whatsoever on the participant who did decline to be interviewed. 
Another potential risk is the slight chance that campus insiders could infer the identity of participants since the situations surrounding each of these votes have been and will be made available to the public. All names or identification information of individual participants were changed or omitted in the study, and all data in the finished report was presented in an aggregated form. In addition, extra steps were taken to maintain the privacy and identifiability of participants through the use of Document Conversion table (see Appendix F, p. 505). This is a table that simply outlined the type of document found about the participant, and included a brief description of the article with the date of the document, for example. These documents included newspaper and journal articles, with direct reference to each participant and the events surrounding his/her vote. This strategy was used a solution to take due care in maintaining the privacy and protecting the participant as much as possible.

\section{Role of the Researcher}

In this study, my role was to collect, analyze, and interpret data in this study; therefore, I am the primary and sole instrument for collecting and analyzing the data. As the main data instrument, I conducted all the interviews, writing all my reflexive data memos, and working with data auditors and key informants to ensure validity and reliability in all areas of the study. Because I have never worked in any of the California community college campuses in the past, I did not know nor have 
any previous relationships with any of the key participants, and I worked quickly to build rapport with each of the leaders because of the limited time during each of the two interviews. 


\section{Chapter Four}

\section{Findings and Results}

The purpose of this phenomenological study was to explore how CEOs in California Community Colleges made sense of no confidence votes (NCVs) on their leadership. The study's specific aim was to find out the implications of no confidence votes on presidents by examining the factors that CEO participants identified and attributed to these events. The findings from this study were drawn from the stories of each participant and what they identified as the factors contributing to the vote, and the lessons they learned, if any. This chapter is organized into three major sections (a) overview of the data analysis strategy and process; (b) demographic and background information of each participant, including information about the vote for each participant, and (b) research findings. The chapter will then close with a summary of these themes and conclusion.

\section{Data Analysis and Instrumentation}

Through these presidents' lenses, the inductive process to answer this study's primary research questions (Creswell, 2007). The data analysis preliminary phase began with ensuring that the research instrument (interview protocol) and the research questions were aligned with the purpose of this study. This is an important data analysis step because it can help the researcher to avoid what Schwartz and Carpenter (1999) called a Type III error. Type III errors ask the wrong questions 
throughout the interviews and data analysis. Fraenkel and Warren (2008) also suggested that the process of interrogating the research questions is a key process for validating and securing the information needed to understand and interpret data. Thus, to avoid such errors, the study's research questions emerged and were formulated using the limited empirical work found, review of the literature, and theoretical framework, a process supported by other researchers and noted in Chapter Three (Aslam \& Emmanuel, 2010; Bogdan \& Biklen, 2007). These questions were later compared to the interview protocol to check for alignment, and adjusted accordingly. In addition to the research questions, this study also tested and vetted the protocol instrument in two ways: (a) using a previous in-class pilot study with a vice president who experienced a similar incident, and (b) going through an approval process with dissertation chair, a process supported in the literature (Bogdan \& Biklen, 2007). The following table illustrates this process and shows how these questions overlap for this study.

Table 10.

Intersections between Research Questions and Interview Protocol Questions

\section{Research Questions Interview Protocol Questions Categories (IP)}

IP 1, 2, 3: Introduction and background of the leader,

1. How do presidents make sense of a vote of no confidence vote on their leadership? \& description of their leadership approach, style and practice in the organization IP 4: Culture and climate of organization and relationship with faculty

IP 5: The vote and what happened 


\begin{tabular}{|l|l|}
\hline $\begin{array}{l}\text { 2. What factors do } \\
\text { they attribute to the } \\
\text { vote? }\end{array}$ & $\begin{array}{l}\text { IP 6, 7 and 8: The attributable factors that led the } \\
\text { vote, and who led this process }\end{array}$ \\
\hline $\begin{array}{l}\text { 3. What lessons did } \\
\text { these leaders learn as }\end{array}$ & $\begin{array}{l}\text { IP 9, 10 \& 11: Strategies used to resolve the issues and } \\
\text { communicate with constituents to survive this process }\end{array}$ \\
\cline { 2 - 2 } $\begin{array}{l}\text { a result of this } \\
\text { experience, if any? }\end{array}$ & $\begin{array}{l}\text { IP 12, 13, 14: The Aftermath- What happened after the } \\
\text { vote and what lessons were learned }\end{array}$ \\
\hline
\end{tabular}

Note. This is a comparative analysis table of the research and interview protocol questions, used to determine how these questions intersected and supported the purpose of this study. See Appendix C for the full list of protocol questions for this siudy.

In summary, this study's first question was used as the primary and overarching research question and driving force for these findings. The other two questions were also crucial for gleaning the important details about the attributable factors that leaders might identify as contributing to the vote, as well as the key lessons learned from these events, if any. It is important to note that this study's epochè, or insistence on not judging the vote (Creswell, 2007) has always been to draw attention to the narratives of these leaders, as opposed to evaluating the validity or rightness of the vote on their leadership. This study will leave all judgments and conclusions about these votes up to the leader who received them and the readers of this study.

\section{Data Analysis and Themes}

The following tables provide an overview of the datasets used in this thematic analysis and the emergent themes found in the data: 
Table 11.

Overview of Dataset

\begin{tabular}{|c|c|c|c|c|}
\hline $\begin{array}{c}\text { CEO } \\
\text { Participants } \\
\text { (pseudonyms) }\end{array}$ & $\begin{array}{c}\text { Interview } \\
\text { (Pages for both } \\
\text { interviews) }\end{array}$ & $\begin{array}{c}\text { Words in } \\
\text { Interview } \\
\text { document }\end{array}$ & $\begin{array}{c}\text { Interview Codes } \\
\text { (count) }\end{array}$ & $\begin{array}{c}\text { Data } \\
\text { Memos } \\
\text { (count) }\end{array}$ \\
\hline Bill & 148 & 35,330 & 352 & 2 \\
\hline Debbie & 88 & 29,968 & 216 & 1 \\
\hline Paul & 96 & 28,383 & 260 & 2 \\
\hline Susan & 94 & 24,433 & 267 & 2 \\
\hline Total & 426 pgs & 118,114 wds & 1,105 codes & 7 \\
\hline
\end{tabular}

Note. This table illustrates the amount of data obtained and used for the descriptive analysis of CEOs' narratives about these votes.

Table 12.

Significant Cross-Cutting Themes across Study Participants

\begin{tabular}{|c|l|c|}
\hline $\begin{array}{c}\text { Particip } \\
\text { ants }\end{array}$ & \multicolumn{1}{|c|}{ Themes } & \multicolumn{1}{c|}{$\begin{array}{c}\text { References } \\
(\%)\end{array}$} \\
\hline \multirow{4}{*}{ Bill } & Board Relationships (Codes) & 11 \\
\cline { 2 - 3 } & Constituency Relationships (General) & 13 \\
\cline { 2 - 3 } & Family Relationships & 15 \\
\cline { 2 - 3 } & Family (Balancing life and career) & 9 \\
\cline { 2 - 3 } & Shared Governance (Social Structure Codes \& Relationships) & 16 \\
\hline \multirow{5}{*}{ Debbie } & Career Trajectory (General Pathway) & 17 \\
\cline { 2 - 3 } & Faculty Relationships & 10 \\
\cline { 2 - 3 } & Identity (Parental Influences positive and negative) & 9 \\
\cline { 2 - 3 } & Leadership (Style using Strong Team Collaboration) & 11 \\
\cline { 2 - 3 } & Lessons Learned (Plausible Cues and Stories Leaders Share) & 15 \\
\cline { 2 - 3 } & No Confidence Vote (Aftermath: Next steps) & 13 \\
\cline { 2 - 3 } & $\begin{array}{l}\text { No Confidence Vote (Attributable factor Shared Governance } \\
\text { Policies) }\end{array}$ & 10 \\
\cline { 2 - 3 } & $\begin{array}{l}\text { Shared Governance (Leaders practice around shared } \\
\text { governance) }\end{array}$ & 14 \\
\hline
\end{tabular}




\begin{tabular}{|c|c|c|}
\hline \multirow{6}{*}{$\begin{array}{c}\text { Paul } \\
\text { Paul } \\
\text { (cont'd) }\end{array}$} & Board Relationships & 17 \\
\hline & Career Trajectory (General Pathway) & 17 \\
\hline & Family Relationships & 9 \\
\hline & Identity (Construction and Understanding) & 20 \\
\hline & Lessons Learned (Plausible Cues and Stories Leaders Share) & 12 \\
\hline & No Confidence Vote (Rationale for the vote) & 10 \\
\hline \multirow{6}{*}{ Susan } & Career Trajectory (General Pathways) & 16 \\
\hline & Extracted Cues (Problems within the institution) & 10 \\
\hline & Faculty Relationships & 12 \\
\hline & Identity (Construction and Understanding) & 11 \\
\hline & Leadership (Style and Philosophy)) & 9 \\
\hline & $\begin{array}{l}\text { Shared Governance (Leaders practice around shared } \\
\text { governance) }\end{array}$ & 9 \\
\hline Key & \multicolumn{2}{|c|}{$\begin{array}{l}\text { All themes and codes were ranked between } 1 \text { and } 20 \text {, with } 1 \text { being a } \\
\text { less significant code or theme and } 20 \text { being the most significant ones. } \\
\text { Color scales have been applied to locate this information. The above } \\
\text { table pinpoints the areas where each participant thought that } \\
\text { information was significant and important to recall. }\end{array}$} \\
\hline
\end{tabular}

Note: This table highlights the most significant themes as noted in the stories of each participant. The NVIVO 10 Software was very useful in calculating these references or the amount of information coded in each interview, also known as a reference, which rendered a scheme between 1 and 20 of the most reoccurring themes. The table incorporated a temperature scale of colors, with green indicating the most relevant themes in the interview, orange scale indicating the more moderate themes, and pink color scheme illustrating the least relevant themes found in the literature.

Table 12 also acted as a primer for choosing certain codes for analysis and interpretation. For instance, once data were coded in NVIVO 10 Software, each coded dataset was given a percentage called a reference, which highlighted how much of the data had been coded for that code or theme (NVIVO 10, 2015). When much of the data was coded in a second cycle, the more emergent themes emerged particularly when two or more participants spoke about that particular concept. This 
study only focused on major themes, which were categorized and ordered by relationships within themes or nodes (containers for several codes and themes), if any.

For example, the theme career pathway is one category that acted as the parent theme for other categories such as previous leadership positions, or different opportunities. Thus, these categories may have encompassed a small or large portion of each interview, and this category coverage is known as references. Meaning, each category or code $\leq 1$, were considered insignificant because it was only referenced or coverage minimally in the text, and references $\geq 20$, were the highest references so the most significant categories used in this scheme. However, if individual participants had larger references in one particularly areas unique to only that individual, then this particular theme was not included in this scale. For example, Paul, an African American, discussed concerns about being perceived as being arrogant or being told he got the vote from a fellow African American. $\mathrm{He}$ discussed this theme at length with a high reference of 16 ; however, this theme was unique only Paul. For Paul, perhaps, this may have been a form of racially coded communication, a type of dog whistling communication, wherein only certain individual can understand its meanings (Albertson, 2006). As a result, he was able to speak about this issue at length. Similarly, other women participants may have discussed specific incidents that were unique to them and their genders, but were not included in this scale. 
A weakness of the NVIVO 10 software is that it clustered all categories or codes into one major bucket for each person, known as a case node, and such case nodes then aggregated all other case nodes beneath it with large references or coverage percentages. Meaning, once these case nodes were aggregated, some reference points were not included in the table above because these themes were acting like place holders or super parent node with multiple layers of nodes or subthemes collected under a single thematic category or bucket, rather under a single themes. For example, the node Bill had over 760 references, this then become a super parent node or bucket, because the name Bill was the case node or container for all other nodes or codes. Then each theme within this Bill's bucket was further aggregated with references ranging from 199 to 760 . As a result, these large buckets were not included in this research process because these categories acted more placeholders than actual codes.

Essentially, Table 12 also illustrates the key themes that began to emerge, and the places were these themes either converged as well as divergence among the stories of each participant. These themes were then ranked and sorted for each participant's story. The table also illustrated how presidents were trying to make sense of this crisis on their leadership. Some specific areas in which leaders tended to focus were on leadership or leadership styles and practice, career pathways into the presidency, institutional contexts that supported his or her leadership, etc. 
In summary, the results of the data analysis rendered a total of four (4) major themes with 28 subthemes to understand what CEO participants remembered and identified as factors contributing to their votes, and the meanings they believed these votes can have on their leadership. Each of these leaders provided an in-depth interview about no confidence votes on his or her leadership and the situations and circumstances that led to the vote. There were moments when the researcher's expectations about what would be found were not met, such as the leader not taking time to look back to see what the vote meant. However, such unexpected and emergent findings are arguably a normal part of data analysis, and perhaps, a key finding in this study as well (Creswell \& Plano Clark, 2011). The next section of this chapter will focus on the demographic information about these leaders followed by a breakdown of each theme and sub-theme found in the data.

\section{Demographical and Background Information}

\section{Participant: Paul (pseudonym)}

Professional and personal background. Paul identified himself as an African-American male, a native in the state in which he would later have three presidencies and one chancellorship, and as a current retiree with his wife. Paul, now a Chancellor Emeritus and consultant, mentions the importance of his family and is quick to note that his wife was also a college president and that they both had 
very demanding lives and schedules with four, now grown, children between the two of them and several grandchildren. Although proud of both his own accomplishments as well as his wife's. Paul also noted that, at one time in this system, he and his wife were both serving in presidential positions but in different cities, and were trying to raise their family at the same time, and that this type of arrangement soon took its toll on them.

Paul: My wife's name is (Rachel) she is a former educator as well. Matter of fact, at one time in (the state), we were the only two married couples who were presidents.

Paul: No, no, there's been a lot whose husband was and then the wife became [presidents] or the wife was and the husband became after the wife retired. But at one time, we were the only couple who were both presidents at a community college.

...It was hard, you know, because we lived apart a lot because of our careers. Um, when I was at (Campion College), I lived down there in an apartment or condo for six years and only came home on weekends. You know, and when she took the (Rica) job, she was down there a year, I think, or a year and half or two. And then we finally rented out our house and I moved down there and so now we are----we are now back here, in our home here in (Falmouth)... 
... And so I decided and said, "You know, if I really want to make this marriage work and if I am gonna do my part to make this marriage work, we need to live in the same house". And so I applied for a job at (Wrights College) in (a city close near Falmouth)... Then my commute disappeared.

Paul further traced his personal development and leadership style, courage, ethics, discipline, perseverance, and practice, primarily to the ways in which he was brought up and the experiences he had throughout his youth with his family of five siblings and both parents, with his education and then later as an adult, with his mentors. He constantly emphasized three specific values—loyalty, integrity and honesty - throughout the study, to describe his values as person and leader. To further demonstrate his commitment to these values, Paul offered a list of adages or insightful thoughts that he normally shares with aspiring leaders and current presidents. After asking where he learned these values, he responded:

Paul: My father, our parents. You know, you had to be accountable and respectful, um, and treat everyone with civility. You know, it's kind of like some of the comments I have on there [pointing to a list of sayings or adages he developed over his career].

Researcher: Where do you think that courage comes from, because to me, that takes courage? 
Paul: From our father. Out father always told us that you had to be respectful our family. You know, and protect the name. So, I would say from my father.

And you know, you can't sell that and once you sell that, you're done.

Paul's journey to the presidency and chancellorship had a few instances wherein he admitted he was handpicked for certain leadership positions. For instance, he discussed being handpicked for his first dean's position, or for one presidential position where there was a serious power struggle happening in the institution. However, without these opportunities, he assumed that he may not have had his overall career. He mentioned that during his time as a faculty member, although he had considered leadership at the vice-presidency level, he never desired to be a president. He identified individuals, like the president at Waters College who saw potential in him and, in turn, promoted him to his first position as a dean, without having to apply for the position. However, his life plans were very different in his youth and certain experiences could have prevented him from being who he is today. For example, during Paul's K-12 years, he talked about a time when the school system he grew up in had a marginalizing processes that later affected his dreams of playing football and even finishing high school traditionally. For example, he talked about how his kindergarten was a sorting device for children living in a certain zoning area, particularly in an area with a high percentage of 
second language speakers where he lived. He explained that while other children in other zones only did kindergarten for one year, he did it for two years, although he himself was not a second language speaker. As a result, the following excerpt delineated the ripple effect this school sorting had on him and his dream of having a football career in high school and beyond:

On the west-side of town where all the white kids lived, they [school structure] went from kindergarten to first grade. So then you have to think in terms of age. So you and I start school at age 5, you're white and live on the other side of town. At age 6, you are in the first grade, and at age 6, I am in pre-primary. So, I am a year behind you now but we're the same age. Then I had asthma really bad as a kid and, ah, missed a lot of school, missed a lot of school. In the 5th grade I missed so much school that I flunked the 5th grade--okay. So now, I am two years behind you--okay. So, when I go into high school, I couldn't play sports and my mother wouldn't allow me to play sports because of the asthma, and when I went to high school, I told her I wanted to play. And ah, play football, and she told me that I couldn't play. And I said, "I am in high school now and I should be able to make my own decisions, so we had that conversation." And she said, "Okay you can play, but if you have one asthma attack you goin' quit [sic]--okay." And I had an asthma attack every night. But I didn't say anything... Because back in the olden days when I was young, that was my excuse to get out of everything. 
...Well, in (that state), if you are 19 before September 1, you cannot play intercollegiate---um, inter-athletics at the high school, okay. My birthday is July, so July of that year I turn 19 which meant in my senior year I could not play football. You get the madness of this.

So I dropped out of high school and went to community college because, in (that State), today it's age 18, but back then it was age 19.

Soon after being banned from participating in his high school activities, such as prom because he wasn't an enrolled student, Paul was upset and retaliated by deciding against submitting his transcripts from the community college back to the high school in order to get his high school diploma.

Paul has had a long and successful history in higher education, and, overall, his career spans over 40 years in higher education. He defines himself as servant leader, and defines his leadership agenda as improving the curriculum, through grants and other programs, through capital projects and service, all of which he said he did with the students in mind. His résumé relays the high level of leadership competencies in all areas and functions of the positions he attained, including fundraising, successful million dollar bond measures, managing budgets, and dealing with difficult challenges and crises within the college. In addition, he is the recipient of several honorary mentions and awards.

Being careful at all times to quickly identify the mentors and people who worked alongside him, Paul spoke about a myriad of leaders, board members, 
faculty, staff, students and other individuals who supported him, counseled him, and help him to shape his leadership. Overall, Paul's legacy is full of positive working relationships with all constituency groups throughout his career, which he believes stemmed from his time as a faculty member where he built collegiality and trust and from his many accomplishments to help improve life for all constituency groups at each college for which he worked.

No confidence vote background. Paul was the only participant who received two no confidence votes during his tenure as president and again as chancellor in two different community colleges. Paul's first vote was from a rural college in the northern half of the state (Jago College), and the second was in a central local in the state (Campion College) when he was a chancellor. He had already served as a president at two previous institutions over a six-year span, prior to leading at Jago College, where he got his first vote. Little information is available about this vote due to historical maturation of the data, where meeting minutes were may not have been electronically archived or accessible. Perhaps, this limited data is also has something to do with the actions of Paul, who recalled building close partnerships with all the newspapers and media in the area, as a strategy for protecting the organization. As a matter of fact, this participant was very surprised that I was able to find him and he asked me for details on how I was able to link him with the vote. I explained that I had a break in my research when I 
discovered a news brief involving his somewhat recent candidacy for a chancellorship position, during which time questions about the votes were raised.

Paul reportedly received his first vote in the mid-90s; one reporting agency said the vote happened on date but Paul noted that it happened on another, at Jago College, from the union leaders and not the entire faculty body (Research document, 2). The vote occurred soon after he started his tenure at Jago College; he maintained that it was two years after, was and he opposed that it had happened only a year after his entry in the institution. This was the second vote for Jago College during that time (Research document 3, 2014). Paul acknowledged that the vote was on the "heels of negotiations" and after the college experienced steep budget cuts in the state and the district, this eventually had huge implications on collective bargaining or contract negotiations with the unions. Generally, these votes called for the leader to abdicate his/her position; although the intent of this vote was unclear in Paul's incident. However, Paul mentioned that he had the support of the board, and actually ended his tenure 5-6 years after the vote, in the early 2000s.

With the second vote at Campion College, Paul noted that the vote was a direct reaction to layoff notices that were the result of an egregious clerical error and this happened during a time when he was in the middle of contract negotiations with the faculty as well. Paul joined the institution as a chancellor in 2002 and received the vote in 2003 . He highlighted, and this study confirms, that these layoff 
notices were rescinded, but Paul actions still generated a vote of no confidence on his leadership (Research document, 5). In addition, one newspaper article asserted that Paul's vote was the result of faculty feeling they weren't consulted during these negotiations. Paul further stated that the vote involved a small fraction of the faculty and that the issues and concerns that caused the reaction of the faculty union literally "disappeared" once the layoff notices were rescinded. Again, Paul had the board's support and continued in his tenure there, retiring as Chancellor Emeritus in 2008. Paul now runs his own consulting firm for presidential searches and is involved in general leadership mentoring.

\section{Participant: Bill (pseudonym)}

Personal and professional background. Participant Bill identified himself as a White American and a male, who is also a native in the state where he led as a president in three different community colleges and also served as vice chancellor for his state's system. Bill has been a leader and faculty member in higher education for 37 years, including work in the university setting. Bill's story is rich with the struggles and importance of family throughout his life and balancing his work and family life, so much so that his first presidential job teetered upon whether or not his family was willing to go with him.

Bill attributed his personal development and growth, both as an individual and a leader, to several positive and negative experiences throughout his childhood 
and youth in the small community of Midtown. Bill admits to having a tough home life with both parents and three siblings. His narrative disclosed the effects of this home life on his life choices and he readily admitted that he wasn't the most stellar student and often lacked focus and was distracted by work and friends.

Bill also contrasted a positive role model experience he had with his history teacher with an experience he had with counselor in high school, who apparently dealt with Bill based on what he observed in his behavior, not necessarily seeing his potential. Bill recalled how his history teacher encouraged and challenged him to do well, perhaps because this teacher knew Bill came from disadvantaged circumstances. From Bill's story it is possible that this teacher may have singled him out to support him or that this teacher generally tried to encourage all of his students. Conversely, his high school counselor who was supposed to help encourage and steer him in developing directions for his life, after high school, was condescending and told him "You'll never make it to college. You'll never make it." Compelled to prove this high school counselor wrong, Bill talked about becoming more serious with his school work, pursuing history as a major, completing a doctorate degree and making sure he encouraged and gave hope to students and families as a leader

Bill not only completed high school, but went on to complete a doctorate and his first job in the community college setting was as counselor. Bill's career trajectory has led him through most sectors in education and youth services, 
including his work with non-profit national youth programs, community colleges and universities. Bill's narrative spotlighted key events that propelled him to top leadership positions, and, at times, these events mirrored patterns he had experienced in his youth. For example, during his time at one university, Bill noted that, as a mid-level administrator, the president who was also a mentor, singled him out and noted that he had leadership potential:

I served as a (chief of students) there, ah small community, small college, smallest in the state at the time but we grew rapidly and very successful. I brought some new programs in and a Title III grant and couple of other things. And so within four years, the President said, "You're ready for the presidency." And I said, "I don't think so."

Regardless of Bill's initial protests, this president and mentor essentially afforded him with an opportunity to grow and develop competencies for leading institutions. He has had a long career, with his first presidency in the early 1990s (at Green College) and his last, ending in the 2000s. Bill would still consider his overall leadership style as "eclectic and situational," and he has learned to adapt to styles that each situation demands and has learned to refrain from being rigid. He credits this understanding of being adaptable to his experiences at the Sunshine College where he received a "failed" vote of no confidence. There are moments in his career where he was surprised that he received the president job because he thought he was an unlikely candidate, and other times when he was surprised the college made 
other choices. Overall, he mentioned enjoying his overall career, although he highlighted instances when it was tedious, like during fundraising and eating "rubber chicken dinners" and he is open about his gratitude for the opportunities and his family's support throughout this time. Bill has settled into retirement and is currently working part-time as a teaching faculty in higher education.

No confidence vote background. Bill's vote of no confidence at Sunshine College can be considered highly controversial. According to Bill, the institutional players who raised the motion for a vote "lost the vote" or "it failed" to make it pass. Meaning, the sponsors of the vote enacted the voting process, the outcome was that more individuals voted that they did indeed have confidence in Bill's leadership compared to those who voted that they had lost confidence in him. However, Bill claimed that this vote was an on-going "misunderstanding" that has followed him around, as that several reports have noted that he did indeed receive the vote. For Bill, this "failed" vote was received in 1996, less than one year into his tenure at Sunshine College. He admitted to a few of the rationales and concerns the faculty had about his leadership, such as moving too quickly, but also noted that they hired him as a "change agent" an "outsider" because the institutional players indicated that this was part of the new direction for the college. It was also unclear from his recollection if the board and the faculty had a collective picture of what type of leader they wanted or the type of changes that were needed or appropriate for a new leader and the institution. However, Bill also attributed the vote to tough 
budget times, the closing of a major facility that was a feeder college for students, confusion and concerns over salary raises, and ultimately, the lack of board support from his leadership Bill is the only leader in this study who ended up leaving his/her institution, soon after the resolution was raised. Although he did not credit the "lost vote" for his departure, he did see it as a catalyst in his final decision to leave. He also mentioned the role of the media played in his overall demise as well, and, although the vote count could not be found, these newspaper reports would act as anchors for informing these communities about the vote and that other news cycles simply added to in later reports. Overall, Bill credits his experiences at (Sunshine College) as a key factor in shaping his leadership.

\section{Participant: Debbie (Pseudonym)}

Personal and professional background. Debbie identified herself as a White-American divorcee and single mother of two adult children. Debbie mentioned that she was very proud of her master's in math from a flagship school. Initially, Debbie described her life as simply following her husband's career, trying to raise her children while traveling, finding jobs here and there across the United States and globe, and focusing on her degrees. She too started as a faculty member who had no initial interest in leadership and was quite satisfied in any faculty positions she was able to find to fit her life and family. 
Debbie: And so I taught at... a prep schools... and universities... community colleges, and basically I was — this was before strong-the women's movement. And I was following my husband, uh, you know, we were-his was the family career and so I would find teaching jobs around the edges of his career. When he moved I would start over again and, you know, find a new teaching position in a new place. And eventually, I ended up...teaching full time at (a private university) in (southern end of the state). Compared to other participants, Debbie didn't share as much information about her marriage or raising her daughter. Instead, she focused on her journey to the presidency. Debbie's career started in the early 1980s when she landed a dean's position at a local private university. She noted that she had preferred working in the community college setting and in the classroom. While at this private university, one individual saw her potential and advised her to try looking for a presidency at one community college; however, she would later withdraw her application because she didn't meet the criteria for the job; they wanted someone with more administrative skills. Soon after this episode, she found a leadership position as a dean. While doing her deanery work, Debbie was soon approached by another leader in the system who advised her to apply for what would become her first presidency in the Queens community college setting, where she got the vote. Several years after that, Debbie moved on and held a chancellorship at KC College, 
from which she later retired. Overall, Debbie highlighted the fact that her rise to leadership was not without struggle, particularly as a woman.

Debbie: Well, uh, I'll start by telling you about my final interview with the chancellor. Um... he uh... he had the interview with, uh, with- along with the vice chancellor, also a man. And, uh, I think pur-purposefully was talking a lot about sports and everything, they were going back and forth about sports and, uh, he said—at some point he said something about, uh, "Do you like—are you interested in sports?" And I said, "Well a long time ago I used to be a cheerleader," I said, "Does that count?" You know, and um, then toward the end of the- toward the end - the end of the interview he stood up, he was a rather portly man and he put his, uh, fist on his desk like this, I thought imitating a silverback gorilla, and he looked at me and he said, "Are you a feminist?" And as he said it I thought to myself, "That question is so illegal I must already have this job [laughing]." And so I turned, I looked at him and I smiled and I said, "I was born one."... He just laughed. I'm... I know-I know he thought that was funny. I know by that time he had already decided to hire me. There's no—he's too smart a manhe would never have asked that question if —if he hadn't been. I mean—but he thought-he thought he was being, you know, he thought it was... it was a joke. But um, again $\mathrm{I}-\mathrm{I}^{\prime} l 1$ tell $-\mathrm{I}-\mathrm{I}$ had a number of experiences, now this was after I became president but I would go with, uh, I would go to 
something with maybe one of my male deans or maybe a couple of men and a very—if somebody who didn't know us, they would immediately walk forward, reach out their hand to him and say, " President (last name)." You know, immediately the assumption was that it was the man in the group ... who was president.

In addition, Debbie acknowledged that her leadership has been tested often, but she has stood by her leadership style which she described as being highly collaborative and have shared governance with a good team. She noted that her best strategy has been to help others become leaders as well. Although she explained that she never had an official mentor, she did identify key individuals who were highly supportive of her and her potential to lead. She also singled out an event during her early tenure as president, when she had an open position for a vice president of academic and student services. Debbie highlighted the fact that she was hired in one month, cautioned by an influential faculty member that she should not hire a woman for the position, and that was exactly what she did. Debbie hired an African-American woman, Josie, whom she had met and befriended years prior and made a pact with to help each other, as her second in command person to run the college. Josie would work with Debbie for the greater portion of her presidency at Queens College, with Debbie by her side teaching her how to be a president, and she would later go off to her own presidency a few years later. 
Debbie also highlighted that her "spunkiness" and willingness to go against what others thought she should do was directly related to how she was raised. Her mother was equally spunky and a hard worker, always encouraging her children to do better than she did in life. Her mother's sacrifices to help her children were evident in Debbie's stories and the many lessons she learned from her. Debbie mentioned that she introduced Josie to the wise ways and sayings of her mother, especially when they had a rough day at work:

She was the eldest of - of four living children, in fact. And it was through her working and turning her money over to the family that the younger three all were able to finish high school. Ironically, she was the best student, but, being the eldest, she needed to go to work to supplement the family income as she also was responsible for tutoring her brothers and sisters. Well, and-and of course any big sister helping the younger kids in the family, they weren't always totally cooperative. So she was determined that when she went and had her own family, you know, education was really, really important. In fact, Josie and I used to say, "Ugh, If only we could bottle (Mama)." Mama was her nickname, it was kinda like grandma. We would say if only we could bottle Mama, because if we could take that for kids or even adults who hadn't had that kind of support, educational support. I- I used to say Mama was the educational director, you know, uh, she was the public school. But she- when we were growing up, there were just two of 
us, my brother and me who's younger, she could tell you any day what we were studying and all of our classes. She would drill us on it at home. If she-if we had something that we had to write out to turn in, she would check it and if there was one mistake on it, we would sit there and we would do it over again, you know. And because she had been denied a chance for education, she was gonna make sure her kids, you know, really took advantage of it. So, uh, so we went off to school, you know, on those early grades being-every teacher's pet, obviously. And you know that forms your self-concept and then you carry it on. You know, you do that right at the beginning, that's why I so believe in these early education programs because you start out with that sense of what you can do and, I mean, she helped high expectations for us but also supported us in it. And uh, and it just, and we were talking earlier, self-suffi-she wanted to make sure, you know, we were both self-sufficient. She insisted I take typing and shorthand in high school in addition to everything else because you never knew when my future husband might become ill or something. And I might have to work to support the family. And she did not want me selling lipsticks in a local farmer store.

Debbie: But typing and short-hand would get me a decent job, I could least, I could be self-sufficient and it wouldn't fear to support a family if I had to. You know, she didn't think too big.... 
Overall, Debbie appeared to have enjoyed her time in higher education and is quite proud of her accomplishments. She often became animated about her work with institutional players, the diversity of mindsets she came across, the people she and others helped along the way to build their careers and programs, and the community of people she served while in leadership. Debbie, now retired, has not mentioned any real interested in returning to the industry at this time and is happily focused on her family and her artistic craft.

No confidence vote background. Debbie's vote of no confidence came in the mid-1990s, only four years after she had been a president at Queens College. This was the first no confidence vote in the history of that institution, but it wasn't to be the last. According to Debbie and the state agency that collected information about these votes, the vote came amidst strained relationships with the faculty over shared governance concerns. More specifically, the faculty was accusing Debbie of a lack of consultation with them, particularly in areas of staff restructuring and hiring during times of serious budget cuts. Debbie identified a small handful of the faculty who were being encouraged by "the cosmic accidents," a group of faculty who regarded themselves as the founding faculty and leaders in the institution and who wanted to have a key role in the leadership of the institution, but Debbie admitted she did not make space for them. Debbie suggested that this group was instrumental in inciting a possible 60 other faculty members to give her a vote 
against her leadership. Debbie admitted that state mandates had required budget cuts, and that she was indeed reorganizing the college due to fiscal cuts but she stated that she did not agree with the faculty on which positions to cut or hire, faculty vs administrator. As a result, Debbie advised that she saw the vote coming because, although it was a tenuous time in the institution, the use of a no confidence vote was a new wave sweeping over the colleges at that time. She noted that although she thought it might happen "it was too late" to do anything or try to stop it. Essentially, it was unclear what the faculty wanted to happen, a sentiment noted in a WASC self-study done by the institution, but she would stay on for several years because she had strong support from the board and district chancellor. They incorporated several changes, including increased communication with institutional players and space for the faculty who wanted some leadership role in the college by expanding college council, a group she refers to as a "Noah's Ark...two of [everything]." Overall, Debbie noted that although she did not leave the institution after the vote or because of the vote, a reaction she believes the voting faculty were probably looking for, she did admit that her sentiments about her working relationships with the faculty were not only strained from that point on but she had shifted in the way she thought about them. 


\section{Participant: Susan (pseudonym)}

Personal and professional background. Susan identified herself as a

White married woman and mother of one daughter, who retired in her late 50 s to play the role of grandmother for her grandchildren, after working in higher education for more than $30+$ years. Susan spoke about the importance of her family and particularly about her husband who was also a faculty in the institutions where she worked and who is now retired. Susan also shared a cursory explanation about marrying her husband, a faculty at time, when she was only 18 years old.

Susan constructed her identity by focusing on her upbringing and highlighted her trajectory to leadership positions. She noted that, while growing up, her lens about life and then leadership was shaped by her family, involvement in a debate team, and her involvement in church or religion. For example, when asked where she got her insights about life and her values, Susan talked about learning to search and research information for herself because of her involvement on debate teams in school and from her family, particularly her mother who she said taught her to "always do the right things." As a result, she became more observant about the world around her and at one point questioned some of the thinking of her conservative family about social issues. This did not always go over well with her family; at one point, she mentioned that family members referred to her growth and lens as "going downhill": 
Susan: .... I still remember that. I was like 15/ 16 years old. And I remember telling my mom that was kind of a start of something for me, because I remember saying to her, "I can't believe that because (Jessica) is Jewish, that she is going to hell." Somehow, I am sorry, but that just doesn't work for me. So, to answer your question, yes, components of my spiritual background definitely affected that. And then my mother, which I am far more liberal than my mom, but she has come leaps and bounds in the last 30 years and I still blamed her. I'd say, "You taught me to do the right thing, you just didn't realize this was what it was going to be like. This is why you don't use this type of language; this is why you champion this." You know, the big issue for us, and she is coming a long way, she is not quite there.

In another vignette about her personal development and how her life was influenced by family and religion, Susan mentioned her growing concerns about race and racial issues around her and nationally, and how she tackled those issues within her immediate family at the time. For example, Susan describes an episode where she had to deal with the intersection of race and the family in this way:

Susan: Now I would say this, I was brought up in a very conservative, (traditional protestant) home. I do not practice that anymore, they are way too conservative for me. And on the tolerance part in particular, and I have talked to my mother---my mother and I are very close, and I've talked to my mother about this and I said, "You know mom, when I used to go to Sunday 
school, I'd win all the contests for memorizing, you know, the most Bible verses and that stuff...." I said, "You know we sang Jesus loves all the children of the world: red, and yellow, black and white right..." And I said, "You know what, I thought you all meant it!" I said, "I thought the church..."

Researcher: How old were you?

Susan: When I told her this? Oh, when I told her this [pause] I was an adult, when I was an adult.

Researcher: Okay [chuckled].

Susan: But we were talking about how the arguments that I had with my grandma, who I loved, but during the days of Martin Luther King Jr. and they thought he was awful. And I would say, "What are you talking about?" It was like as an adult, and my parents would say it all the time, that high school speech corrupted me. I was in competitive speech and debate... Susan: So, I learned how to do research, I learned how to do arguments, and of course, I learned how to think, critically. So they said that's when we started, you know, going downhill. I mean they loved me, but anyway. But I still go back and I tell her today, I said, " I—even though I don't practice the dogma of the faith I was brought up in, I still practice the principles, that I was taught. I happen to have learned that other religions teach some of those 
same principles and that we didn't have the corner on the market, that I thought we did.

Overall, Susan explained that her upbringing also influenced the ways in which she thought about work. She mentioned how her upbringing not only bled over unto her career and career path, but it was heavily supported by her husband and modestly by constituents who served under her leadership. This is also evident in her leadership lens and practice which strongly focused on increasing consciousness around diversity and inclusion and being intentional in creating opportunities for individuals who don't always have access to certain spaces.

Susan's path to the presidency is a unique one by today's standards. She mentioned that she was a "home grown" president, meaning she became a leader over the institution where she had also taught as a faculty. Susan described her path as atypical today and suggested that there might be some drawbacks to leading an organization when you are "internal." For instance, Susan described her career path as follows:

Susan: Well, I started as a community college alum, that's always a part of my story and I think that's important. So I'm graduate of (a community college in this state). I, um, started my degrees in (the social sciences and counseling). Then I started my career as an (teacher), so I taught 4th grade for 3 years, stepped out to have a baby, got my master's and as I started to work on my doctorate, I started teaching as an adjunct at the community 
college. I married a community college professor when I was, uh, a student. So that's quite another story. So I was really tied to the community college already. So, I, uh, was an adjunct professor for six years, while I also got my doctorate. While I also taught at the (a university) that was nearby, so I was doing all three. And then became a full time faculty member. A couple years later became an (academic senate leader-pseudonym) and also the executive board of the faculty union.

Susan: And then, just a year or two after that, as we say, crossed over to the other side, I became an administrator. [I] started a new program that didn't exist in (a consulting and training services to employers and their employees in the community). So I went from the Liberal Arts, Social Sciences to a vocational... [interrupted by knocking at the door].

Susan: [continues] I crossed over and helped start a brand new program (involving different industries and sectors) and the significance of that was all my work had been in student services and Liberal Arts instruction and now I was the vocational technical areas which was a very good thing, uh, in terms of broadening my horizon. I was also- became the director of special programs, wrote lots of grants, uh, and then became a district administrator, ah, as a vice-I was called an assistant chancellor, doing 
some of those things actually. Basically, when I also became an administrator, everything I did stayed with me.

Susan: Got a new position, kept the old one. Got another-another new position, kept the previous two... Um, so career wise-and then I became Chancellor in that district. So I am home grown.

In addition, Susan noted that she was one of the first six women presidents and chancellors at that time in that state, which has been documented in other reports in that system (Agency document, 11). She also mentioned that she was proud of her 30 years of experience in higher education, with more than 12 years at Wolmer's College as faculty and administrator and then another 18 years in Hugh College District as vice chancellor and then chancellor. She noted that she had transitioned from teacher to faculty and then to administrator, all the while learning and developing and preparing to take on the role as Chancellor, with some mentoring from her predecessor and former chancellor. She explained that constituents would probably describe her style as down to earth, and she mentions that even one person said to her:

Wow, you-you are really don't talk like a mucky-muck." And it took me by surprise and I said, "Excuse me, what do you mean?" And I knew what she meant. So the down to earth, I think that works for me. And then, a nice compliment that I have gotten from a lot of people, although I don't think of myself as this way, was that they would say that, "You've got courage, you 
are very courageous." And I heard that a lot, particularly during the (equity and diversity) initiative days and then this last summer [at a nearby college]. And I-so I guess that's part of my style, but I still think of it as just doing the right thing...

Furthermore, Susan noted that her leadership style and practices were based on building relationships with others. More specifically, she described her style and practice as more akin to the Emotional Intelligence (EI) literature (Goleman, 1998), described as understanding one's self and practice through one's own self and social awareness and management of self. In detailing out her leadership style and practice, Susan noted:

Susan: Yeah, I teach it all the time and I believe in it. When Emotional Intelligence first started getting popular about 10 years ago and I read Goleman's first book. I went, "Yes, yes, yes, this is it, this is what I do, I knew this is why this works." But I didn't have the research to prove it. I wish I had written the book. So, I don't know how familiar you are with it, but I absolutely believe in it. So some of the concepts are 1) competency. To me competency is the basic, you have to have the competencies; you can't fake it. Okay, so that's just a given, and you have to be half way intelligent to keep up with stuff. So then what do you have to do? [2)] Mostly, I think you have to be authentic, I think you have to be caring and compassionate, and I think you have to know how to communicate. Using your earlier 
question, which includes listening a lot, letting people know you care for them, being a real person, um, being the thing you asked about before, being collaborative, trying to build consensus. All the stuff people want from you that everybody wants from everybody, far as I am concerned, that's what everybody wants, and then at the same time, being willing to make a decision. Gather the information, do your homework, be collaborative and then make a decision and not shy away from the tough decisions. As a woman in leadership, I think some of us were maybe too reluctant to make the tough decisions. Maybe, I don't know for sure, I am not close enough to see what everybody did, every day. No, I take it back. I have known some who didn't, 'cause I have sit on too many accreditation teams. But some men too, it wasn't always exclusively women.

Another final important highlight about Susan and her leadership was that, although she was successful in her leadership, there were some drawbacks and even resistance to her leadership, which she inferred has to do with being a leader from the inside. Susan admitted that being homegrown didn't mean that everyone accepted her as a leader and that gender was also a key factor in how they responded to her leadership. For example, she mentions that gender played a major role in this way:

[Speaking about gender]...And I think it matters directly to your topic, that when I was strong on a topic, or an issue, or an action, of course, I was seen 
as, you know, bitchy and, you know, aggressive, and all that other stuff. Even though, the very people who went after me with the vote of no confidence had really gone after my predecessor on a number of issues, the male, the white male. [They] thought he was stubborn, wouldn't listen them, didn't understand education, all kinds of nasty, ugly stuff. But, nobody ever thought about doing a vote of no confidence. They were thethat same group, I know I am getting ahead of myself....

Susan: But that same group was actually happy when I became chancellor because I was one of them. Remember, I was one of their own...

Researcher: One of their own [overlapping talk].

Susan: I was home grown, I had ten years in the classroom, he had none [referring to her predecessor]. You know, I've been on their side on negotiations and even my six years as vice chancellor, I was often the mediator between them and him.

Susan: But my favorite story about does if it matter if you were a women: I had two different administrators, male administrators, who I was confronting about their behavior say to me, and this is a direct quote, "No damn woman is going to tell me what to do." And I was chancellor! Researcher: Interesting....

Susan: And I responded immediately to the first one and deliberately to the second one and said, "Have you noticed lately the name at the bottom of 
your check." But anyway, neither one of those people are still around or stayed around too much longer. So, yeah, it mattered all the time.

Overall, Susan described how proud she was of her work as a faculty and chancellor, and in her efforts to help create a model for shared governance for that district. She mentioned that she was still very connected to the state's system and does an exceptional amount of consulting and seminars to assist other leaders, boards and agencies with no confidence votes. Susan appeared to have enjoyed her time in community colleges and in leadership, and openly shared lessons she learned from all her experiences.

No confidence vote background. Susan's vote of no confidence also occurred in the mid-1990s and it was not only a vote against her leadership but Susan noted that it was also a vote against the board as well. This information was not apparent in newspapers articles or in other reports about her vote. These documents focused of the faculty who gave the vote who placed the target of the vote squarely on Susan, and that their desire was for her to leave. In addition, in all the reporting of the vote, it was also unclear how many faculty members actually voted against her leadership.

Susan inferred that the vote emerged out of a perfect storm, a storm precipitated by three major events. The things she highlighted were, first, while focusing on increasing diversity and inclusion, she hired a Latina President who, 
although was one of three on the short list of faculty, was not ideal choice of the faculty. Second, some of the faculty were upset with her $13 \%$ raise during a crucial time of budget cuts (Document 11, 1997). She noted that this was a raise she has been promised by the Board, and she remembers telling the Board to give her a 4 or $5 \%$ raise and they did not agree. The board was outraged by the faculty's response but went ahead and gave her the raise. Third, Susan mentioned that the tipping point came when she followed through on firing a campus president, who had made some unethical business deals during his tenure. She had initially given this president a year to find another job prior to the day she appeared with a board member to dismiss him. She noted that that the firing actually happened simultaneously with the vote, and she and the board member asked to meet with the academic senate during their meeting to come up with the vote, but they refused to speak with either of them. The Bill of Particulars from the faculty in Susan's agency also mentioned that they gave her the vote as a result of her "abuse of power" and her "endangering the sovereignty (of the college). Susan clarified this accusation, noting that:

There was no abuse of power it's just that they had a strong leader who would not let them be abusive of others. In fact, one of the compromises that we made, because it was a power struggle, is that - that we made is, um, was that after that point we allowed the faculty to have one representative they would elect between the two, the senate and the union, to sit with the board. And that person would take on the role of faculty-liaison. Because my board 
was a good board, that's very critical to my success. They were a strong board, they practiced good trusteeship and they weren't going to be pushed around. They were devastated by this [referring to the board].

Subsequently, once the shock wave of the vote had occurred, Susan asserted that she and the board worked hard to actively address the faculty's concerns of the faculty by having a succession of meetings to come up with a plan to provide them with space to share in the leadership of certain things. Finally, Susan's reaction to the vote was unique, when compared to those of the other participant, in two ways: (a) Susan received a vote from her fellow colleagues she had worked with throughout her career, and (b) she was the only participant to address the vote directly in newspaper reports, soon after the vote was given, telling the media she was not planning to leave (see Document 13). Susan explained that she could have only done that because she had the complete support of the board and that of her husband, who was a former colleague of these other faculty members.

In summary, these leaders constructed and shared very rich and in-depth narratives about their lives and experiences as a CEO, narratives that only they can tell. As noted in Chapter Three, the process of interviewing such elite participants can be one of the most tenuous processes of any study, a concept noted in literature (Bogdan \& Biklen, 2007; Hochschild, 2009). According to Hochschild (2009), unlike a survey process, an in-depth interview requires perhaps more preparation about the content being studied and transparent strictness in maintaining confidence 
and meeting all the ethical considerations of the study. For any given reason, such participants can be one of the most challenging groups to interview because it might be difficult to build enough trust during the process or enough to help the leader disclose their stories in as uncensored a manner as possible.

To this researcher's surprise, these leaders were able to share things with this study that they mentioned they had never really thought about or shared before. Three participants did explain that they have talked about the vote before in proactively ways as something leaders can survive, and they shared their stories in seminars, or during job search processes. Perhaps, at that time and while they were still working in their institutions, they weren't able to be as personal, reflexive or open about the matter in previous venues, and, perhaps, they were able to do so now because they were retired and not necessarily working in same capacity in CCC system. There were several moments when I was asked to leave out certain comments or incident from this process. Overall, it this process was extremely exhilarating and an honor to have had these conversations with these presidents who were able to openly share their stories with me and lend their time to this study. The focus of the next section of findings will uncover the themes that emerged from these stories with a focus on the areas were these themes converged, diverged and left gaps. 


\section{Thematic Findings and Results}

With the aforementioned research questions in mind, this analytical process began with an examination and deconstruction of these four rich-narratives or counter-narratives (Delgado, 1989) of these four participants-Bill, Debbie, Paul and Susan-into four significant themes of the study. Each participant provided a full narrative of the vote on his/her leadership, which amounted to a total of five votes, with one participant receiving two votes from two different institutions. As mentioned in Chapter Three, these themes have also been developed using a detailed analysis of these in-depth interviews and has been triangulated with other research documents and data memos; a quintessential process for exploring the rich descriptive data with its many diverging points and contradictions (Bogdan \& Biklen, 2007).

During the first round of thematic analysis, a cyclical process for finding and organizing the most salient themes (Bogdan \& Biklen, 2007), four major themes emerged with a corresponding 44 subthemes in total, see Figure 10 below. The four major themes of this study are (a) known triggers of NCVs, (b) crucial blind spots, (c) implications of the vote on leadership, and (d) lessons learned about the vote. However, by the second and final round of thematic analysis, these subthemes were again synthesized comfortably into six subthemes, see Table 9 below. All these themes and subthemes were central in this study's inductive and interpretative processes, guided by the fundamental question in the thematic 
analysis process: "What is the story?" (Weick et al., 2005, p. 314). This question was crucial in the researcher's process for interpreting, framing and trying to make sense of what happened to each CEO participant. The key objective of this section was to move beyond the glaring issues already highlighted in the previous section, to reframe the causes and extrapolate other stimuli in these situations that these leaders may or may have noticed but talked about in their stories. Following this section, the chapter will conclude with a brief overview of this chapter's findings.

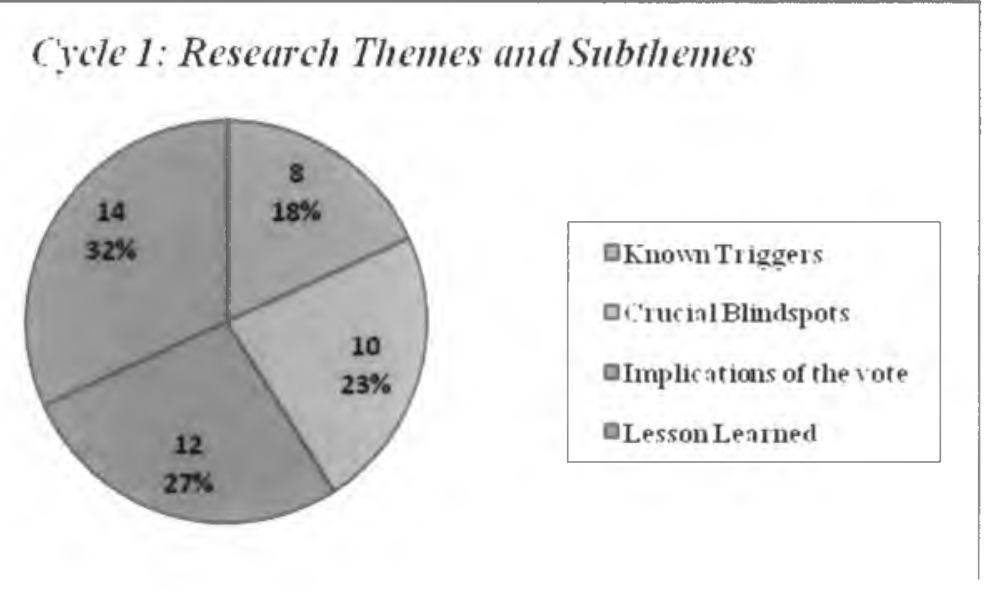

Figure 10: Data Research Themes and Subthemes chart illustrates the highlighted four main themes with the number its corresponding subthemes, which emerged from the data most coded and referenced. 
Table 13.

Cycle 2: Research Themes and Subthemes

\begin{tabular}{|l|l|}
\hline \multicolumn{2}{|c|}{ Research Themes and Subthemes } \\
\hline Major Themes & Subthemes \\
\hline 1. Known Triggers of NCVs & 1. Institutional Triggers \\
\cline { 2 - 3 } & 2. Leadership Triggers \\
\hline 2. Crucial Blind Spots in NCVs & 3. Hidden Social Forces \\
\hline 3. Implications of NCVs & 4. Personal Implications \\
\hline & \\
\cline { 2 - 3 } & 5. Professional Implications \\
\hline
\end{tabular}

Note. This table presents a breakdown of the themes and subthemes that emerged during data and theme analysis. Note: The concept no confidence vote has been denoted as NCV and will be used throughout the rest of this chapter.

\section{Theme One: Known Triggers of No Confidence Votes (NCV)}

The term triggers was selected because it summed up the idea of "something causing something else to happen" (Merriam-Webster, 2015. Subsequently, a known trigger is being described here as the something causing NCVs to happen, otherwise known as an attributable factor. Each participant reported several triggers that he or she was aware of, as they described different contextual factors, such as years of strained budgets, contract negotiations gone 
awry, leadership changes and demands. Once all the themes were synthesized, two major subthemes encapsulated the triggers these leaders framed as attributable factors in their stories: (a) institutional triggers and (2) leadership triggers.

Institutional triggers. The theme of institutional triggers refers to the attributable factors in each participant's institutional contexts, such as something within the district or the state system that participants believed contributed to the vote. These were the areas within the institutional context that leaders knew might pose a problem for them in one way or another. Collectively, all participants identified and selected two major institutional triggers that they believed contributed to vote in their stories: (a) structural reforms and laws such as AB 1725 that focused on institutional governance, and (b) on-going budget constraints and budget cuts in the system that these presidents had to be responsive to.

Structural reforms. The first institutional trigger, a new structural reform or law known as AB1725 at that time, was a known and directly attributable factor for two of the four participants. The AB1725 law was enacted in 1988 to increase the participation of faculty, staff and students in governance over specific areas in community colleges, as noted in the Education Code Section 70901(b) (1) (E), required administrators to strengthen the role of the local academic senates by consulting collegially with them on such as issues as hiring (Gabriner, 1995), as discussed in Chapter Two. Participants discussed how this law, also known as the California Community College Reform Act and more popularized by the term 
shared governance, not only changed the structure of decision-making in their institutions, and in some cases slowed it down, but increased confusion about how governance would work on their respective campuses for all constituents. Essentially, the law specified 10 areas where faculty should be consulted before presidents or chancellors made a decision (Education Code Section 70901(b) (1) (E)). As noted in Chapter Two, these areas for consultation included, curriculum, grading, college governance structures, processes for institutional planning and budget development, to name a few (Education Code Section 70901(b)(1)(E)). In addition, the law added one additional area—other academic and professional matters mutually agreed upon-which others believed was the key area that generated different interpretations and challenges between faculty and administrators across community colleges (Collins, 2002; State Academic Senate, 2014). Essentially, the AB1725 law and governance structures at the time did not provide a clear model of how this law would or could work, and there were growing misinterpretations about what it would mean for college campuses and leaders overseeing these campuses. Consequently, this law posed a challenge for most institutional players, and two of the four participants in this study experienced the challenges of shared governance as faculty and as an administrator.

Debbie's narrative offered the fullest picture of these misunderstandings in shared governance. The following two excerpts began soon after AB 1725 had passed and Debbie was a president at (Queen's College) and she talks about what 
she observed in the mindsets, attitudes and understandings the faculty had in interpreting the new law:

Excerpt 1: I don't remember if I told you this last time but ah.... We hadwe had had to - the decision about faculty and how many faculty we had to hire or what percentage you're gonna have of part-timers or whatever, was made at the district office. Uh, that was not something that, you know, that I had control over. But the attitude, not from the faculty of the whole district, but from the faculty at (Queens College-pseudonym) was [using a husky tone to represent the faculty's voice], "Well, if we have to cut faculty, then we have to cut, you know, everything else." Well, you know, that's a little different because if we had to cut faculty, you still have a part-timer teaching the class. But, if you cut a classified position, you don't a parttimer taking over that job. You're doing it with one less person. And same with an administrative staff, if you cut an administrator, then that's one less person doing the job. It's not that I like having more part-timers.... So nobody—it's not like anybody is sitting there going, "Yeah, hire more parttimers, we want more part-timers [participants hands in the air in a fist like position]." But, I mean, that was—-that was the reaction of the faculty of cut, cut, cut, cut, you know.

Excerpt 2: I remember [chuckling] Josie and I had this—-we did a retreat with, uh, with some of the faculty leaders and one of them, she was a 
woman who was very involved in the union, and she said, "Well this is gonna make your job so much easier, because we're gonna-we're gonna take on some of that responsibility for you." And you know [chuckling], Josie and I were looking at it, at each other like, "Oh yeah, boy does she not understand what this is all about [laughing]." Oh our jobs are gonna be so much easier now [laughing sarcastically]. We'll only have ten committees to keep track of. Um, so that was one piece of it.

In Debbie's narratives, she also mentioned that the faculty who gave her the vote cited a lack of consultation regarding hiring and personnel cuts that were also noted in other newspaper documents (Document 3). In response to the vote and this known trigger surrounding shared governance, Debbie noted that she could see the vote coming in the following excerpt:

Debbie: Well, it was uh, there were-you know, it's very interesting, because I could see it coming, you know, sort of like seeing it, you know. But I really was, was not in a position to be able to- to shift the course. Uh, part of what, uh, contributed to it was just the whole $\mathrm{AB} 1725$ thing. However, when $A B 1725$ passed, the reaction in general from-from faculty, and this is all, an over-simplification that it was sort of like, "Oh, now shared governance. So everybody takes ten giant steps forward." Well, quite frankly, the way, I mean, I was involved-I was on that CEO board when $\mathrm{AB} 1725$ was being formulated. We were already doing what-what 
was the intent of $\mathrm{AB}$ 1725. We were already doing it. But that's not the way it was seen by particularly those who were-who were, you know, very active and, and those more likely to be in the union. And they saw this, okay, now it's, you know, major step forward. So it didn't matter whether you were - whether you were already there or not, everybody expected you to take ten giant steps forward. So that was part of it [pause] that expectthe expectation that was involved with - with AB $1725 \ldots$

Debbie: Yeah, I could see it; I could see these things converging... At any rate, so I insisted-oh, I know what I was going to say-the other thing is, given the total number of faculty, um, it is not uncommon for the vocational programs to suffer, if it is turned over to faculty to determine priority. Because there does get to be a little bit of I'll scratch your back if you scratch my back in there. And so, you got, you know, how many math faculty do you have and how many English faculty do you have? And you always have tremendous enrollment needs in those areas. So are they going to be sensitive to a vocational program that, you know, only has 2 or 3 faculty members and really desperately needs another one to keep that program balanced. They, yes, they need another one because they got so many part-timers in there now but in terms of the program needs, you can get some good-good part-timers teaching ESL and composition whereas you can't get part-timers teaching this Auto Tech program or whatever the 
thing is, you know. So again, that's the kind of-those are the reasons why that was an area that I.... So that was one area that some colleges totally gave over to faculty and I did not. Now, I don't know how it's worked out since - since it shifted at Queens College, but uh, I do know that those people who were vice presidents there, during the time I was there, primarily in the instructional area, I mean, less so in the student services area, but those vice presidents strongly agreed that it was important too. Debbie: So—ah, so that's one—as I say, that was one piece where I did not allow everything to be done by a shared governance mode. And I would support that to this day.... So that was another case for them to say, "You see it, she is not practicing shared governance, she's not letting us decide which administrative positions to cut... And I would say that to this day, I mean that is not something you turn over to shared governance [chuckles].... You're responsible for running the institution and you need to- to determine what kind of configuration of support administrative staff you need.

Another participant, Susan, had been accused by the faculty of abusing her power in her hiring practices and consulting with the faculty; she was also told that her actions posed a danger to the sovereignty of college. Susan mentioned that both of the institutions she oversaw in that district were already highly collaborative and doing most of what shared governance was asking for, but the mindsets of the 
faculty had shifted. The following excerpt delves into an occasion she identifies as the place where institutional players were saying she was abusing her power and not consulting with them during a presidential hiring process:

Susan: We also had another rule in our document that said, "If there was a time urgency, then I would tell them that."' And say, "Look, we have to agree on our five-year priorities for the (funding plan), cause if we don't turn it in to the Chancellor's Office by (a certain date), we will be off the list." So we don't get to talk about this one for six months. So that stuff helped.

Susan: Um, it caused a problem, partly because of the diversity thing, I confess, but also because [pause] a group of powerful men, primarily on campus, did not understand the process that we were doing to three finalists, it would be my and the board's decision, not theirs.

Susan: And they thought that right down to the end, I should consult with them and have them, you know, decide the final one and that wasn't the process, it wasn't what we were doing, so you know, it's—it's—it's questionable how much the diversity had to do it do with it. I think it had more to do with who was going to make the final decision, the board and the chancellor, or this group of faculty. 